\title{
ESTÍMULOS VERBALES Y TRASTORNOS EMOCIONALES: UN ESTUDIO SOBRE PALABRAS CON CONTENIDO EMOCIONAL
}

\author{
M.Teresa Blanch y Rosa M.Baños ${ }^{2}$ \\ 'Unidad de Salud Mental de Altabix-Elx (Alicante) \\ y 2 Departamento de Personalidad, Evaluación y Tratamiento Psicológicos, \\ Universitat de València.
}

\begin{abstract}
RESUMEN
Este trabajo tiene como objetivo fundamental el proporcionar datos normativos sobre un listado de palabras que pueda ser utilizado por los investigadores interesados en el estudio cognitivo de los trastornos emocionales. Se analizan datos relativos a la frecuencia subjetiva de uso de las palabras que normalmente se usan en estos estudios, en qué medida estas palabras se relacionan con ansiedad y con depresión, hasta qué punto son comprendidas por los sujetos, y en qué grado pueden evocar una imagen. Además, se ofrecen resultados sobre las diferencias en estas categorias en función del sexo, ser o no psicoólogo/a, la edad y el nivel educativo.

Creemos que este trabajo puede ser de cierta utilidad para los investigadores interesados en el estudio experimental de los factores cognitivos involucrados en los trastornos emocionales, aportándo información que a veces no es fácil obtener, pero que supone un paso preliminar para seleccionar el material estimular necesario en diversos paradigmas experimentales.
\end{abstract}

PALABRAS CLAVE: frecuencia de uso, familiaridad, trastornos emocionales, sesgos cognitivos, palabras emocionales.

\section{ABSTRACT}

The purpose of this paper is to provide normative data of emotional words that can be used by researchers interested in cognitive aspects of emotional disorders. We provide data on word frequency, comprehension, imaginability and the degree to which these words are related to anxiety and depression. Besides, we analysed differences on these variables with respect to sex, age, cultural level and lay/expert in psychology.

We hope this study could be useful for researchers interested in experimental investigation of cognitive bias in emotional disorders. This paper provides information no easily available in our language, which is relevant to be used as verbal stimuli in cognitive-emotional experimental tasks.

KEY WORDS: word frequency, familiarity, emotional disorders, cognitive biases, emotional words.

Correspondencia: Rosa M. Banos. Departamento de Personalidad, Evaluación y Tratamientos Psicológicos. Avda. Blasco Ibanez, 21, 46010-Valencia. Tfno: (96) 3864476 . E-Mail: banos@uv.es 


\section{INTRODUCCIÓN}

El estudio de la ansiedad y la depresión ha interesado siempre a los psicólogos y psiquiatras, quienes para explicar estos fenómenos han utilizado mecanismos muy diversos. Desde hace ya varios años, uno de los acercamientos psicológicos que más atención está recibiendo por parte de los investigadores es el que parte de la teoría del procesamiento de la información (MacLeod y Mathews, 1991). Son muchos los autores y teorias que existen dentro de esta perspectiva. En general, podríamos decir que todos ellos compartirian el supuesto básico de que los trastornos emocionales están causados o mantenidos por distorsiones y/o sesgos cognitivos (Eysenck, 1992). Con el fin de comprobar las distintas hipótesis sobre el papel de los factores cognitivos en estos trastornos, se han utilizado diversas perspectivas metodológicas. Asi, muchos de los estudios realizados han utilizado fundamentalmente técnicas de auto-informe con el fin de investigar creencias, expectativas, actitudes, etc., pudiéndose encuadrar dentro de lo que Brewin (1988) y Belloch e lbáñez (1991) denominan psicología cognitiva social y de la personalidad. Sin embargo, otros estudios se han decantado por la utilización de tareas de laboratorio para investigar procesos, operaciones y estructuras cognitivas, encuadrándose en este caso dentro la perspectiva denominada psicologia cognitiva experimental (Belloch e lbáñez, 1991; Brewin, 1988). Dentro de este último enfoque, se han aplicado diversas tareas experimentales, como la escucha dicótica, la versión modificada del paradigma Stroop, la tarea de detección de puntos, la decisión léxica, palabras homófonas, tareas de autoadscripción de rasgos, tareas de memoria implicita, etc.

La mayoria de estas tareas experimentales utilizan palabras de diverso contenido emocional como material estimular. Una cuestión importante es cómo seleccionar estas palabras, ya que tendrian que cumplir una serie de requisitos metodológicos a la par que poseer significación o valencia emocional relevante para el trastorno objeto de estudio. Para atender a la significación o valencia emocional se suelen utilizar palabras extraídas de cuestionarios, de estudios sobre emociones, etc., que luego son valoradas siguiendo dos estrategias diferentes. La primera de ellas consiste en utilizar amplias muestras de sujetos (generalmente estudiantes universitarios o pacientes clínicos), a los que se pide que examinen las palabras en función de su valor autodescriptivo (p.ej., Gotlib y Cane, 1987; Greenberg, Vázquez y Alloy 1988; Sanz, 1994). La segunda estrategia consiste en utilizar el criterio de varios jueces expertos para clasificar las palabras, atendiendo a distintas categorías (p.ej., relacionadas con amenaza social, con amenaza física, con depresión, etc.) (p.ej., Mathews y Klug, 1993; Mogg. Bradley, Williams y Mathews, 1993). Sin embargo, existen pocos estudios que realicen este trabajo; lo más habitual es que los autores tomen las palabras de otros articulos ya publicados. Asi, por ejemplo, en el caso de nuestro idioma, normalmente se han traducido las palabras utilizadas en otros trabajos anglosajones. Esta estrategia puede acarrear ciertas amenazas a la validez de los resultados. Por ejemplo, puede que existan diferencias culturales entre las poblaciones de habla inglesa y habla española en cuanto a los términos concretos que utilizan para denominar rasgos de personalidad (Sanz, 1994), estados de ánimo, o situaciones y objetos emocionales.

En cuanto a los requisitos metodológicos, las palabras normalmente se han de balancear teniendo en cuenta el número de letras, el número de sílabas, la frecuencia de uso, la imaginabilidad, la familiaridad, el número de significados alternativos, etc. Sin embargo, normalmente se atiende exclusivamente a la longitud de las palabras y a la frecuencia de uso. Para cumplir el requisito de la frecuencia de uso existen algunos diccionarios de palabras inglesas diseñados para tal fin, siendo los más usados los de Carroll, Davies y Richman (1971), Kucera y Francis (1967), y Roget (1962). Sin embargo, en el caso del idioma castellano, sólo contamos con el diccionario de Juilland y Chang Rodriguez (1964) y, desgraciadamente, este trabajo cuenta con algunos inconvenientes. Primero, la necesidad de una actualización de la base de datos, ya que muy probablemente el uso de las palabras haya variado en estos últimos 30 años. Segundo, y con respecto al estudio de los trastornos emocionales, este diccionario no contiene información sobre las palabras 
relevantes en el estudio de estos trastornos. Esta limitación tiene repercusiones importantes a la hora de realizar investigaciones sobre este tópico. Por ejemplo, en un estudio sobre auto-esquemas depresivos y ansiosos, Sanz (1994) no pudo controlar la frecuencia de uso de los adjetivos que utilizaba, porque en este diccionario sólo se encontraba el $39 \%$ de los estímulos que necesitaba. Esto mismo nos ha ocurrido también a nosotras cuando, al diseñar estudios sobre sesgos cognitivos en trastornos de ansiedad y depresión, nos hemos encontrado con la imposibilidad de conocer la frecuencia de uso de aquellas palabras que los jueces expertos señalaban como relevantes para estos pacientes.

Pero, además de la frecuencia de uso, las tareas experimentales suelen exigir que se controlen otras variables como la emocionabilidad, la imaginabilidad o la comprensibilidad de una palabra. La literatura al respecto señala que estas variables pueden afectar de manera importante a la realización de las tareas (p.ej., Campos y González, 1991; Graves, Landis y Goodglass, 1981; MacLeod, 1991, Martin, Williams y Clark, 1991; Paivio, 1971; Pascual, 1984). Sin embargo, como señala Sanz (1994), la mayoría de las investigaciones en español sobre trastornos emocionales que utilizan material verbal, suelen tener como deficiencia el pobre o nulo control sobre estas características de las palabras. $Y$ esto podría afectar de un modo importante a la validez de los resultados obtenidos en estos estudios, ya que estas características de los estimulos verbales podrían actuar como variables extrañas que, al no ser controladas, oscurecerían la interpretación de los resultados.

Precisamente, el objetivo fundamental del presente trabajo es el de proporcionar datos normativos sobre un listado de palabras que pueda ser utilizado por los investigadores interesados en la psicopatologia de los trastornos emocionales. Para ello, hemos analizando cuál es la frecuencia subjetiva de uso de las palabras que normalmente se usan en estos estudios, en qué medida estas palabras se relacionan con ansiedad y con depresión, hasta qué punto son comprendidas por los sujetos, y en qué grado pueden evocar una imagen. Por último, estamos interesados en saber si existen diferencias en estas catego- rías en función del sexo, entre los psicólogos y los no psicólogos, entre los diferentes grupos de edad y entre los distintos niveles de estudios.

\section{METODO \\ Sujetos}

La muestra estaba compuesta por 212 sujetos, de los cuales 160 eran mujeres y 52 varones. El rango de edad oscilaba entre 17 y 63 años, siendo la edad media de 34,40 años y la desviación típica de 9,17. Para análisis posteriores esta muestra fue dividida en 3 grupos de acuerdo con la edad: entre 17 y 24 años $(N=111)$, entre 25 y 40 años $(N=57)$ y entre 41 y 73 años $(N=34)$. En cuanto a los estudios, contamos con 16 personas con estudios elementales, 84 con estudios medios y 112 con estudios superiores. El $45,7 \%$ de la muestra eran psicólogos, o estaban en el último curso de sus estudios de psicologia; mientras que el $54,3 \%$ restante no eran psicólogos, ni cursaban estudios de Psicologia.

\section{Material y procedimiento}

Se construyó un listado de 125 palabras que se suelen utilizar en tareas experimentales sobre trastornos emocionales. Para seleccionar estas palabras se utilizaron dos fuentes diferentes. En primer lugar, se revisaron los listados de palabras incluídos en los artículos publicados en la literatura (tanto en lengua inglesa como española); hay que señalar, a este respecto, que en algunos de los articulos que utilizan material verbal, aunque se describe el tipo de estímulos empleados, no se incluye el listado específico de tales palabras o, en otros casos, se remiten al material utilizado por otros autores. En segundo lugar, se utilizaron los siguientes cuestionarios de ansiedad y depresión: el "Inventario de Depresión de Beck" (B.D.I.), la "Escala de Valoración Psiquiátrica de Hamilton para la Depresión", la "Escala de Auto-Calificación de Zung para la Depresión", el "Cuestionario de Ansiedad Estado-Rasgo" de Spielberger (S.T.A.I. E.-R), el "Inventario de Ansiedad de Beck" (B.A.l.), la "Escala de Valoración de la Ansiedad de Hamilton", la "Escala de Ansiedad Manifiesta" de Taylor (M.A.S.), el "Cuestiona- 
rio de Ansiedad Cognitivo-Somática" de Schwartz et al. (C.S.A.Q.) y el "Hospital Anxiety and Depression Scale" de Zigmond y Snaith (H.A.D.). A partir de estas fuentes se seleccionaron aquellas palabras que parecian cubrir, al menos en gran parte, el campo de las emociones de ansiedad y de depresión, descartando aquellas palabras que eran exclusivas de un único subtipo de estos trastornos (por ejemplo, se descartaron palabras como hospital $o$ ataque, que podrian ser amenazantes o emocionales exclusivamente para los sujetos con trastorno de angustia, o palabras sólo relacionadas con el contenido de obsesiones específicas, etc.).

Las palabras incluidas en el listado fueron ordenadas al azar para cada sujeto, de forma que no influyeran en el resultado factores como el cansancio de los sujetos. La tarea del sujeto consistia en señalar con una cruz de 0 a 10 (siendo $0=$ Nada y $10=$ Totalmente) el grado en que:

- el sujeto creía que esa palabra estaba relacionada con ansiedad;

- el sujeto creía que esa palabra estaba relacionada con depresión;

- el sujeto comprendia la palabra;

- la palabra podía evocarle una imagen; y, por último,

- con qué frecuencia el sujeto utilizaba cada una de las palabras.

\section{RESULTADOS}

En primer lugar hallamos la media y la desviación típica para cada una de las palabras en las cinco categorías de evaluación, a partir de las puntuaciones dadas por la muestra total. Estos resultados se encuentran en el Anexo 1.

En segundo lugar aplicamos diversas pruebas " $t$ " de Student para muestras independientes, con el fin de analizar las posibles diferencias entre varones y mujeres y entre ser o no psicólogo, en el uso, comprensión, imaginabilidad y relación con ansiedad y depresión de las palabras. Para analizar las posibles diferencias debidas a la edad, dividimos la muestra en tres grupos: sujetos entre los 17 y los 24 años, sujetos entre los 25 y los 40 años y sujetos entre 41 y 73 años. Posteriormente, se aplicaron diversos análisis de varianza unifactoriales para los tres grupos de edad para cada una de las palabras y las categorias. Para analizar las diferencias debidas al nivel de estudios, también dividimos la muestra en tres grupos: estudios elementales, estudios medios y estudios superiores. Las medias correspondientes a cada subdivisión de la muestra se encuentran en el Anexo 1. donde también se indica la presencia o no de diferencias significativas entre los grupos, tras aplicar los análisis estadisticos que acabamos de indicar.

Por último, también estábamos interesadas en saber si las palabras utilizadas diferian significativamente en su pertenencia a las dos categorias emocionales estudiadas (ansiedad y depresión). Para ello, aplicando la totalidad de la muestra, aplicamos diversas pruebas de " $t$ " de Student para muestras relacionadas. En el Anexo 2 se ofrece el listado tanto de las palabras para las que se han encontrado diferencias significativas, como las que no discriminan entre ambas emociones.

\section{DISCUSIÓN}

Las palabras, en general, se suelen utilizar como estimulos en un amplio número de tareas experimentales diseñadas para evaluar procesos psicológicos básicos (atención, percepción, etc.), estructuras cognitivas (autoesquemas, nodos de memoria, etc.), operaciones cognitivas (activación, facilitación, elaboración, etc.), etc. Este tipo de tareas se está utilizando cada vez más en el estudio sobre el funcionamiento cognitivo de los individuos con trastornos emocionales. Así, en estos últimos años estamos asistiendo a una gran proliferación de publicaciones sobre el rendimiento de estos sujetos en tareas como el Stroop modificado, tareas de memoria implicita y explícita, de detección de puntos, de autoadscripción de rasgos, de deletreo de homófonos, etc. En todas estas tareas es un requisito imprescindible el poder controlar determinadas variables de los estímulos verbales que se presentan. Nadie duda, por ejemplo, de la necesidad de equiparar la longitud de las palabras en una tarea de percepción subliminal, o de controlar la frecuencia de uso en una tarea de aprendizaje incidental. Qué variables concretas son las que se debe tener 
en cuenta dependerá de la naturaleza específica de la tarea experimental que se utilice. En general, se podria decir que la longitud, la frecuencia de uso, la imaginabilidad y la emocionalidad son las variables que la literatura suele señalar como relevantes en la mayoría de las tareas experimentales que se utilizan en el estudio de los trastornos emocionales. Los autores de lengua anglosajona cuentan con la ventaja de poseer un mayor número de diccionarios que oferten medidas de estas variables. Sin embargo, y como ya hemos señalado, en el idioma castellano no contamos apenas con estudios que analicen bases de palabras y aporten una baremación; y los que hay, como es el caso de Juilland y ChangRodríguez (1964) y Algarabel, Ruiz y Sanmartín (1988), no cubren totalmente el campo de las palabras "emocionales". El único trabajo que hemos encontrado en este sentido (Sanz, 1994) analiza palabras que son descriptivos de personalidad. Sin embargo, a veces el tipo de hipótesis que se plantea exige utilizar palabras que no sean adjetivos autodescriptivos, sino sustantivos, verbos, etc., cuyo contenido sea relevante para alguno de los trastornos emocionales. Por ejemplo, la palabra palpitación puede ser una palabra de contenido amenazante para un paciente con trastorno de pánico. Consideramos que es necesario contar con estudios en español sobre las características de estas palabras, ya que a veces su ausencia impide conocer y controlar variables tan importantes para el experimentador como la frecuencia de uso de los estímulos verbales. $Y$, precisamente, esa es la aportación del trabajo que aquí se presenta.

Obviamente, este trabajo es muy limitado ya que, por ejemplo, no oferta datos sobre palabras "neutras", necesarias en muchos estudios como estimulos controles, ni sobre palabras "específicas" de algunos trastornos (por ejemplo, suciedad, hospital, gente, etc.). Tampoco se ofertan datos sobre otras variables que podrian ser relevantes como "emocionalidad en general", "familiaridad", "diferentes significados posibles", etc. Por otro lado, somos plenamente conscientes de que la "frecuencia de uso" que se utiliza en este estudio no deja de ser una "frecuencia subjetiva de uso", ya que lo que se le pidió a los sujetos es que estimaran en qué grado consideraban que utilizaban esa palabra. Obviamente, a la hora de estimar la frecuencia de uso de un estimulo verbal existen otros métodos más rigurosos y precisos, como el vaciado de la literatura impresa en una lengua (utilizado por Juilland y Chang-Rodriguez), o el análisis del lenguaje televisivo, etc. Consideramos que tales análisis deberian hacerse y actualizarse periódicamente, sin embargo, tales metodologías excedian con creces los medios y propositos de nuestro trabajo, donde sólo pretendemos hacer una pequeña aportación a un campo restringido de estímulos verbales: las palabras emocionales.

Por otro lado, este estudio pone de manifiesto las diferencias existentes en el uso, comprensión, imaginabilidad y emocionalidad de las palabras, en función de las caracteristicas especificas de la muestra. Hemos encontrado que existen muchas diferencias en las cinco categorias en función de si el sujeto es psicólogo o no lo es. Parece que los psicólogos usan con más frecuencia estas palabras, las comprenden mejor, pueden evocar con mayor facilidad imágenes y las consideran más emocionales. Por tanto, aquellos estudios que utilizan sujetos procedentes de últimos cursos de Psicología deberían tener en cuenta estas observaciones. Además, esto también indicaría que cuando se trabaja con sujetos no psicólogos y no universitarios (como lo son la mayoria de los pacientes con trastornos emocionales) a la mejor no es muy conveniente utilizar exactamente el mismo material 0 , si se hace, se deben controlar las diferencias en estas variables. También hemos encontrado que las mujeres, en general, utilizan con más frecuencia algunas de las palabras emocionales estudiadas (en concreto 21 palabras), no habiendo substanciales diferencias para las otras categorias entre varones y mujeres. No tenemos una explicación concluyente para este resultado, aunque pensamos que quizás las diferencias educativas en cuanto a la expresión de emociones podrian ser responsables, al menos en parte, del mayor uso de palabras emocionales de las mujeres. Por lo que respecta a la edad de los sujetos, los individuos más mayores suelen utilizar con más frecuencia muchas de las palabras estudiadas, aunque son los jóvenes quienes más las relacionan con contenido emocional, especialmente 
con ansiedad. Por último, en cuanto al nivel de estudios, hemos encontrado muchas diferencias en todas las dimensiones, siendo el grupo con estudios superiores el más destacado en todas las categorias. Otro aspecto que consideramos especialmente interesante es saber hasta qué punto las palabras valoradas como emocionales pueden utilizarse indistintamente en estudios sobre depresión y sobre ansiedad. En este sentido, hemos encontrado que algunas de ellas parecen discriminar entre ambos tipos de emociones. Este aspecto es importante ya que algunos estudios (p.ej., Mathews y MacLeod, 1985; Tata et al., 1996) intentan discriminar si el rendimiento diferencial de los pacientes con trastornos emocionales en algunas tareas experimentales se produce porque los estímulos utilizados son emocionales, o si los estímulos verbales deben denotar un contenido emocional concreto para que se produzca esta ejecución diferencial. Aunque algunos autores, como por ejemplo McNally et al. (1994), han señalado que a la hora de estudiar un determinado trastomo emocional seria recomendable hacer una selección idiográfica de los estímulos, que asegure la relevancia emocional para cada sujeto, sin embargo, incluso en este caso. siempre seria conveniente el poder contar con un banco inicial de palabras que indique la emocionalidad general de esos estímulos e, incluso, que pueda discriminar entre emociones.

En conclusión, esperamos que este trabajo pueda ser de cierta utilidad para los investigadores interesados en el estudio experimental de los factores cognitivos involucrados en los trastornos emocionales, aportando información que a veces no es fácil de obtener, pero que supone un paso preliminar para poder utilizar el material estimular adecuado en diversos paradigmas experimentales.

\section{REFERENCIAS BIBLIOGRÁFICAS}

Algarabel, S., Ruiz, J.C. y Sanmartin, J. (1988). The University of Valencia's computerized word pool. Behavior Research Methods, Instruments \& Computers, 20, 398-403.

Belloch, A e lbáñez, E. (1991). Acerca del concepto de psicopatología. En A. Belloch y E.Ibáñez (Eds.)
Manual de Psicopatologia (vol.1) (pp. 1-46). Valencia: Promolibro.

Brewin, C.R. (1988). Cognitive foundations of Clinical Psychology. London: LEA.

Campos, A. y González, M.A. (1991). Imagery, meaningfulness and emotionality values of words when meaning is controlled. Perceptual and Motor Skills, 73, 787-791.

Carroll, J. B., Davies, P. y Richman, B. (1971). Word frequency book. New York: American Heritage

Eysenck, M.W. (1992). Anxiety: The cognitive perspective. London: Hillsdale.

Gotlib, I.H. y Cane, D.B. (1987). Construct accessibility and clinical depression: A longitudinal investigation. Joumal of Abnormal Psychology, 96, 199-204.

Graves, R., Landis, T. y Goodglass, H. (1981). Laterality and sex differences in visual recognition of emotional and non-emotional words. Neuropsychologia, 19, 95-102

Greenberg, M.S., Vázquez, C. y Alloy, L. B. (1988). Depression versus anxiety: Differences in self- and other-schemata. En L.B.Alloy (ed.) Cognitive processes in depression (pp. 109-142) New York: Guilford Press.

Juilland, A. y Chang-Rodríguez, E. (1964). Frequency dictionary of Spanish words. Mouton: The Hague.

Kucera, H. y Francis, W.N. (1967). Computational analysis of present-day American English. Providence, RI: Brown University Press.

Macleod, C. (1991). Half a century of research on the Stroop effect: An integrative review. Psychological Bulletin. 109, 163-203.

MacLeod, C. y Mathews, A. (1991). Biased cognitive operations in anxiety: accessibility of information or assignment of processing priorities. Behaviour Research and Therapy, 30, 599-610.

Martin, M., Williams, R.M. y Clark, D.M. (1991). Does anxiety lead to selective processing of threatrelated information?. Behaviour Research and Therapy, 29, 147-160.

Mathews, A. y Klug, F. (1993). Emotionality and interference with color-naming in anxiety. Behaviour Research and Therapy, 31, 57-62.

Mathews, A. y MacLeod. C. (1985). Selective processing of threat cues in anxiety disorders. Behaviour Research and Therapy, 23, 563-569.

Mogg, K., Bradley, B.P., Williams, R. y Mathews, A. (1993). Subliminal processing of emotional information in anxiety and depression. Joumal of Abnormal Psychology, 102, 304-311.

McNally, R.J., Amir, N., Louro, C.E., Lukach, B.M., Riemann, B.C. y Calamari, J.E. (1994). Cognitive processing of idiographic emotional information in panic disorder. Behaviour Research and Therapy, 32. 119-122 
Paivio, A. (1971). Imagery and verbal processes. New York: Holt, Rinehart \& Winston.

Pascual, J. (1984). Categorización de la información personal. Boletín de Psicologia, 4. 33-49.

Roget, P.M. (1962). Roget's International Thesaurus ( $3^{a}$ ed.). New York: Crowell.
Sanz, J. (1994). Autoesquemas depresivos y ansiosos. Se busca una estructura. Boletín de Psicología, 42, 75-107.

Tata P.R., Leibowitz, J.A., Prunty, M.J., Cameron, M. y Pickering, A.D. (1996). Attentional bias in obsessional compulsive disorder. Behaviour Research and Therapy, 34, 53-60

\section{NOTAS}

1 En el Anexo 1 no se incluyen los valores de las $F$ y de las $t$, ni los grados de libertad, para no hacer excesivamente larga la tabla. El lector interesado en estos datos puede dirigirse a las autoras del trabajo. 


\section{ANEXO 1: MEDIAS Y DESVIACIONES TIPICAS CORRESPONDIENTES A TODAS LAS PALABRAS}

ANEXO 1: MEDIAS Y DESVIACIONES TIPICAS CORRESPODIENTES A TODAS LAS PALABRAS

\begin{tabular}{|c|c|c|c|c|c|}
\hline & Frec. de uso & Imaginación & ensión & Ucpicsion & \\
\hline $\begin{array}{l}\text { Accidente }^{\text {(d) }} \\
\text { Mujer/Varón }^{\text {(b) }} \\
\text { Psic./NoPsi. }^{\text {(c) }} \\
{\text { Edad } 1 / 2 / \underline{3}^{\text {(d) }}}^{\text {Estu. } 1 / 2 / \underline{3}^{\text {(c) }}}\end{array}$ & $\begin{array}{l}7.35(2.88) \\
7.43 / 7.04 \\
8.08 / 6.74\left(^{*}\right) \\
7.64 / 6.35 / 8.13\left(^{*}\right) \\
7.77 / 7.29 / 7.41\end{array}$ & $\begin{array}{l}8.84(2.32) \\
8.92 / 8.58 \\
9.19 / 8.52\left(^{*}\right) \\
8.91 / 8.51 / 9.88 \\
8.31 / 8.97 / 8.60 \\
\end{array}$ & $\begin{array}{l}9.33(1.84) \\
9.43 / 9.00 \\
9.71 / 8.99(") \\
9.29 / 9.48 / 9.38 \\
9.62 / 9.15 / 9.64\end{array}$ & $\begin{array}{l}5.92(3.34) \\
6.10 / 5.34 \\
6.09 / 5.76 \\
6.23 / 5.00 / 4.88\left(^{*}\right) \\
5.00 / 6.25 / 5.41 \\
\end{array}$ & $\begin{array}{l}6.68(3.69) \\
6.85 / 6.10 \\
6.94 / 6.45 \\
6.94 / 6.27 / 4.50 \\
5.00 / 7.16 / 6.17\end{array}$ \\
\hline 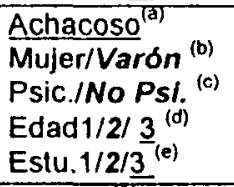 & $\begin{array}{l}2.67(2.92) \\
2.59 / 2.96 \\
2.78 / 2.61 \\
2.44 / 3.10 / 5.50\left(^{\star}\right) \\
3.54 / 2.35 / 3.19\left(^{\star}\right)\end{array}$ & \begin{tabular}{|l|}
$5.23(3.65)$ \\
$4.97 / 6.10\left(^{\star}\right)$ \\
$5.55 / 4.98$ \\
$4.66 / 6.72 / 7.88\left(^{\star}\right)$ \\
$5.17 / 4.65 / 6.45\left(^{\star}\right)$ \\
\end{tabular} & $\begin{array}{l}6.40(3.54) \\
6.33 / 6.63 \\
6.80 / 6.04 \\
5.83 / 7.73 / 9.00\left(^{*}\right) \\
5.69 / 5.92 / 7.53\left(^{\star}\right)\end{array}$ & $\begin{array}{l}4.72(3.50) \\
4.66 / 4.84 \\
5.55 / 4.03\left({ }^{\star}\right) \\
4.81 / 4.27 / 5.88 \\
4.38 / 4.65 / 4.98 \\
\end{array}$ & $\begin{array}{l}.09(3.14) \\
3.34 / 3.18 \\
3.27 / 3.35 \\
3.35 / 3.22 / 3.50 \\
3.69 / 3.33 / 3.25 \\
\end{array}$ \\
\hline 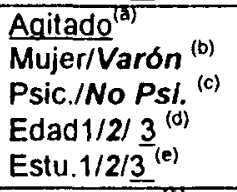 & \begin{tabular}{|l|}
$4.97(3.18)$ \\
$5.02 / 4.76$ \\
$5.20 / 4.76$ \\
$5.07 / 4.53 / 6.43$ \\
$5.31 / 4.81 / 5.29$ \\
\end{tabular} & $\begin{array}{l}7.13(2.82) \\
7.09 / 7.24 \\
7.36 / 6.93 \\
7.01 / 7.29 / 8.13 \\
7.54 / 6.97 / 7.34 \\
\end{array}$ & $\begin{array}{l}8.46(2.30) \\
8.68 / 7.78\left(^{\star}\right) \\
8.90 / 8.08\left(^{*}\right) \\
8.39 / 8.59 / 8.75 \\
7.38 / 8.26 / 9.08\left(^{*}\right) \\
\end{array}$ & $\begin{array}{l}3.00(3.08) \\
3.00 / 2.92 \\
2.77 / 3.14 \\
2.70 / 3.65 / 3.88 \\
3.23 / 2.84 / 3.38 \\
\end{array}$ & \begin{tabular}{|l|}
$7.80(2.86)$ \\
$7.85 / 7.63$ \\
$8.33 / 7.33\left({ }^{*}\right)$ \\
$7.93 / 7.20 / 8.63$ \\
$7.54 / 7.88 / 7.72$ \\
\end{tabular} \\
\hline 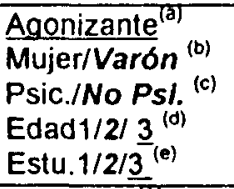 & $\begin{array}{l}3.64(3.15) \\
3.56 / 3.92 \\
4.02 / 3.33 \\
3.82 / 3.02 / 4.00 \\
3.23 / 3.47 / 4.05 \\
\end{array}$ & \begin{tabular}{|l|}
$7.12(2.98)$ \\
$7.09 / 7.22$ \\
$7.59 / 6.72\left(^{\star}\right)$ \\
$6.89 / 7.76 / 7.63$ \\
$5.38 / 6.95 / 7.79\left(^{\star}\right)$ \\
\end{tabular} & $\begin{array}{l}8.39(2.35) \\
8.42 / 8.31 \\
8.72 / 8.11 \\
8.30 / 8.80 / 8.63 \\
7.62 / 8.24 / 8.86 \\
\end{array}$ & $\begin{array}{l}5.12(3.63) \\
5.16 / 5.06 \\
5.03 / 5.24 \\
5.41 / 4.41 / 5.63 \\
5.77 / 5.26 / 4.81 \\
\end{array}$ & $\begin{array}{l}5.71(3.54) \\
5.75 / 5.65 \\
5.40 / 5.97 \\
6.23 / 4.47 / 5.13\left(^{*}\right) \\
5.23 / 5.84 / 5.64 \\
\end{array}$ \\
\hline 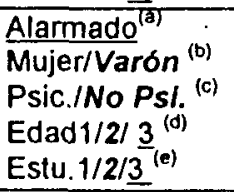 & $\begin{array}{l}4.91(3.13) \\
4.94 / 4.72 \\
5.60 / 4.27\left(^{(")}\right. \\
4.88 / 4.73 / 6.00 \\
5.31 / 4.76 / 5.00\end{array}$ & \begin{tabular}{l|}
$6.78(2.98)$ \\
$6.68 / 7.10$ \\
$6.93 / 6.66$ \\
$6.91 / 6.51 / 7.13$ \\
$6.54 / 6.82 / 6.67$ \\
\end{tabular} & $\begin{array}{l}(2.10) \\
/ 8.45 \\
/ 8.48 \\
/ 8.65 / 9.50 \\
/ 8.47 / 8.93\end{array}$ & $\begin{array}{l}3.25(3.00) \\
3.20 / 3.46 \\
3.07 / 3.41 \\
3.27 / 2.94 / 4.00 \\
3.54 / 3.27 / 3.14 \\
\end{array}$ & $\begin{array}{l}7.73(2.71) \\
7.78 / 7.53 \\
8.46 / 7.08\left(^{*}\right) \\
8.07 / 7.00 / 5.63\left(^{*}\right) \\
5.92 / 7.84 / 7.84\left(^{*}\right)\end{array}$ \\
\hline 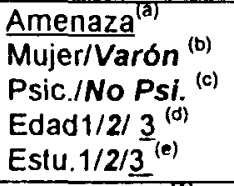 & $\begin{array}{l}5.02(3.31) \\
5.05 / 4.88 \\
5.95 / 4.20\left(^{\star}\right) \\
5.08 / 4.55 / 6.14 \\
5.08 / 4.68 / 5.71 \\
\end{array}$ & $\begin{array}{l}6.62(3.18) \\
6.60 / 6.65 \\
6.87 / 6.41 \\
6.76 / 6.61 / 4.88 \\
6.08 / 6.62 / 6.67 \\
\end{array}$ & $\begin{array}{l}(2.13) \\
/ 8.59 \\
/ 8.32\left(^{\star}\right) \\
/ 8.73 / 9.00 \\
/ 8.52 / 9.19 \\
\end{array}$ & $\begin{array}{l}(3.60) \\
4.39 \\
4.85 \\
4.21 / 3.63 \\
4.97 / 4.56 \\
\end{array}$ & $\begin{array}{l}7.82(2.86) \\
8.28 / 7.80 \\
8.67 / 7.07\left(^{*}\right) \\
8.15 / 7.31 / 4.38\left(^{*}\right) \\
6.00 / 8.28 / 8.38\left(^{*}\right)\end{array}$ \\
\hline 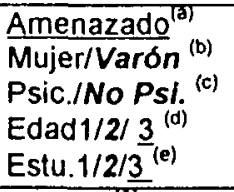 & $\begin{array}{l}5.18(3.16) \\
5.31 / 4.75 \\
6.00 / 4.49\left(^{\star}\right) \\
5.21 / 4.86 / 7.13 \\
4.69 / 4.82 / 6.10\left(^{*}\right) \\
\end{array}$ & \begin{tabular}{l|}
$7.33(2.78)$ \\
$7.39 / 7.22$ \\
$7.45 / 7.27$ \\
$7.07 / 7.88 / 9.13\left(^{*}\right)$ \\
$8.23 / 7.13 / 7.52$ \\
\end{tabular} & $\begin{array}{l}9.09(1.69) \\
9.11 / 9.04 \\
9.25 / 8.96 \\
8.99 / 9.43 / 9.50 \\
8.92 / 9.05 / 9.20 \\
\end{array}$ & $\begin{array}{l}(3.27) \\
/ 5.14 \\
/ 5.91 \\
/ 5.67 / 8.50\left(^{\star}\right) \\
/ 5.82 / 5.22\end{array}$ & $\begin{array}{l}8.14(2.75) \\
7.94 / 7.41 \\
8.68 / 7.07\left(^{*}\right) \\
8.36 / 7.86 / 7.00 \\
6.69 / 7.70 / 8.31 \\
\end{array}$ \\
\hline $\begin{array}{l}\text { Ansioso }^{(\mathrm{d})} \\
\text { Mujer/Varón } \\
{ }^{\text {(b) }} \\
\text { Psic./No Psl. } \\
\text { Edad } 1 / 2 / \underline{3}^{\text {(d) }} \\
{\text { Estu. } 1 / 2 / \underline{3}^{(\mathrm{e})}}\end{array}$ & $\begin{array}{l}6.07(3.27) \\
6.28 / 5.31 \\
7.01 / 5.23\left(^{*}\right) \\
6.31 / 5.38 / 5.43 \\
3.85 / 6.05 / 6.56\left(^{\star}\right)\end{array}$ & $\begin{array}{l}7.22(2.93) \\
7.23 / 7.18 \\
7.84 / 6.69 \\
7.40 / 6.88 / 7.00 \\
6.92 / 7.10 / 7.49 \\
\end{array}$ & $\begin{array}{l}8.49(2.23) \\
8.49 / 8.43 \\
8.69 / 8.27 \\
8.39 / 8.54 / 9.13\left(^{\star}\right) \\
7.00 / 8.44 / 8.83\left({ }^{*}\right) \\
\end{array}$ & $\begin{array}{l}3.90(3.63) \\
4.18 / 3.10 \\
3.25 / 4.49\left(^{*}\right) \\
3.46 / 5.21 / 4.88\left(^{*}\right) \\
4.31 / 3.95 / 3.84\end{array}$ & $\begin{array}{l}9.54(1.53) \\
9.67 / 9.12 \\
9.82 / 9.29\left(^{*}\right) \\
9.50 / 9.56 / 10.0\left(^{*}\right) \\
9.23 / 9.50 / 9.69 \\
\end{array}$ \\
\hline $\begin{array}{l}\text { Aprensión }^{(0)} \\
\text { Mujer/Varón }^{(b)} \\
\text { Psic./No Ps/. } \\
\text { Edad1/2/3 } \\
\text { Estu.1/2/3 }^{(0)}\end{array}$ & $\begin{array}{l}2.78(2.91) \\
2.84 / 2.60 \\
2.97 / 2.64 \\
2.35 / 3.57 / 5.00\left(^{\star}\right) \\
3.00 / 2.45 / 3.38 \\
\end{array}$ & \begin{tabular}{|l|}
$4.39(3.63)$ \\
$4.47 / 4.20$ \\
$4.43 / 4.41$ \\
$3.76 / 5.96 / 7.13\left(^{*}\right)$ \\
$6.54 / 3.74 / 5.32\left({ }^{*}\right)$ \\
\end{tabular} & $\begin{array}{l}6.70(3.34) \\
6.74 / 6.55 \\
7.00 / 6.41 \\
6.17 / 7.90 / 9.50\left(^{*}\right) \\
7.08 / 6.13 / \underline{7.78}\left({ }^{*}\right)\end{array}$ & \begin{tabular}{|l|}
$4.77(3.34)$ \\
$4.82 / 4.56$ \\
$5.14 / 4.42$ \\
$4.70 / 4.76 / 5.50$ \\
$5.54 / 4.82 / 4.59$ \\
\end{tabular} & $\begin{array}{l}6.22(3.46) \\
6.17 / 6.32 \\
6.62 / 5.85 \\
6.16 / 6.31 / 5.88 \\
5.85 / 6.14 / 6.57 \\
\end{array}$ \\
\hline 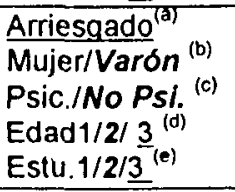 & \begin{tabular}{|l|}
$6.04(2.93)$ \\
$6.02 / 6.06$ \\
$6.67 / 5.50\left(^{\star}\right)$ \\
$6.23 / 5.24 / 7.25$ \\
$6.08 / 5.85 / 6.39$ \\
\end{tabular} & $\begin{array}{l}7.67(2.72) \\
7.43 / 8.33\left(^{\star}\right) \\
7.54 / 7.75 \\
7.76 / 7.31 / 7.50 \\
8.55 / 7.98 / 7.25\left(^{*}\right)\end{array}$ & $\begin{array}{l}8.70(2.14) \\
8.68 / 8.78 \\
8.88 / 8.54 \\
8.75 / 8.53 / 8.50 \\
7.38 / 8.78 / 8.75 \\
\end{array}$ & $\begin{array}{l}1.90(2.52) \\
2.01 / 1.55 \\
2.09 / 1.71 \\
1.90 / 1.94 / 1.63 \\
2.69 / 1.60 / 2.41\end{array}$ & $\begin{array}{l}6.59(3.39) \\
6.57 / 6.61 \\
7.39 / 5.88\left(^{\star}\right) \\
6.93 / 5.67 / 5.25\left(^{*}\right) \\
6.38 / 6.76 / 6.24 \\
\end{array}$ \\
\hline
\end{tabular}




\begin{tabular}{|c|c|c|c|c|c|}
\hline & Frec. de uso & Imaginación & Comprensión & Depresión & Ansiedad \\
\hline 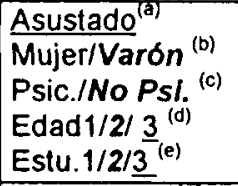 & \begin{tabular}{|l|}
$6.52(3.14)$ \\
$6.84 / 5.46\left(^{*}\right)$ \\
$7.21 / 5.91\left(^{*}\right)$ \\
$6.75 / 5.73 / 6.86$ \\
$5.92 / 6.40 / 6.90$ \\
\end{tabular} & \begin{tabular}{|l|}
$7.87(2.53)$ \\
$7.86 / 7.86$ \\
$8.07 / 7.67$ \\
$7.88 / 7.94 / 8.38$ \\
$7.31 / 7.80 / 8.07$ \\
\end{tabular} & \begin{tabular}{|l|}
$8.96(1.89)$ \\
$9.03 / 8.76$ \\
$9.21 / 8.75$ \\
$8.94 / 9.10 / 9.38$ \\
$8.62 / 8.82 / 9.33$ \\
\end{tabular} & \begin{tabular}{|l|}
$5.21(3.64)$ \\
$5.26 / 5.15$ \\
$5.35 / 5.16$ \\
$5.45 / 4.67 / 5.25$ \\
$4.46 / 5.42 / 5.07$ \\
\end{tabular} & \begin{tabular}{|l|}
$8.08(2.57)$ \\
$8.13 / 7.88$ \\
$8.40 / 7.81$ \\
$8.28 / 7.90 / 5.13\left({ }^{*}\right)$ \\
$6.54 / 8.09 / 8.45$ \\
\end{tabular} \\
\hline 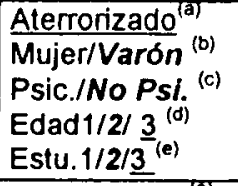 & \begin{tabular}{|l|}
$5.35(3.25)$ \\
$5.56 / 4.63$ \\
$6.10 / 4.69\left(^{*}\right)$ \\
$5.65 / 4.53 / 4.88$ \\
$4.62 / 5.18 / 5.85$ \\
\end{tabular} & \begin{tabular}{|l|}
$8.31(2.31)$ \\
$8.28 / 8.39$ \\
$8.68 / 7.97\left(^{*}\right)$ \\
$8.39 / 8.10 / 8.00$ \\
$7.31 / 8.31 / 8.45$ \\
\end{tabular} & \begin{tabular}{|l|}
$8.91(2.02)$ \\
$8.84 / 9.12$ \\
$9.21 / 8.64\left({ }^{*}\right)$ \\
$9.02 / 8.63 / 8.63$ \\
$7.62 / 8.95 / 9.07$ \\
\end{tabular} & \begin{tabular}{|l|}
$4.81(3.57)$ \\
$4.91 / 4.55$ \\
$4.50 / 5.11$ \\
$4.90 / 4.69 / 5.25$ \\
$6.23 / 4.91 / 4.38$ \\
\end{tabular} & \begin{tabular}{|l|}
$8.54(2.47)$ \\
$8.64 / 8.22$ \\
$8.86 / 8.24$ \\
$8.68 / 8.29 / 7.38$ \\
$6.92 / 8.60 / 8.76(*)$ \\
\end{tabular} \\
\hline 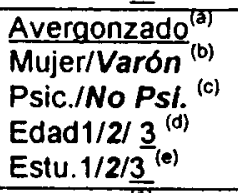 & \begin{tabular}{|l|}
$6.86(2.83)$ \\
$7.13 / 6.00\left({ }^{*}\right)$ \\
$7.34 / 6.47\left({ }^{*}\right)$ \\
$7.15 / 5.98 / 7.75\left(^{*}\right)$ \\
$7.69 / 6.80 / 6.81$ \\
\end{tabular} & \begin{tabular}{|l|}
$7.53(3.10)$ \\
$7.57 / 7.41$ \\
$7.90 / 7.26$ \\
$7.56 / 7.49 / 8.50$ \\
$7.08 / 7.38 / 7.98$ \\
\end{tabular} & \begin{tabular}{|l|}
$9.11(1.95)$ \\
$9.13 / 9.06$ \\
$9.51 / 8.76\left({ }^{*}\right)$ \\
$9.19 / 9.06 / 8.75$ \\
$9.08 / 9.04 / 9.25$ \\
\end{tabular} & \begin{tabular}{|l|}
$6.19(3.10)$ \\
$6.12 / 6.44$ \\
$6.40 / 6.04$ \\
$6.19 / 6.24 / 6.63$ \\
$5.77 / 6.17 / 6.55$ \\
\end{tabular} & $\left.\begin{array}{|l|}6.27(3.24) \\
6.41 / 5.82 \\
6.28 / 6.22 \\
6.46 / 5.86 / 5.38 \\
3.92 / 6.49 / 6.41 \\
\end{array}{ }^{*}\right)$ \\
\hline 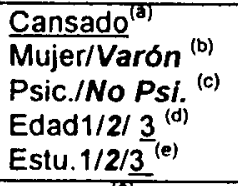 & $\begin{array}{l}3) \\
(") \\
9.13 \\
8.41 \\
\end{array}$ & $\begin{array}{l}8.14(2.92) \\
8.13 / 8.18 \\
8.51 / 7.86 \\
8.23 / 7.96 / 9.13 \\
7.85 / 8.01 / 8.52 \\
\end{array}$ & $\begin{array}{l}9.09(2.20) \\
9.07 / 9.16 \\
9.48 / 8.76 \\
9.17 / 8.96 / 9.63 \\
9.15 / 9.01 / 9.25 \\
\end{array}$ & \begin{tabular}{|l|}
$6.52(3.67)$ \\
$6.73 / 5.84$ \\
$7.50 / 5.67\left(^{*}\right)$ \\
$6.65 / 6.45 / 6.63$ \\
$6.69 / 6.34 / 7.00$ \\
\end{tabular} & \begin{tabular}{|l|}
$3.49(3.37)$ \\
$3.65 / 3.06$ \\
$3.19 / 3.78$ \\
$3.54 / 3.67 / 2.38$ \\
$4.54 / 3.64 / 3.02$ \\
\end{tabular} \\
\hline 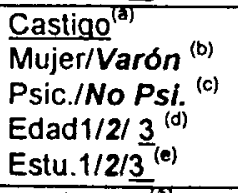 & \begin{tabular}{|l|}
$5.65(3.42)$ \\
$5.68 / 5.51$ \\
$6.52 / 4.88\left({ }^{\circ}\right)$ \\
$5.89 / 4.82 / 6.50$ \\
$5.08 / 5.55 / 5.97$ \\
\end{tabular} & $\begin{array}{l}7.77(2.81) \\
7.69 / 7.55 \\
7.92 / 7.43 \\
7.64 / 7.65 / 7.25 \\
5.92 / 7.79 / 7.72 \\
\end{array}$ & \begin{tabular}{|l}
$8.82(2.09)$ \\
$8.83 / 8.78$ \\
$9.09 / 8.57$ \\
$8.85 / 8.88 / 8.63$ \\
$7.69 / 8.76 / 9.17$ \\
\end{tabular} & \begin{tabular}{|l|}
$5.26(3.64)$ \\
$5.24 / 5.45$ \\
$5.44 / 5.14$ \\
$\left.5.69 / 4.37 / 4.00{ }^{\circ}\right)$ \\
$3.77 / 5.58 / 4.96$ \\
\end{tabular} & \begin{tabular}{|l|}
$5.15(3.51)$ \\
$5.27 / 4.84$ \\
$5.36 / 5.03$ \\
$5.62 / 3.84 / 4.38\left({ }^{*}\right)$ \\
$3.62 / 5.89 / 3.90$ \\
\end{tabular} \\
\hline 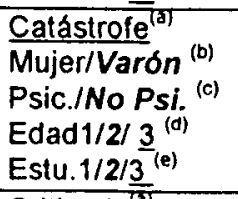 & \begin{tabular}{|l|}
$5.63(3.14)$ \\
$5.68 / 5.43$ \\
$6.18 / 5.16\left(^{*}\right)$ \\
$5.81 / 4.77 / 7.38\left({ }^{*}\right)$ \\
$6.08 / 5.38 / 6.08$ \\
\end{tabular} & $\begin{array}{l}8.60(2.33) \\
8.67 / 8.37 \\
8.92 / 8.32 \\
8.70 / 8.18 / 10.00 \\
7.46 / 8.74 / 8.48 \\
\end{array}$ & $\begin{array}{l}9.00(1.98) \\
9.03 / 8.86 \\
9.35 / 8.68\left({ }^{*}\right) \\
8.93 / 9.20 / 9.75 \\
9.38 / 8.88 / 9.14 \\
\end{array}$ & $\begin{array}{l}6.37(3.48) \\
6.82 / 5.02\left(^{*}\right) \\
6.57 / 6.21 \\
6.66 / 5.51 / 6.63 \\
6.77 / 6.39 / 6.31 \\
\end{array}$ & $\begin{array}{l}6.85(3.50) \\
7.03 / 6.27 \\
7.28 / 6.44 \\
7.22 / 5.86 / 6.00\left({ }^{*}\right) \\
5.15 / 7.08 / 6.84 \\
\end{array}$ \\
\hline 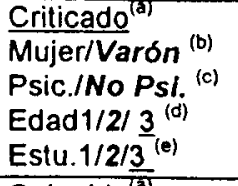 & \begin{tabular}{|l}
$6.17(3.23)$ \\
$6.25 / 5.82$ \\
$6.90 / 5.52\left({ }^{*}\right)$ \\
$6.32 / 5.47 / 7.13$ \\
$5.77 / 6.01 / 6.55$ \\
\end{tabular} & $\begin{array}{l}7.03(3.08) \\
7.04 / 7.10 \\
6.90 / 7.20 \\
6.99 / 7.58 / 5.38 \\
6.77 / 6.99 / 7.12 \\
\end{array}$ & $\begin{array}{l}8.87(1.95) \\
8.86 / 8.88 \\
9.15 / 8.61\left(^{*}\right) \\
8.87 / 8.98 / 8.75 \\
7.62 / 8.84 / 9.14\left(^{*}\right) \\
\end{array}$ & \begin{tabular}{|l|}
$5.78(3.55)$ \\
$5.57 / 6.57$ \\
$6.13 / 5.52$ \\
$6 / 12 / 4.85 / 5.38$ \\
$3.15 / 6.02 / 5.84\left(^{*}\right)$ \\
\end{tabular} & \begin{tabular}{|l|}
$5.80(3.60)$ \\
$5.96 / 5.42$ \\
$5.95 / 5.75$ \\
$6.14 / 4.65 / 6.25\left({ }^{*}\right)$ \\
$4.85 / 6.22 / 5.12$ \\
\end{tabular} \\
\hline $\begin{array}{l}\text { Culpable }^{(j)} \\
\text { Mujer/Varón }^{(\mathrm{d})} \\
\text { Psic./No Psi. }^{(\mathrm{c})} \\
\text { Edad11/2/ }^{\text {(d) }} \\
\text { Estu. } 1 / 2 / \underline{3}^{(\mathrm{e})}\end{array}$ & \begin{tabular}{|l|}
$5.99(3.16)$ \\
$6.09 / 5.63$ \\
$6.80 / 5.30\left({ }^{*}\right)$ \\
$6.38 / 4.80 / 6.50\left({ }^{*}\right)$ \\
$5.46 / 5.90 / 6.37$ \\
\end{tabular} & $\begin{array}{l}6.86(3.05) \\
6.68 / 7.38 \\
6.81 / 6.90 \\
6.76 / 6.78 / 8.88 \\
7.00 / 6.91 / 6.66 \\
\end{array}$ & $\begin{array}{l}8.96(1.92) \\
8.98 / 8.92 \\
9.24 / 8.72\left({ }^{*}\right) \\
9.00 / 8.86 / 8.63 \\
7.62 / 8.96 / 9.22\left({ }^{*}\right) \\
\end{array}$ & $\begin{array}{l}3.28) \\
.49 \\
.64 \\
.41 / 5.75 \\
.98 / 7.26 \\
\end{array}$ & \begin{tabular}{|l|}
$6.52(3.48)$ \\
$6.47 / 6.67$ \\
$6.17 / 6.85$ \\
$6.43 / 6.51 / 8.25$ \\
$7.54 / 6.93 / 5.38(*)$ \\
\end{tabular} \\
\hline 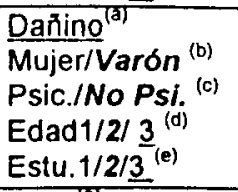 & \begin{tabular}{|l|}
$4.95(3.21)$ \\
$5.14 / 4.31$ \\
$5.63 / 4.40\left({ }^{*}\right)$ \\
$4.90 / 4.90 / 6.38$ \\
$4.23 / 4.76 / 5.54$ \\
\end{tabular} & $\begin{array}{l}6.63(3.38) \\
6.56 / 6.88 \\
6.65 / 6.66 \\
6.50 / 7.02 / 7.75 \\
6.46 / 6.58 / 6.76 \\
\end{array}$ & $\begin{array}{l}8.67(2.42) \\
8.69 / 8.61 \\
9.13 / 8.27\left(^{*}\right) \\
8.72 / 8.47 / 9.38 \\
7.15 / 8.66 / 8.97\left({ }^{*}\right) \\
\end{array}$ & \begin{tabular}{|l}
$4.30(3.32)$ \\
$4.53 / 3.59$ \\
$4.32 / 4.28$ \\
$4.57 / 3.82 / 2.88$ \\
$3.15 / 4.42 / 4.31$ \\
\end{tabular} & \begin{tabular}{|l|}
$5.41(3.57)$ \\
$5.48 / 5.10$ \\
$6.05 / 4.83\left(^{*}\right)$ \\
$5.76 / 4.55 / 3.00\left(^{*}\right)$ \\
$4.31 / 5.55 / 5.40$ \\
\end{tabular} \\
\hline 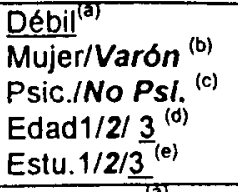 & \begin{tabular}{|l|}
$6.57(3.08)$ \\
$6.68 / 6.16$ \\
$7.01 / 6.19$ \\
$6.85 / 5.80 / 6.75$ \\
$7.00 / 6.39 / 6.88$ \\
\end{tabular} & $\begin{array}{l}7.29(2.96) \\
7.44 / 6.86 \\
7.66 / 7.03 \\
7.36 / 7.57 / 6.50 \\
6.62 / 7.26 / 7.50 \\
\end{array}$ & $\begin{array}{l}8.90(1.91) \\
8.91 / 8.86 \\
9.26 / 8.58\left({ }^{*}\right) \\
8.97 / 8.88 / 8.63 \\
8.00 / 8.85 / 9.15 \\
\end{array}$ & \begin{tabular}{|l|}
$6.57(3.26)$ \\
$6.51 / 6.76$ \\
$7.46 / 5.83\left({ }^{*}\right)$ \\
$6.81 / 6.06 / 4.75$ \\
$5.77 / 6.57 / 6.79$ \\
\end{tabular} & \begin{tabular}{|l|}
$3.35(3.06)$ \\
$3.37 / 3.33$ \\
$3.21 / 3.50$ \\
$3.22 / 3.86 / 3.75$ \\
$5.46 / 3.27 / 3.19$ \\
\end{tabular} \\
\hline 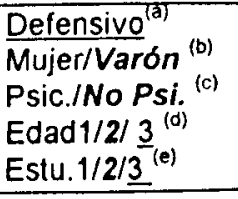 & \begin{tabular}{|l|}
$4.42(3.09)$ \\
$4.34 / 4.65$ \\
$5.09 / 3.87\left(^{*}\right)$ \\
$4.37 / 4.31 / 6.00$ \\
$4.23 / 4.29 / 4.64$ \\
\end{tabular} & $\begin{array}{l}5.72(3.28) \\
5.65 / 6.02 \\
5.83 / 5.69 \\
5.75 / 5.88 / 5.38 \\
5.46 / 5.72 / 5.72 \\
\end{array}$ & $\begin{array}{l}8.07(2.46) \\
8.05 / 8.12 \\
8.14 / 7.99 \\
8.03 / 8.20 / 8.78 \\
7.15 / 7.92 / 8.56 \\
\end{array}$ & $\begin{array}{l}4.22(3.42) \\
4.04 / 4.69 \\
4.46 / 4.00 \\
4.21 / 4.22 / 4.00 \\
3.31 / 4.30 / 4.33 \\
\end{array}$ & \begin{tabular}{|l|}
$6.04(3.40)$ \\
$6.13 / 5.80$ \\
$6.60 / 5.56\left({ }^{*}\right)$ \\
$6.08 / 6.08 / 4.38$ \\
$6.15 / 6.00 / 6.12$ \\
\end{tabular} \\
\hline
\end{tabular}




\begin{tabular}{|c|c|c|c|c|c|}
\hline & Frec. de uso & Imaginación & Comprensión & Depresión & Ansiedad \\
\hline Deficiente $^{\left(x^{\prime}\right)}$ & $4.53(3.25)$ & $6.48(3.41)$ & $8.30(2.51)$ & $3.85(3.74)$ & $2.76(3.17)$ \\
\hline Mujer/Varón ${ }^{\text {(b) }}$ & $4.64 / 4.12$ & $6.41 / 6.67$ & $8.47 / 7.73$ & $3.62 / 4.67$ & $2.79 / 2.71$ \\
\hline Psic./No Psl. ${ }^{(c)}$ & $5.58 / 3.60$ & $6.68 / 6.28$ & $8.48 / 8.11$ & $3.52 / 4.19$ & $2.66 / 2.88$ \\
\hline Edad1/2/ $\underline{3}^{(0)}$ & $4.61 / 4.14 / 4.86$ & $6.43 / 6.73 / \underline{5.75}$ & $8.19 / 8.53 / \underline{8.50}$ & $3.93 / 3.39 / \underline{5.63}$ & $2.87 / 2.22 / 3.50$ \\
\hline Estu.1/2/3 ${ }^{(e)}$ & $4.31 / 4.22 / 5.17$ & $6.08 / 6.48 / 6.44$ & $7.62 / 8.19 / 8.60$ & $\left.3.46 / 4.30 / 2.84()^{*}\right)$ & $3.31 / 3.09 / 1.74\left(0^{\circ}\right)$ \\
\hline Deprimido $^{(0)}$ & $7.06(2.89)$ & $7.57(2.86)$ & $8.94(1.79)$ & $9.34(1.98)$ & $4.72(3.75)$ \\
\hline Mujer/Varón ${ }^{(\mathrm{b})}$ & $7.30 / 6.28\left(^{\star}\right)$ & $7.56 / 7.65$ & $8.98 / 8.82$ & $9.23 / 9.69$ & $4.82 / 4.47$ \\
\hline Psic./No Psl. ${ }^{(c)}$ & $7.64 / 6.58\left(^{*}\right)$ & $7.74 / 7.42$ & $8.96 / 8.92$ & $9.63 / 9.09\left(^{*}\right)$ & $3.76 / 5.57\left(^{\circ}\right)$ \\
\hline Edad1/2/ $\underline{3}_{(\mathrm{e})}^{(\mathrm{d})}$ & $7.29 / 6.43 / 7.14$ & $7.54 / 7.69 / 7.50$ & $8.88 / 9.08 / \underline{9.13}$ & $9.46 / 9.10 / 8.50$ & $4.37 / 5.94 / 4.63\left(^{*}\right)$ \\
\hline Estu.1/2/3 ${ }^{(e)}$ & $6.31 / 6.92 / 7.47$ & $7.00 / 7.34 / 8.10$ & $8.38 / 8.81 / \overline{9.32}$ & $8.46 / 9.32 / 9.55$ & $5.54 / 4.94 / 4.17\left({ }^{*}\right)$ \\
\hline Desamparado & $4.35(3.30)$ & $6.71(3.18)$ & $8.67(2.15)$ & $7.54(3.20)$ & $5.45(3.66)$ \\
\hline Mujer/Varón ${ }^{(\mathrm{b})}$ & $4.49 / 3.82$ & $6.80 / 6.48$ & $8.78 / 8.33$ & $7.44 / 7.84$ & $5.65 / 4.92$ \\
\hline Psic./No Psi. ${ }^{\text {(c) }}$ & $4.59 / 4.11$ & $6.97 / 6.51$ & $8.98 / 8.40\left({ }^{*}\right)$ & $7.89 / 7.23$ & $4.83 / 6.03\left({ }^{\circ}\right)$ \\
\hline $1 / 2 / \underline{3}^{(\mathrm{d})}$ & $4.37 / 3.73 / 7.75\left(^{*}\right)$ & $6.73 / 6.78 / 7.00$ & $8.64 / 8.92 / 8.88$ & $7.91 / 6.55 / \underline{6.38}\left(^{*}\right)$ & $5.42 / 5.53 / 5.88$ \\
\hline $13^{(e)}$ & $6.31 / 4.12 / 4.53$ & $6.08 / 6.68 / \overline{6.84}$ & $4 \longdiv { 9 . 1 2 }$ & $6.38 / 7.73 / 7.29$ & $6.08 / 5.72 / \overline{4.74}$ \\
\hline Desanimado $^{(0)}$ & $6.64(2.77)$ & $7.36(.89)$ & $8.84(2.04)$ & $8.82(2.28)$ & $4.26(3.50)$ \\
\hline Varón ${ }^{(0)}$ & $6.81 / 6.49$ & $7.30 / 7.59$ & .67 & .65 & $4.33 / 4.12$ \\
\hline Psic./No Psi. ${ }^{\text {(c) }}$ & $7.23 / 6.33\left(^{\star}\right)$ & $7.46 / 7.34$ & $8.97 / 8.72$ & $9.20 / 8.48\left({ }^{\star}\right)$ & $3.98 / 4.52$ \\
\hline Edad1/2/ $\underline{3}^{(\mathrm{d})}$ & $6.89 / 6.14 / 7.63$ & $7.39 / 7.55 / 6.63$ & $8.73 / 9.20 / 8.38$ & $8.87 / 8.73 / 7.75$ & $3.97 / 5.14 / 5.00$ \\
\hline Estu & $7.62 / 6.46 / 7.12$ & $6.00 / 7.39 / 7.60$ & $5 \longdiv { 8 . 8 6 }$ & $8.92 / 8.69 / 9.14$ & $4.92 / 4.33 / 4.09$ \\
\hline Desastre $^{\left(\theta^{(1)}\right.}$ & $6.77(2.97)$ & $7.67(296)$ & $8.64(2.29)$ & $5.78(3.75)$ & $5.15(3.80)$ \\
\hline$\overline{V a r o ́ n}^{(b)}$ & $7.03 / 5.94\left(^{\star}\right)$ & $7.75 / 7.47$ & 8.6 & .78 & 5.28 \\
\hline Psic./No Psi. ${ }^{(c)}$ & $7.22 / 6.39\left(^{*}\right)$ & $7.98 / 7.43$ & $9.01 / 8.34\left(^{*}\right)$ & $6.37 / 5.31\left(^{*}\right)$ & $5.49 / 4.83$ \\
\hline Edad1/2/ $\underline{3}^{(\mathrm{d})}$ & $7.02 / 5.94 / 8.13\left(^{*}\right)$ & $7.80 / 7.33 / \underline{7.63}$ & $8.56 / 8.84 / 9.25$ & $5.96 / 5.49 / \underline{4.63}$ & $5.22 / 4.51 / 7.25$ \\
\hline Estu & $6.85 / 6.73 / 6.88$ & $6.46 / 7.59 / 8.07$ & $8.23 / 8.54 / 8.95$ & $5.08 / 5.73 / 5.98$ & $3.92 / 5.40 / 4.91$ \\
\hline Desconfiado & $6.76(2.93)$ & $6.36(3.44)$ & $8.97(2.12)$ & $4.18(3.35)$ & $6.00(3.36)$ \\
\hline $\operatorname{Var\delta ́n}^{(b)}$ & $6.99 / 6.00\left(^{\star}\right)$ & $6.69 / 7.00$ & $8.92 / 9.10$ & $4.10 / 4.47$ & $6.03 / 5.96$ \\
\hline Psic./No Psi. ${ }^{(c)}$ & $7.33 / 6.29\left(^{*}\right)$ & $6.75 / 6.82$ & $9.44 / 8.55\left({ }^{\star}\right)$ & $4.38 / 4.00$ & $6.80 / 5.35\left(^{*}\right)$ \\
\hline Edad $1 / 2 / \underline{3}^{(0)}$ & $7.04 / 5.92 / \underline{6.50}$ & $6.72 / 7.17 / \underline{6.00}$ & $8.95 / 8.94 / 9.50$ & $4.05 / 4.73 / 4.00$ & $6.31 / 5.37 / 3.88\left(^{*}\right)$ \\
\hline $2 / \underline{3}^{(e)}$ & $6.69 / 6.65 / \overline{7.03}$ & $7.77 / 6.59 / 6.91$ & $9.46 / 8.79 / 9.22$ & $5.69 / 4.20 / 3.84$ & $\left.4.15 / 6.37 / 5.600^{\circ}\right)$ \\
\hline Descuidado $^{(\mathrm{a})}$ & $6.46(2.72)$ & $7.33(3.03)$ & $8.59(2.30)$ & $4.30(3.76)$ & $2.75(3.01)$ \\
\hline arón ${ }^{(b)}$ & $6.46 / 6.51$ & $7.26 / 7.67$ & 8.5 & $4.37 / 4.06$ & $2.80 / 2.65$ \\
\hline Psic./No PsI. ${ }^{(c)}$ & $6.81 / 6.20$ & $7.66 / 7.15$ & $9.03 / 8.22\left({ }^{*}\right)$ & $5.47 / 3.30\left(^{\circ}\right)$ & $2.88 / 2.66$ \\
\hline $2 / \underline{3}^{(\mathrm{d})}$ & $6.77 / 5.55 / \underline{68}\left(^{*}\right)$ & $7.48 / 7.21 / \underline{.75}$ & $8.65 / 8.47 / 9.00$ & $4.54 / 3.82 / 4.13$ & $2.92 / 2.53 / 1.25$ \\
\hline $1 \underline{3}^{(e)}$ & $5.92 / 6.33 / 6.85$ & $5.15 / 7.38 / 7.74\left(^{*}\right)$ & $7.69 / 8.50 / 8.92$ & $2.92 / 3.88 / \overline{5.78}\left({ }^{*}\right)$ & $1.92 / 2.91 / 2.64$ \\
\hline Desdeñado & $1.74(2.35)$ & $4.17(3.46)$ & $5.77(3.54)$ & $4.74(3.73)$ & $3.29(3.32)$ \\
\hline Mujer/Varón ${ }^{(b)}$ & $1.67 / 1.92$ & $4.04 / 4.68$ & 5.85 & $4.75 / 4.62$ & $3.24 / 3.51$ \\
\hline Psic./No Psi. ${ }^{\text {(c) }}$ & $1.96 / 1.54$ & $4.51 / 3.89$ & $6.34 / 5.23\left(^{\star}\right)$ & $5.79 / 3.77\left(^{*}\right)$ & $3.49 / 3.16$ \\
\hline $12 / \underline{3}^{(d)}$ & $1.60 / 1.98 / 2.75$ & $3.97 / 4.65 / 5.50$ & $5.42 / 6.49 / 7.50$ & $4.66 / 4.49 / 6.75$ & $3.29 / 3.20 / 4.00$ \\
\hline Estu.1/2/3 ${ }^{(e)}$ & $1.85 / 1.63 / 2.00$ & $4.08 / 3.83 / 4.81$ & $4.92 / 5.23 / 7.05\left(^{*}\right)$ & $4.46 / 4.37 / 5.60$ & $3.31 / 3.37 / 3.00$ \\
\hline Desdichado $^{(d)}$ & $4.18(3.29)$ & $6.75(3.18)$ & $8.95(1.84)$ & $8.65(2.38)$ & $5.46(3.63)$ \\
\hline Mujer/Varón ${ }^{\text {(b) }}$ & $4.26 / 3.88$ & $6.69 / 6.92$ & $9.03 / 8.69$ & $8.68 / 8.53$ & $5.42 / 5.71$ \\
\hline Psic./No Psi. ${ }^{\text {(c) }}$ & $4.42 / 3.98$ & $6.89 / 6.67$ & $9.29 / 8.65\left(^{*}\right)$ & $9.07 / 8.27\left(^{*}\right)$ & $4.73 / 6.12\left(^{\circ}\right)$ \\
\hline $1 / 2 / \underline{3}^{(d)}$ & $4.16 / 4.00 / 6.00$ & $6.67 / 6.76 / 8.63$ & $8.83 / 9.31 / \underline{9.63}$ & $8.74 / 8.43 / 8.00$ & $5.38 / 6.00 / 4.63$ \\
\hline Estu.1/2/3 ${ }^{(e)}$ & $5.15 / 3.85 / \overline{4.76}$ & $7.69 / 6.48 / 7.17$ & $9.15 / 8.76 / 9.32$ & $7.62 / 8.62 / 8.98$ & $6.46 / 5.72 / \overline{4.74}$ \\
\hline Desesperado & $5.76(3.30)$ & $7.36(3.01)$ & $8.86(2.02)$ & $7.53(3.29)$ & $8.63(2.19)$ \\
\hline Mujer/Varón ${ }^{\text {(b) }}$ & $6.04 / 4.84\left(^{\star}\right)$ & $7.36 / 7.43$ & $8.92 / 8.69$ & $7.66 / 7.22$ & $8.63 / 8.63$ \\
\hline Psic./No Psi. ${ }^{(\mathrm{c})}$ & $6.52 / 5.14\left(^{\circ}\right)$ & $7.39 / 7.42$ & $9.05 / 8.70$ & $7.66 / 7.44$ & $8.52 / 8.73$ \\
\hline $\operatorname{Edad} 1 / 2 / 3^{(\mathrm{d})}$ & $6.02 / 4.88 / 7.13$ & $7.18 / 7.92 / 8.88$ & $8.70 / 9.41 / 8.88$ & $7.46 / 7.86 / 7.75$ & $8.66 / 8.63 / 7.88$ \\
\hline Estu.1/2/3 ${ }^{(e)}$ & $5.38 / 5.57 / 6.34$ & $7.69 / 7.16 / 7.76$ & $8.54 / 8.69 / 9.29$ & $6.99 / 7.28 / 8.25$ & $9.31 / 8.77 / 8.22$ \\
\hline Desgracia $^{(\text {t) }}$ & $6.29(3.16)$ & $7.52(2.90)$ & $8.87(1.99)$ & $8.45(2.51)$ & $5.18(3.61)$ \\
\hline Mujer/Varón ${ }^{\text {(b) }}$ & $6.35 / 6.06$ & $7.30 / 8.18$ & $8.87 / 8.88$ & $8.41 / 8.53$ & $5.08 / 5.59$ \\
\hline Psic./No Psi. (c) & $6.75 / 5.87\left(^{*}\right)$ & $7.48 / 7.52$ & $9.19 / 8.59\left(^{*}\right)$ & $8.79 / 8.13$ & $4.72 / 5.59$ \\
\hline Edad1/2/ $\underline{3}^{(\mathrm{d})}$ & $6.47 / 5.40 / 8.00\left(^{*}\right)$ & $7.48 / 7.73 / \underline{6} 8 \underline{8}$ & $8.92 / 8.63 / 9.13$ & $8.66 / 7.88 / 7.63$ & $5.19 / 5.45 / 4.63$ \\
\hline Estu. 1/2/3 ${ }^{(e)}$ & $7.18 / 6.02 / 6.63$ & $6.09 / 7.49 / 7.76$ & $7.64 / 8.85 / 9.08$ & $7.50 / 8.48 / 8.53$ & $6.69 / 5.25 / 4.67$ \\
\hline
\end{tabular}




\begin{tabular}{|c|c|c|c|c|c|}
\hline & Frec. de uso & Imaginación & Comprensión & Depresión & Ansiedad \\
\hline 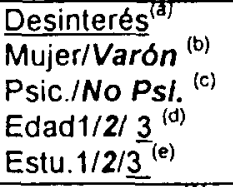 & \begin{tabular}{|l|}
$5.67(3.12)$ \\
$5.73 / 5.45$ \\
$6.21 / 5.19\left({ }^{*}\right)$ \\
$5.65 / 5.25 / 8.00$ \\
$5.92 / 5.42 / 6.08$ \\
\end{tabular} & $\begin{array}{l}6.37(3.28) \\
6.11 / 7.28\left(^{*}\right) \\
6.61 / 6.24 \\
6.49 / 6.44 / 4.88 \\
5.33 / 6.27 / 6.83 \\
\end{array}$ & $\begin{array}{l}8.70(2.22) \\
8.76 / 8.49 \\
9.19 / 8.27\left({ }^{*}\right) \\
8.74 / 8.56 / 8.38 \\
6.75 / 8.64 / 9.17 \\
\end{array}$ & \begin{tabular}{|l|}
$6.28(3.92)$ \\
$6.53 / 5.43$ \\
$7.77 / 4.96\left(^{*}\right)$ \\
$6.54 / 5.38 / 5.38$ \\
$5.25 / 5.82 / 7.52\left({ }^{*}\right)$ \\
\end{tabular} & \begin{tabular}{|l|}
$2.58(2.88)$ \\
$2.57 / 2.67$ \\
$2.80 / 2.43$ \\
$2.55 / 2.61 / 3.38$ \\
$2.85 / 2.60 / 2.52$ \\
\end{tabular} \\
\hline 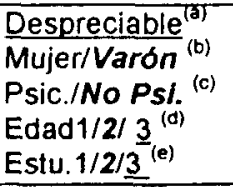 & \begin{tabular}{|l|}
$4.91(3.38)$ \\
$5.06 / 4.41$ \\
$5.78 / 4.18\left({ }^{*}\right)$ \\
$5.03 / 4.35 / 5.75$ \\
$5.08 / 4.47 / 5.80$ \\
\end{tabular} & $\begin{array}{l}6.14(3.37) \\
5.87 / 7.04\left(^{*}\right) \\
5.75 / 6.50 \\
6.12 / 6.29 / 6.00 \\
5.85 / 5.97 / 6.48 \\
\end{array}$ & $\begin{array}{l}8.77(2.18) \\
8.67 / 9.10 \\
9.11 / 8.47(*) \\
8.77 / 8.71 / 9.25 \\
8.08 / 8.60 / 9.29 \\
\end{array}$ & \begin{tabular}{|l|}
$5.37(3.70)$ \\
$5.41 / 5.24$ \\
$5.25 / 5.42$ \\
$5.47 / 4.71 / 6.63$ \\
$5.38 / 5.31 / 5.41$ \\
\end{tabular} & \begin{tabular}{|l|}
$3.39(3.45)$ \\
$3.40 / 3.43$ \\
$2.81 / 3.86\left({ }^{*}\right)$ \\
$3.38 / 3.27 / 3.63$ \\
$3.85 / 3.52 / 2.84$ \\
\end{tabular} \\
\hline 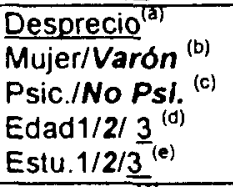 & \begin{tabular}{|l|}
$5.08(3.32)$ \\
$5.16 / 4.75$ \\
$5.73 / 4.48\left({ }^{*}\right)$ \\
$5.27 / 3.98 / 7.50(*)$ \\
$6.31 / 4.88 / 5.19$ \\
\end{tabular} & $\begin{array}{l}6.63(3.19) \\
6.48 / 7.12 \\
6.52 / 7.33 \\
6.53 / 6.98 / 6.88 \\
6.23 / 6.63 / 6.62 \\
\end{array}$ & $\begin{array}{l}8.84(2.03) \\
8.85 / 8.80 \\
9.08 / 8.62 \\
8.87 / 8.84 / 9.13 \\
8.77 / 8.74 / 9.05 \\
\end{array}$ & \begin{tabular}{|l|}
$7.53(2.89)$ \\
$7.41 / 7.90$ \\
$7.65 / 7.43$ \\
$7.77 / 6.84 / 7.38$ \\
$7.85 / 7.78 / 6.81$ \\
\end{tabular} & \begin{tabular}{|l|}
$5.05(3.72)$ \\
$5.06 / 5.10$ \\
$4.85 / 5.27$ \\
$5.12 / 5.12 / 4.25$ \\
$5.77 / 5.45 / 4.02(0)$ \\
\end{tabular} \\
\hline 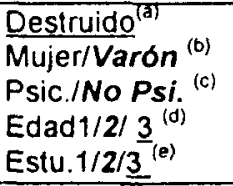 & \begin{tabular}{|l|}
$5.31(3.28)$ \\
$5.38 / 5.04$ \\
$5.81 / 4.88\left(^{*}\right)$ \\
$5.65 / 4.11 / 6.88$ \\
$6.33 / 5.24 / 5.31\left(^{*}\right)$ \\
\end{tabular} & \begin{tabular}{|l|}
$7.32(3.07)$ \\
$7.36 / 7.16$ \\
$7.65 / 7.05$ \\
$7.30 / 7.27 / 7.25$ \\
$6.00 / 7.30 / 7.57$ \\
\end{tabular} & $\begin{array}{l}8.64(2.25) \\
8.75 / 8.31 \\
9.08 / 8.26\left(^{\star}\right) \\
8.61 / 8.63 / 8.88 \\
8.08 / 8.47 / 9.10 \\
\end{array}$ & \begin{tabular}{|l|}
$6.67(3.31)$ \\
$6.84 / 6.65$ \\
$6.96 / 6.64$ \\
$6.85 / 6.56 / 6.50$ \\
$7.42 / 6.78 / 6.52$ \\
\end{tabular} & \begin{tabular}{|l|}
$4.87(3.53)$ \\
$5.01 / 4.53$ \\
$4.43 / 5.30$ \\
$5.01 / 4.58 / 4.88$ \\
$5.46 / 5.07 / 4.29$ \\
\end{tabular} \\
\hline 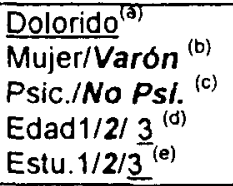 & \begin{tabular}{|l|}
$5.36(3.03)$ \\
$5.33 / 4.78$ \\
$5.65 / 5.12$ \\
$5.29 / 5.50 / 6.63$ \\
$6.23 / 5.09 / 5.86$ \\
\end{tabular} & \begin{tabular}{|l|}
$7.11(2.94)$ \\
$7.02 / 7.41$ \\
$7.18 / 7.10$ \\
$6.89 / 7.80 / 8.00$ \\
$7.15 / 6.97 / 7.43$ \\
\end{tabular} & $\begin{array}{l}8.94(1.75) \\
8.94 / 8.92 \\
9.14 / 8.76 \\
8.87 / 9.22 / 9.00 \\
8.77 / 8.88 / 9.07 \\
\end{array}$ & \begin{tabular}{|l|}
$5.76(3.31)$ \\
$5.77 / 5.84$ \\
$5.97 / 5.65$ \\
$5.97 / 5.31 / 5.63$ \\
$5.15 / 5.75 / 6.16$ \\
\end{tabular} & \begin{tabular}{|l|}
$4.36(3.27)$ \\
$4.33 / 4.51$ \\
$4.19 / 4.54$ \\
$4.58 / 4.04 / 3.63$ \\
$4.15 / 4.61 / 3.97$ \\
\end{tabular} \\
\hline $\begin{array}{l}\text { Dubitativo }^{(a)} \\
\text { Mujer/Varón }^{(b)} \\
\text { Psic./No Psi. }^{(\mathrm{c})} \\
\text { Edad1/2/ }^{(\mathrm{d})} \\
\text { Estu. } 1 / 2 / \mathbf{3}^{(\mathrm{e})}\end{array}$ & \begin{tabular}{|l|}
$4.26(3.23)$ \\
$4.35 / 3.98$ \\
$4.55 / 4.03$ \\
$4.31 / 4.17 / 4.13$ \\
$3.46 / 3.80 / 5.48(*)$ \\
\end{tabular} & \begin{tabular}{|l|}
$6.47(3.39)$ \\
$6.48 / 6.49$ \\
$6.79 / 6.27$ \\
$6.45 / 6.92 / 4.63$ \\
$5.85 / 6.10 / 7.46\left({ }^{*}\right)$ \\
\end{tabular} & $\begin{array}{l}8.45(2.37) \\
8.43 / 8.53 \\
9.02 / 7.96\left(^{*}\right) \\
8.48 / 8.76 / 7.00 \\
7.15 / 8.20 / 9.25(*) \\
\end{array}$ & \begin{tabular}{|l|}
$5.00(3.48)$ \\
$4.98 / 5.14$ \\
$5.52 / 4.58\left({ }^{*}\right)$ \\
$5.00 / 5.22 / 5.25$ \\
$5.31 / 4.66 / 5.86$ \\
\end{tabular} & \begin{tabular}{|l|}
$5.81(3.28)$ \\
$5.89 / 5.57$ \\
$5.88 / 5.77$ \\
$5.68 / 6.67 / 3.00\left({ }^{*}\right)$ \\
$6.38 / 5.57 / 6.41$ \\
\end{tabular} \\
\hline 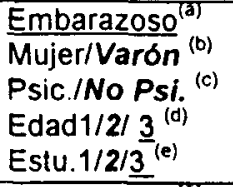 & \begin{tabular}{|l|}
$4.49(3.18)$ \\
$4.78 / 3.55\left({ }^{*}\right)$ \\
$4.67 / 4.35$ \\
$4.29 / 4.92 / 6.13$ \\
$4.92 / 4.31 / 4.81$ \\
\end{tabular} & \begin{tabular}{|l}
$6.28(3.31)$ \\
$6.30 / 6.27$ \\
$6.46 / 6.20$ \\
$6.15 / 6.92 / 6.63$ \\
$7.38 / 6.06 / 6.53$ \\
\end{tabular} & \begin{tabular}{|l|}
$8.64(2.03)$ \\
$8.71 / 8.41$ \\
$9.02 / 8.30\left(^{*}\right)$ \\
$8.47 / 9.16 / 8.88$ \\
$8.46 / 8.42 / 9.12$ \\
\end{tabular} & \begin{tabular}{|l|}
$3.95(3.21)$ \\
$4.03 / 3.76$ \\
$4.02 / 3.93$ \\
$3.82 / 4.12 / 5.75$ \\
$6.08 / 3.62 / 4.33(*)$ \\
\end{tabular} & \begin{tabular}{|l|}
$6.57(3.41)$ \\
$6.84 / 5.67\left(^{*}\right)$ \\
$7.25 / 5.97\left(^{*}\right)$ \\
$6.82 / 6.12 / 4.25$ \\
$6.23 / 6.56 / 6.78$ \\
\end{tabular} \\
\hline 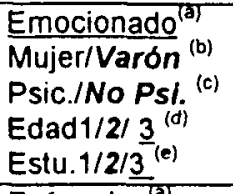 & \begin{tabular}{|l|}
$7.48(2.43)$ \\
$7.60 / 7.10$ \\
$7.71 / 7.30$ \\
$7.64 / 6.90 / 8.88\left({ }^{*}\right)$ \\
$8.08 / 7.34 / 7.66$ \\
\end{tabular} & \begin{tabular}{|l|}
$7.91(2.68)$ \\
$7.87 / 8.16$ \\
$7.84 / 8.06$ \\
$8.05 / 7.56 / 8.75$ \\
$7.46 / 8.01 / 7.77$ \\
\end{tabular} & \begin{tabular}{|l|}
$8.72(2.09)$ \\
$8.69 / 8.78$ \\
$8.89 / 8.55$ \\
$8.71 / 8.69 / 8.38$ \\
$7.77 / 8.74 / 8.81$ \\
\end{tabular} & \begin{tabular}{|l|}
$2.2582 .92)$ \\
$2.24 / 2.29$ \\
$2.69 / 1.83\left({ }^{*}\right)$ \\
$2.14 / 2.29 / 4.00$ \\
$2.62 / 1.85 / 3.09(*)$ \\
\end{tabular} & \begin{tabular}{|l|}
$5.81(3.46)$ \\
$5.74 / 6.06$ \\
$6.16 / 5.49$ \\
$6.15 / 4.67 / 5.75\left(^{*}\right)$ \\
$5.23 / 5.96 / 5.53$ \\
\end{tabular} \\
\hline 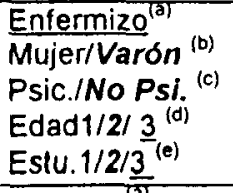 & \begin{tabular}{|l|}
$4.80(3.05)$ \\
$4.70 / 5.06$ \\
$5.06 / 4.54\left(^{*}\right)$ \\
$4.95 / 3.98 / 6.63\left({ }^{*}\right)$ \\
$5.00 / 4.73 / 4.85$ \\
\end{tabular} & \begin{tabular}{|l|}
$7.60(2.70)$ \\
$7.60 / 7.70$ \\
$7.64 / 7.61$ \\
$7.49 / 7.88 / 8.38$ \\
$8.31 / 7.40 / 7.67$ \\
\end{tabular} & \begin{tabular}{|l|}
$8.67(2.21)$ \\
$8.76 / 8.37$ \\
$9.02 / 8.36\left({ }^{*}\right)$ \\
$8.58 / 8.86 / 9.13$ \\
$8.38 / 8.51 / 9.05$ \\
\end{tabular} & \begin{tabular}{|l|}
$6.97(3.14)$ \\
$6.97 / 6.98$ \\
$6.75 / 7.18$ \\
$6.87 / 7.20 / 7.50$ \\
$7.00 / 7.07 / 6.71$ \\
\end{tabular} & \begin{tabular}{|l|}
$4.98(3.46)$ \\
$5.10 / 4.65$ \\
$4.60 / 5.28$ \\
$5.00 / 5.16 / 4.13$ \\
$5.85 / 5.37 / 3.83(*)$ \\
\end{tabular} \\
\hline 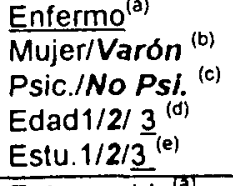 & \begin{tabular}{|l|}
$7.21(2.94)$ \\
$7.49 / 6.27\left({ }^{*}\right)$ \\
$7.88 / 6.65\left(^{*}\right)$ \\
$7.34 / 6.69 / 8.25$ \\
$7.69 / 6.96 / 7.76$ \\
\end{tabular} & \begin{tabular}{|l|}
$8.57(2.41)$ \\
$8.64 / 8.33$ \\
$8.93 / 8.27\left(^{*}\right)$ \\
$8.62 / 8.41 / 9.25$ \\
$8.15 / 8.57 / 8.64$ \\
\end{tabular} & \begin{tabular}{|l|}
$9.15(1.18)$ \\
$9.22 / 8.90$ \\
$9.33 / 9.05$ \\
$9.07 / 9.51 / 9.25$ \\
$8.77 / 9.15 / 9.32$ \\
\end{tabular} & \begin{tabular}{|l|}
$7.83(2.73)$ \\
$7.88 / 7.71$ \\
$7.82 / 7.85$ \\
$7.80 / 7.84 / 8.50$ \\
$8.54 / 7.77 / 7.83$ \\
\end{tabular} & \begin{tabular}{|l|}
$6.11(3.50)$ \\
$6.16 / 5.96$ \\
$6.42 / 5.84$ \\
$6.08 / 6.25 / 6.88$ \\
$7.08 / 5.92 / 6.54$ \\
\end{tabular} \\
\hline 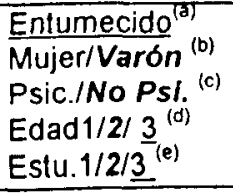 & \begin{tabular}{|l|}
$2.19(2.63)$ \\
$2.18 / 2.22$ \\
$2.20 / 2.19$ \\
$1.97 / 2.67 / 3.75$ \\
$3.15 / 2.01 / 2.36$ \\
\end{tabular} & \begin{tabular}{|l|}
$4.60(3.35)$ \\
$4.52 / 4.82$ \\
$4.80 / 4.43$ \\
$4.18 / 5.67 / 6.13\left({ }^{*}\right)$ \\
$5.38 / 4.28 / 5.00$ \\
\end{tabular} & \begin{tabular}{l|}
$6.34(3.32)$ \\
$6.23 / 6.69$ \\
$6.32 / 6.32$ \\
$5.62 / 7.98 / 8.88\left({ }^{*}\right)$ \\
$7.08 / 5.94 / 6.92$ \\
\end{tabular} & \begin{tabular}{|l}
$4.66(3.45)$ \\
$4.65 / 4.71$ \\
$5.55 / 3.96\left({ }^{*}\right)$ \\
$4.77 / 4.20 / 5.00$ \\
$5.54 / 4.49 / 4.93$ \\
\end{tabular} & \begin{tabular}{|l|}
$3.49(3.01)$ \\
$3.57 / 3.25$ \\
$3.71 / 3.27$ \\
$3.41 / 3.80 / 1.75$ \\
$4.69 / 3.22 / 3.84$ \\
\end{tabular} \\
\hline
\end{tabular}




\begin{tabular}{|c|c|c|c|c|c|}
\hline & Frec de uso & Imaginación & Comprensión & Laposion & Ansiedad \\
\hline 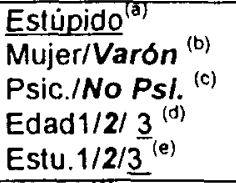 & $\begin{array}{l}5.83(3.36) \\
6.00 / 5.29 \\
6.72 / 5.09\left(^{*}\right) \\
6.12 / 5.12 / 5.38 \\
5.92 / 5.57 / 6.39 \\
\end{array}$ & $\begin{array}{l}5.89(3.41) \\
5.69 / 6.55 \\
5.84 / 5.96 \\
5.80 / 6.60 / 4.75 \\
6.00 / 5.55 / 6.56\end{array}$ & $\begin{array}{l}8.46(2.38) \\
8.54 / 8.25 \\
8.77 / 8.20 \\
8.50 / 8.43 / 8.88 \\
7.85 / 8.38 / 8.71\end{array}$ & $\begin{array}{l}3.18(3.36) \\
3.23 / 3.08 \\
3.36 / 3.06 \\
3.36 / 2.76 / 2.38 \\
2.54 / 3.12 / 3.50\end{array}$ & $\begin{array}{l}2.58(2.90) \\
2.48 / 2.90 \\
2.56 / 2.62 \\
2.69 / 2.10 / 4.25 \\
3.31 / 2.76 / 2.12\end{array}$ \\
\hline 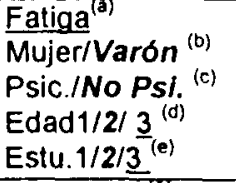 & $\begin{array}{l}5.96(3.15) \\
6.00 / 5.78 \\
6.48 / 5.49\left(^{\star}\right) \\
6.01 / 5.69 / \underline{6.75} \\
5.85 / 5.64 / 6.68 \\
\end{array}$ & $\begin{array}{l}7.39(2.92) \\
7.31 / 7.72 \\
7.78 / 7.09 \\
7.49 / 7.35 / 6.75 \\
6.69 / 7.27 / 7.72\end{array}$ & $\begin{array}{l}8.76(2.03) \\
8.88 / 8.37 \\
9.09 / 8.46\left(^{\star}\right) \\
8.72 / 8.78 / 9.00 \\
8.00 / 8.65 / 9.12 \\
\end{array}$ & \begin{tabular}{|l|}
$6.21(3.53)$ \\
$6.31 / 5.86$ \\
$7.35 / 5.19\left(^{*}\right)$ \\
$6.14 / 6.35 / 5.13$ \\
$6.31 / 5.64 / 7.38\left({ }^{*}\right)$ \\
\end{tabular} & \begin{tabular}{|l|}
$4.87(3.48)$ \\
$5.16 / 4.04$ \\
$5.22 / 4.60$ \\
$4.77 / 5.61 / 4.00$ \\
$5.85 / 4.64 / 5.26$ \\
\end{tabular} \\
\hline $\begin{array}{l}\text { Fracasado }^{(a)} \\
\text { Mujer/Varón }^{\text {(b) }} \\
\text { Psic./No Psi. }^{\text {(c) }} \\
\text { Edad } 1 / 2 / 3^{\text {(d) }} \\
{\text { Estu. } 1 / 2 / 3^{(\mathrm{e})}}^{{ }^{2}}\end{array}$ & \begin{tabular}{|l|}
$5.73(3.61)$ \\
$5.90 / 5.14$ \\
$6.39 / 5.18\left(^{*}\right)$ \\
$5.99 / 4.69 / 6.63\left(^{*}\right)$ \\
$4.92 / 5.64 / 6.03$ \\
\end{tabular} & $\begin{array}{l}7.07(3.12) \\
7.01 / 7.27 \\
7.17 / 6.96 \\
6.97 / 7.25 / 7.63 \\
7.85 / 6.79 / 7.45 \\
\end{array}$ & $\begin{array}{l}9.11(2.12) \\
9.05 / 9.30 \\
9.40 / 8.86 \\
9.14 / 9.13 / 9.38 \\
9.00 / 9.07 / 9.20 \\
\end{array}$ & $\begin{array}{l}8.71(2.32) \\
8.81 / 8.39 \\
8.99 / 8.46 \\
8.77 / 8.41 / 9.38 \\
8.31 / 8.82 / 8.56 \\
\end{array}$ & \begin{tabular}{|l|}
$5.78(3.65)$ \\
$5.82 / 5.76$ \\
$5.30 / 6.20$ \\
$5.78 / 5.63 / 7.50$ \\
$6.69 / 6.01 / 4.95$ \\
\end{tabular} \\
\hline 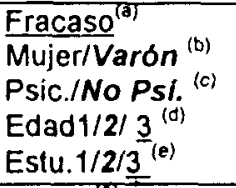 & \begin{tabular}{|l|}
$6.09(3.27)$ \\
$6.16 / 5.80$ \\
$6.69 / 5.56\left(^{*}\right)$ \\
$6.35 / 5.02 / 7.50\left(^{*}\right)$ \\
$7.31 / 5.93 / 6.17$ \\
\end{tabular} & $\begin{array}{l}6.85(3.16) \\
6.77 / 7.18 \\
6.89 / 6.84 \\
6.79 / 7.10 / 6.75 \\
6.15 / 6.82 / 6.97 \\
\end{array}$ & $\begin{array}{l}9.00(1.93) \\
8.99 / 9.02 \\
9.24 / 8.79 \\
9.02 / 8.94 / 9.00 \\
9.08 / 8.88 / 9.22 \\
\end{array}$ & \begin{tabular}{|l|}
$8.18(2.71)$ \\
$8.17 / 8.22$ \\
$8.33 / 8.04$ \\
$8.44 / 7.37 / 8.13$ \\
$8.00 / 8.27 / 7.91$ \\
\end{tabular} & \begin{tabular}{|l|}
$5.74(3.74)$ \\
$5.83 / 5.53$ \\
$5.27 / 6.19$ \\
$5.77 / 5.94 / 4.50\left(^{*}\right)$ \\
$5.46 / 6.28 / 4.53\left(^{*}\right)$ \\
\end{tabular} \\
\hline $\begin{array}{l}\text { Herido }^{(d)} \\
\text { Mujer/Varón }^{\text {(b) }} \\
\text { Psic./No Psi. } \\
\text { Edad } 1 / 2 / 3^{\text {(d) }} \\
{\text { Estu. } 1 / 2 / 3^{\text {(e) }}}\end{array}$ & $\begin{array}{l}6.37(3.19) \\
6.66 / 5.42\left(^{\wedge}\right) \\
6.80 / 6.04 \\
6.47 / 6.17 / 6.75 \\
6.00 / 6.09 / 7.17 \\
\end{array}$ & $\begin{array}{l}8.34(2.63) \\
8.32 / 8.35 \\
8.66 / 8.05 \\
8.42 / 7.94 / 9.75 \\
8.00 / 8.34 / 8.38 \\
\end{array}$ & $\begin{array}{l}9.26(1.75) \\
9.22 / 9.39 \\
9.51 / 9.04\left(^{\star}\right) \\
9.30 / 9.20 / 9.88 \\
9.31 / 9.25 / 9.27 \\
\end{array}$ & $\begin{array}{l}5.36(3.44) \\
5.54 / 4.78 \\
5.50 / 5.27 \\
5.50 / 4.61 / 7.63\left({ }^{*}\right) \\
5.00 / 5.62 / 4.91 \\
\end{array}$ & $\begin{array}{l}4.81(3.59) \\
4.94 / 4.49 \\
4.95 / 4.73 \\
5.19 / 4.27 / 2.75 \\
2.69 / 5.15 / 4.64\left(^{*}\right) \\
\end{array}$ \\
\hline 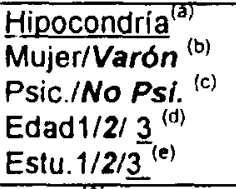 & \begin{tabular}{|l|}
$3.05(2.91)$ \\
$2.99 / 3.20$ \\
$3.85 / 2.33\left({ }^{\star}\right)$ \\
$2.97 / 3.12 / 4.00$ \\
$1.46 / 2.98 / 3.47$ \\
\end{tabular} & $\begin{array}{l}5.43(3.79) \\
5.40 / 5.49 \\
5.54 / 5.27 \\
5.05 / 6.57 / 4.88\left(^{*}\right) \\
5.85 / 5.01 / 6.16\end{array}$ & \begin{tabular}{|l|}
$6.73(3.49)$ \\
$6.64 / 6.95$ \\
$7.41 / 6.11\left(^{*}\right)$ \\
$6.27 / 7.96 / 7.25\left(^{*}\right)$ \\
$5.92 / 6.47 / 7.35\left({ }^{*}\right)$ \\
\end{tabular} & \begin{tabular}{|l|}
$5.31(3.32)$ \\
$5.35 / 5.59$ \\
$4.93 / 5.83\left({ }^{*}\right)$ \\
$5.28 / 6.10 / 5.38$ \\
$4.46 / 5.53 / 5.46$ \\
\end{tabular} & $\begin{array}{l}7.32(3.18) \\
7.49 / 6.76 \\
8.01 / 6.69\left({ }^{*}\right) \\
7.60 / 6.77 / 6.88 \\
5.38 / 7.36 / 7.72( \\
\end{array}$ \\
\hline 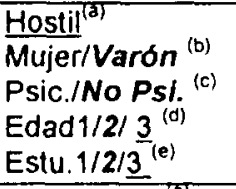 & \begin{tabular}{|l|}
$3.60(2.94)$ \\
$3.48 / 3.88$ \\
$4.42 / 2.86\left(^{\star}\right)$ \\
$3.65 / 3.57 / 1.88$ \\
$1.69 / 3.38 / 4.47\left({ }^{*}\right)$ \\
\end{tabular} & \begin{tabular}{|l}
$6.40(3.24)$ \\
$6.31 / 6.62$ \\
$6.53 / 6.26$ \\
$6.18 / 6.90 / 6.38$ \\
$6.00 / 6.29 / 6.67$ \\
\end{tabular} & \begin{tabular}{|l|}
$8.33(2.52)$ \\
$8.31 / 8.38$ \\
$8.94 / 7.80\left(^{\star}\right)$ \\
$8.17 / 8.78 / 9.14$ \\
$7.33 / 8.22 / 8.76$ \\
\end{tabular} & \begin{tabular}{|l|}
$3.90(3.30)$ \\
$4.01 / 3.48$ \\
$4.07 / 3.72$ \\
$3.80 / 4.14 / 5.00$ \\
$5.08 / 3.58 / 4.53$ \\
\end{tabular} & \begin{tabular}{|l}
$5.25(3.55)$ \\
$5.33 / 5.12$ \\
$6.54 / 4.20\left(^{*}\right)$ \\
$5.47 / 5.00 / 3.75$ \\
$4.15 / 5.14 / 5.88$ \\
\end{tabular} \\
\hline 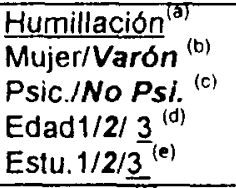 & $\begin{array}{l}4.74(3.33) \\
4.81 / 4.49 \\
5.27 / 4.30\left(^{*}\right) \\
4.90 / 3.98 / 6.50 \\
6.00 / 4.53 / 4.86 \\
\end{array}$ & \begin{tabular}{|l}
$6.77(3.07)$ \\
$6.58 / 7.50$ \\
$6.95 / 6.70$ \\
$6.82 / 6.67 / 7.63$ \\
$6.15 / 6.86 / 6.66$ \\
\end{tabular} & \begin{tabular}{|l|}
$8.73(2.05)$ \\
$8.74 / 8.67$ \\
$9.04 / 8.45\left(^{*}\right)$ \\
$8.69 / 8.86 / 8.50$ \\
$8.15 / 8.61 / 9.07$ \\
\end{tabular} & \begin{tabular}{|l|}
$6.89(3.23)$ \\
$6.79 / 7.29$ \\
$6.65 / 7.14$ \\
$6.96 / 6.82 / 6.13$ \\
$6.46 / 7.10 / 6.45$ \\
\end{tabular} & $\begin{array}{l}4.84(3.59) \\
4.84 / 4.74 \\
4.43 / 5.15 \\
4.94 / 4.51 / 4.88 \\
6.08 / 5.01 / 4.19 \\
\end{array}$ \\
\hline 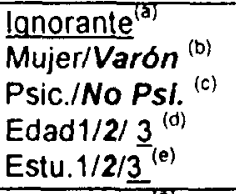 & \begin{tabular}{|l|}
$5.69(3.46)$ \\
$5.75 / 5.44$ \\
$6.03 / 5.37\left(^{*}\right)$ \\
$5.88 / 4.73 / 8.00\left({ }^{*}\right)$ \\
$7.08 / 5.63 / 5.48$ \\
\end{tabular} & \begin{tabular}{|l|}
$6.00(3.52)$ \\
$5.80 / 5.76$ \\
$5.81 / 6.22$ \\
$6.03 / 6.48 / 4.50$ \\
$5.77 / 5.99 / 6.00$ \\
\end{tabular} & \begin{tabular}{|l|}
$8.79(2.03)$ \\
$8.86 / 8.58$ \\
$9.08 / 8.53\left(^{*}\right)$ \\
$8.76 / 8.98 / 8.88$ \\
$8.15 / 8.73 / 9.03$ \\
\end{tabular} & $\begin{array}{l}3.01(3.40) \\
2.79 / 3.76 \\
2.54 / 3.46(") \\
3.17 / 2.38 / 4.13 \\
3.92 / 3.31 / 2.11\left(^{*}\right) \\
\end{array}$ & $\begin{array}{l}2.38(2.99) \\
2.23 / 2.86 \\
2.07 / 2.66 \\
2.42 / 2.08 / 3.88 \\
4.38 / 2.53 / 1.54(\end{array}$ \\
\hline $\begin{array}{l}\text { Imperfecto }^{(\mathrm{a})} \\
\text { Mujer/Varón }^{\text {(b) }} \\
\text { Psic./No Psl. }^{\text {(c) }} \\
\text { Edad } 1 / 2 / \underline{3}^{\text {(d) }} \\
{\text { Estu. } 1 / 2 / \underline{3}^{\text {(e) }}}^{{ }^{2}}\end{array}$ & \begin{tabular}{|l|}
$4.94(3.34)$ \\
$5.01 / 4.67$ \\
$5.33 / 4.57$ \\
$5.21 / 3.88 / 6.00\left(^{*}\right)$ \\
$5.08 / 4.82 / 5.10$ \\
\end{tabular} & \begin{tabular}{|l|}
$6.10(3.37)$ \\
$5.99 / 6.54$ \\
$5.97 / 6.23$ \\
$6.99 / 6.57 / 6.50$ \\
$6.77 / 5.94 / 6.19$ \\
\end{tabular} & \begin{tabular}{|l}
$8.65(2.23)$ \\
$8.64 / 8.69$ \\
$8.88 / 8.45$ \\
$8.66 / 8.80 / 8.63$ \\
$8.31 / 8.61 / 8.78$ \\
\end{tabular} & $\begin{array}{l}4.46(3.57) \\
4.14 / 5.51\left(^{*}\right) \\
4.69 / 4.26 \\
4.58 / 4.08 / 4.38 \\
4.08 / 4.43 / 4.47 \\
\end{array}$ & \begin{tabular}{|l|}
$3.91(3.52)$ \\
$3.81 / 4.29$ \\
$3.86 / 3.97$ \\
$3.81 / 4.20 / 5.25$ \\
$5.46 / 3.94 / 3.52$ \\
\end{tabular} \\
\hline $\begin{array}{l}\text { Inadecuado }^{(d)} \\
\text { Mujer/Varón }^{\text {(b) }} \\
\text { Psic./No Psi.j }^{\text {(c) }} \\
{\text { Edad } 1 / 2 / 3^{\text {(d) }}}^{\text {(e) }} \\
{\text { Estu. } 1 / 2 / \underline{3}^{\text {(e) }}}\end{array}$ & $\begin{array}{l}4.84(3.22) \\
4.79 / 4.88 \\
5.45 / 4.28\left(^{\star}\right) \\
5.07 / 3.90 / 5.88 \\
4.46 / 4.72 / 5.12 \\
\end{array}$ & \begin{tabular}{|l|}
$5.33(3.48)$ \\
$5.04 / 6.34\left(^{*}\right)$ \\
$5.06 / 5.63$ \\
$5.28 / 5.71 / 5.63$ \\
$4.92 / 5.38 / 5.28$ \\
\end{tabular} & \begin{tabular}{|l}
$8.15(2.48)$ \\
$8.32 / 7.63$ \\
$8.63 / 7.74\left(^{\star}\right)$ \\
$8.14 / 8.29 / 8.25$ \\
$\left.6.08 / 8.12 / 8.61{ }^{*}\right)$ \\
\end{tabular} & \begin{tabular}{|l}
$3.44(3.31)$ \\
$3.24 / 4.14$ \\
$3.69 / 3.25$ \\
$3.44 / 3.20 / 4.63$ \\
$4.31 / 3.23 / 3.55$ \\
\end{tabular} & $\begin{array}{l}3.32(3.23) \\
3.22 / 3.55\left(^{\star}\right) \\
3.56 / 3.10 \\
3.36 / 3.12 / 3.75 \\
3.69 / 3.27 / 3.38 \\
\end{array}$ \\
\hline
\end{tabular}




\begin{tabular}{|c|c|c|c|c|c|}
\hline & Frec. de uso & Imaginación & Comprensión & Depresión & Ansiedad \\
\hline 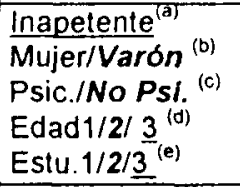 & \begin{tabular}{|l|}
$2.90(3.20)$ \\
$2.96 / 2.75$ \\
$2.85 / 2.97$ \\
$2.74 / 3.47 / 3.75$ \\
$3.54 / 2.75 / 3.05$ \\
\end{tabular} & \begin{tabular}{|l|}
$5.38(3.70)$ \\
$5.48 / 5.18$ \\
$5.81 / 5.11$ \\
$5.13 / 6.38 / 6.00$ \\
$5.54 / 5.08 / 6.02$ \\
\end{tabular} & \begin{tabular}{|l|}
$3.71(3.28)$ \\
$7.45 / 6.96$ \\
$7.49 / 7.17$ \\
$6.91 / 8.38 / 8.63\left({ }^{*}\right)$ \\
$6.54 / 7.13 / 7.76$ \\
\end{tabular} & \begin{tabular}{|l|}
$6.54(3.70)$ \\
$6.78 / 5.76$ \\
$7.94 / 5.31\left({ }^{*}\right)$ \\
$6.54 / 6.55 / 5.38$ \\
$4.77 / 6.29 / 7.55\left({ }^{*}\right)$ \\
\end{tabular} & \begin{tabular}{|l|}
$2.93(3.20)$ \\
$3.04 / 2.63$ \\
$3.04 / 2.88$ \\
$2.78 / 3.49 / 2.13$ \\
$2.85 / 2.96 / 3.02$ \\
\end{tabular} \\
\hline 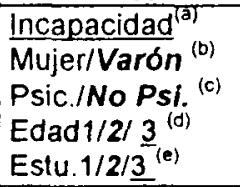 & $\begin{array}{l}4.90(3.31) \\
5.13 / 4.12 \\
5.27 / 4.59 \\
4.71 / 5.31 / 6.38 \\
5.62 / 4.58 / 5.59 \\
\end{array}$ & \begin{tabular}{|l|}
$7.36(2.99)$ \\
$7.41 / 7.22$ \\
$7.30 / 7.46$ \\
$7.30 / 7.52 / 8.63$ \\
$7.00 / 7.54 / 7.05$ \\
\end{tabular} & \begin{tabular}{|l|}
$8.92(1.90)$ \\
$8.96 / 8.76$ \\
$8.98 / 8.85$ \\
$8.83 / 9.10 / 9.25$ \\
$8.77 / 8.88 / 9.02$ \\
\end{tabular} & \begin{tabular}{|l|}
$7.18(3.16)$ \\
$7.33 / 6.71$ \\
$7.48 / 6.90$ \\
$7.05 / 7.22 / 8.50$ \\
$5.46 / 7.40 / 7.07$ \\
\end{tabular} & \begin{tabular}{|l|}
$5.80(3.64)$ \\
$5.77 / 5.94$ \\
$5.52 / 6.05$ \\
$5.83 / 5.80 / 5.75$ \\
$4.77 / 6.20 / 5.14$ \\
\end{tabular} \\
\hline 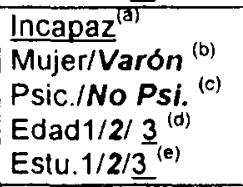 & \begin{tabular}{|l|}
$5.91(3.16)$ \\
$6.13 / 5.16\left(^{*}\right)$ \\
$6.38 / 5.49\left(^{*}\right)$ \\
$6.12 / 5.24 / 6.14$ \\
$5.85 / 5.75 / 6.25$ \\
\end{tabular} & \begin{tabular}{|l|}
$6.36(3.24)$ \\
$6.40 / 6.33$ \\
$6.67 / 6.21$ \\
$6.40 / 6.69 / 4.88$ \\
$5.85 / 6.20 / 6.74$ \\
\end{tabular} & $\begin{array}{l}8.70(2.12) \\
8.82 / 8.29 \\
9.01 / 8.42\left({ }^{*}\right) \\
8.75 / 8.65 / 8.63 \\
7.85 / 8.61 / 9.03 \\
\end{array}$ & \begin{tabular}{|l|}
$6.22(3.45)$ \\
$6.07 / 6.78$ \\
$6.49 / 6.00$ \\
$6.38 / 5.59 / 7.13$ \\
$6.00 / 6.26 / 6.07$ \\
\end{tabular} & \begin{tabular}{|l|}
$4.87(3.53)$ \\
$4.09 / 4.88$ \\
$4.76 / 5.02$ \\
$4.87 / 4.96 / 4.63$ \\
$5.85 / 5.08 / 4.10\left({ }^{*}\right)$ \\
\end{tabular} \\
\hline 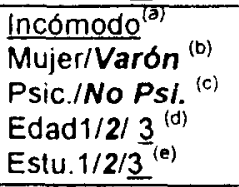 & \begin{tabular}{|l|}
$6.83(2.78)$ \\
$7.05 / 6.10\left(^{*}\right)$ \\
$7.40 / 6.53\left({ }^{*}\right)$ \\
$6.99 / 6.39 / 7.25$ \\
$6.46 / 6.80 / 6.93$ \\
\end{tabular} & \begin{tabular}{|l|}
$7.00(2.99)$ \\
$6.90 / 7.35$ \\
$7.24 / 6.81$ \\
$7.01 / 7.14 / 7.00$ \\
$6.23 / 6.93 / 7.28$ \\
\end{tabular} & $\begin{array}{l}8.70(2.01) \\
8.74 / 8.57 \\
9.21 / 8.26\left({ }^{*}\right) \\
8.69 / 8.82 / 8.25 \\
7.92 / 8.51 / 9.25(*) \\
\end{array}$ & \begin{tabular}{|l|}
$4.09(3.45)$ \\
$4.15 / 3.96$ \\
$4.77 / 3.55\left(^{*}\right)$ \\
$4.36 / 3.61 / 2.88$ \\
$2.69 / 4.09 / 4.57$ \\
\end{tabular} & \begin{tabular}{|l|}
$6.43(3.43)$ \\
$6.60 / 5.84$ \\
$7.26 / 5.68\left(^{*}\right)$ \\
$6.75 / 5.37 / 5.63\left(^{*}\right)$ \\
$5.31 / 6.51 / 6.52\left({ }^{*}\right)$ \\
\end{tabular} \\
\hline $\begin{array}{l}\text { Incompetente }^{(\mathrm{a})} \\
\text { Mujer/Varón }^{(\mathrm{b})} \\
\text { Psic./No Psi. }^{(\mathrm{c})} \\
\text { Edad1/2/ }^{(\mathrm{d})} \\
\text { Estu.1/2/3 }^{(\mathrm{e})}\end{array}$ & $\begin{array}{l}5.33(3.26) \\
5.43 / 5.02 \\
6.13 / 4.65\left(^{*}\right) \\
5.63 / 4.29 / 5.75\left(^{*}\right) \\
4.15 / 5.30 / 5.56 \\
\end{array}$ & \begin{tabular}{|l|}
$6.51(3.35)$ \\
$6.49 / 6.68$ \\
$6.51 / 6.55$ \\
$6.50 / 6.57 / 7.25$ \\
$6.15 / 6.65 / 6.19$ \\
\end{tabular} & $\begin{array}{l}8.53(2.29) \\
8.58 / 8.35 \\
9.02 / 8.10\left(^{*}\right) \\
8.54 / 8.47 / 8.38 \\
6.46 / 8.50 / 8.98\left({ }^{*}\right)\end{array}$ & \begin{tabular}{|l|}
$5.30(3.73)$ \\
$5.33 / 5.27$ \\
$5.57 / 5.09$ \\
$5.50 / 4.55 / 5.75$ \\
$4.54 / 5.54 / 4.88$ \\
\end{tabular} & $\begin{array}{l}4.34(3.69) \\
4.36 / 4.31 \\
4.01 / 4.66 \\
4.26 / 4.24 / 7.00 \\
5.46 / 4.67 / 3.17\left({ }^{\circ}\right) \\
\end{array}$ \\
\hline 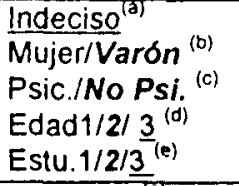 & \begin{tabular}{|l|}
$6.33(2.90)$ \\
$6.60 / 5.43\left(^{*}\right)$ \\
$6.81 / 5.91\left(^{*}\right)$ \\
$6.47 / 5.63 / 7.88$ \\
$5.92 / 6.14 / 6.78$ \\
\end{tabular} & \begin{tabular}{|l}
$6.97(3.00)$ \\
$6.84 / 7.44$ \\
$6.99 / 7.01$ \\
$7.04 / 7.25 / 5.38$ \\
$6.67 / 6.96 / 7.09$ \\
\end{tabular} & $\begin{array}{l}8.73(1.97) \\
8.77 / 8.61 \\
9.06 / 8.43\left(^{*}\right) \\
8.80 / 8.54 / 8.13 \\
6.75 / 8.70 / 9.14\left(^{*}\right) \\
\end{array}$ & \begin{tabular}{|l|l|}
$5.79(3.42)$ \\
$5.69 / 6.08$ \\
$6.38 / 5.31\left(^{*}\right)$ \\
$6.03 / 5.27 / 4.88$ \\
$5.00 / 5.64 / 6.38$ \\
\end{tabular} & \begin{tabular}{|l|}
$6.24(3.43)$ \\
$6.20 / 6.29$ \\
$6.40 / 6.06$ \\
$6.20 / 6.53 / 3.88$ \\
$5.00 / 6.49 / 5.79$ \\
\end{tabular} \\
\hline 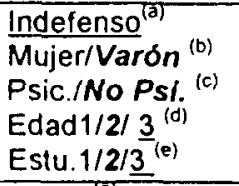 & \begin{tabular}{|l|}
$5.51(3.04)$ \\
$5.59 / 5.20$ \\
$6.08 / 4.98$ \\
$5.58 / 4.85 / 7.50$ \\
$6.50 / 5.27 / 5.80$ \\
\end{tabular} & \begin{tabular}{|l|}
$7.0583 .08)$ \\
$7.06 / 7.12$ \\
$7.08 / 7.05$ \\
$7.07 / 6.98 / 7.50$ \\
$6.75 / 6.95 / 7.24$ \\
\end{tabular} & \begin{tabular}{|l|}
$8.86(1.89)$ \\
$8.94 / 8.59$ \\
$9.08 / 8.65$ \\
$8.77 / 9.04 / 9.00$ \\
$9.00 / 8.67 / 9.20$ \\
\end{tabular} & \begin{tabular}{|l|}
$6.70(3.10)$ \\
$6.59 / 7.10$ \\
$6.78 / 6.66$ \\
$6.94 / 5.94 / 7.38$ \\
$6.33 / 6.80 / 6.47$ \\
\end{tabular} & \begin{tabular}{|l|}
$5.56(3.60)$ \\
$5.55 / 5.69$ \\
$5.77 / 5.41$ \\
$5.84 / 5.10 / 4.13$ \\
$4.54 / 5.53 / 5.90$ \\
\end{tabular} \\
\hline 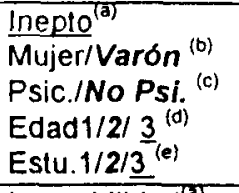 & \begin{tabular}{|l|}
$3.54(3.33)$ \\
$3.33 / 4.14$ \\
$3.98 / 3.11$ \\
$3.44 / 3.51 / 4.86$ \\
$3.46 / 3.33 / 3.93$ \\
\end{tabular} & \begin{tabular}{|l|}
$5.21(3.51)$ \\
$4.90 / 6.16\left(^{*}\right)$ \\
$5.33 / 5.08$ \\
$4.99 / 6.10 / 4.25$ \\
$5.17 / 5.01 / 5.51$ \\
\end{tabular} & \begin{tabular}{|l|}
$7.31(3.12)$ \\
$7.26 / 7.47$ \\
$7.85 / 6.82\left(^{*}\right)$ \\
$7.01 / 7.92 / 8.50$ \\
$6.15 / 7.01 / 8.14\left(^{*}\right)$ \\
\end{tabular} & \begin{tabular}{|l|}
$3.83(3.61)$ \\
$3.75 / 4.18$ \\
$3.69 / 4.02$ \\
$3.77 / 3.73 / 6.13$ \\
$2.77 / 3.99 / 3.69$ \\
\end{tabular} & \begin{tabular}{|l|}
$2.95(3.23)$ \\
$2.88 / 3.24$ \\
$2.66 / 3.25$ \\
$2.92 / 2.94 / 4.00$ \\
$2.92 / 3.25 / 2.26$ \\
\end{tabular} \\
\hline 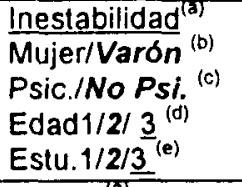 & \begin{tabular}{|l|}
$4.82(3.07)$ \\
$5.01 / 4.20$ \\
$5.22 / 4.49$ \\
$4.81 / 4.73 / 6.25$ \\
$4.54 / 4.71 / 5.17$ \\
\end{tabular} & \begin{tabular}{|l|}
$6.12(3.26)$ \\
$6.14 / 6.08$ \\
$6.00 / 6.22$ \\
$5.90 / 6.94 / 5.88$ \\
$5.31 / 6.09 / 6.28$ \\
\end{tabular} & \begin{tabular}{|l|}
$8.67(2.02)$ \\
$8.78 / 8.35$ \\
$8.85 / 8.51$ \\
$8.49 / 9.33 / 8.13\left({ }^{*}\right)$ \\
$7.54 / 8.54 / 9.19\left({ }^{*}\right)$ \\
\end{tabular} & \begin{tabular}{|l}
$7.40(2.94)$ \\
$7.33 / 7.57$ \\
$7.23 / 7.56$ \\
$7.51 / 7.39 / 6.13$ \\
$6.59 / 7.57 / 7.29$ \\
\end{tabular} & \begin{tabular}{|l|}
$7.79(2.60)$ \\
$7.91 / 7.39$ \\
$7.83 / 7.73$ \\
$7.74 / 8.08 / 6.25$ \\
$7.00 / 8.05 / 7.31$ \\
\end{tabular} \\
\hline $\begin{array}{l}\text { Inferior }{ }^{(2)} \\
\text { Mujer/Varón } \\
\text { Psic./No Psi }^{\left({ }^{(c)}\right.} \\
{\text { Edad } 1 / 2 / \underline{3}^{(d)}}^{(d)} \\
\text { Estu. } 1 / 2 / 3^{(e)}\end{array}$ & \begin{tabular}{|l|}
$5.99(3.34)$ \\
$5.40 / 4.84$ \\
$6.19 / 4.50$ \\
$5.70 / 4.06 / 5.14$ \\
$4.69 / 5.20 / 5.64$ \\
\end{tabular}$\left(^{*}\right)$ & \begin{tabular}{|l|}
$5.73(3.59)$ \\
$5.57 / 6.27$ \\
$6.00 / 5.52$ \\
$5.81 / 5.83 / 4.75$ \\
$4.50 / 5.72 / 5.89$ \\
\end{tabular} & \begin{tabular}{|l|}
$8.49(2.32)$ \\
$8.59 / 8.16$ \\
$8.68 / 8.31$ \\
$8.52 / 8.41 / 9.00$ \\
$8.08 / 8.34 / 8.86$ \\
\end{tabular} & 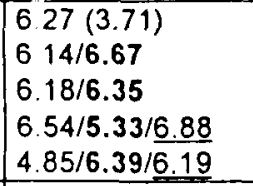 & \begin{tabular}{|l|}
$3.84(3.53)$ \\
$3.89 / 3.71$ \\
$3.72 / 3.96$ \\
$3.91 / 3.49 / 4.13$ \\
$4.08 / 3.93 / 3.55$ \\
\end{tabular} \\
\hline 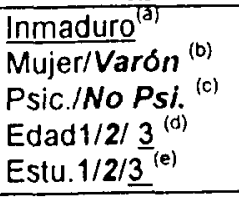 & \begin{tabular}{|l|}
$5.31(3.15)$ \\
$5.43 / 5.00$ \\
$6.08 / 4.69\left({ }^{*}\right)$ \\
$5.53 / 4.45 / 6.25$ \\
$4.69 / 5.20 / 5.62$ \\
\end{tabular} & \begin{tabular}{|l|}
$6.19(3.23)$ \\
$6.16 / 6.41$ \\
$6.48 / 6.04$ \\
$6.05 / 6.88 / 5.88$ \\
$6.31 / 6.05 / 6.53$ \\
\end{tabular} & \begin{tabular}{|l|}
$8.58(2.11)$ \\
$8.64 / 8.41$ \\
$9.10 / 8.13\left(^{*}\right)$ \\
$8.56 / 8.57 / 9.25$ \\
$8.08 / 8.35 / 9.19(*)$ \\
\end{tabular} & \begin{tabular}{|l|}
$2.70(2.88)$ \\
$2.58 / 3.14$ \\
$2.79 / 2.65$ \\
$2.70 / 2.63 / 4.13$ \\
$2.85 / 2.79 / 2.45$ \\
\end{tabular} & \begin{tabular}{|l|}
$3.21(3.14)$ \\
$3.33 / 2.90$ \\
$3.36 / 3.13$ \\
$3.54 / 2.43 / 3.00$ \\
$3.08 / 3.58 / 2.50$ \\
\end{tabular} \\
\hline
\end{tabular}




\begin{tabular}{|c|c|c|c|c|c|}
\hline & Frec. de uso & imaymacioni & Comprensión & Depresión & Ansiedad \\
\hline 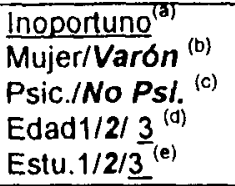 & \begin{tabular}{|l|}
$5.54(3.09)$ \\
$5.58 / 5.37$ \\
$6.01 / 5.15\left(^{*}\right)$ \\
$5.83 / 4.45 / 6.50\left(^{*}\right)$ \\
$5.31 / 5.63 / 5.31$ \\
\end{tabular} & $\begin{array}{l}6.38(3.39) \\
6.31 / 6.68 \\
6.24 / 6.58 \\
6.34 / 6.57 / 7.25 \\
6.15 / 6.43 / 6.28 \\
\end{array}$ & $\begin{array}{l}8.48(2.39) \\
8.48 / 8.45 \\
8.77 / 8.21 \\
8.41 / 8.86 / 8.13 \\
7.92 / 8.33 / 8.88 \\
\end{array}$ & $\begin{array}{l}2.71(2.91) \\
2.61 / 3.02 \\
3.18 / 2.33(") \\
2.81 / 2.33 / 2.25 \\
3.00 / 2.64 / 2.79\end{array}$ & $\begin{array}{l}3.66(3.43) \\
3.61 / 3.84 \\
3.94 / 3.45 \\
3.89 / 2.96 / 4.63 \\
4.23 / 3.99 / 2.90\end{array}$ \\
\hline 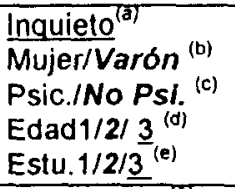 & $\begin{array}{l}6.70(2.85) \\
6.78 / 6.39 \\
7.27 / 6.20\left(^{*}\right) \\
6.62 / 6.86 / 7.57 \\
6.42 / 6.52 / 7.19 \\
\end{array}$ & \begin{tabular}{|l|}
$7.71(2.85)$ \\
$7.73 / 7.75$ \\
$8.23 / 7.32$ \\
$7.71 / 7.53 / 9.14$ \\
$7.25 / 7.61 / 8.00\left(^{*}\right)$ \\
\end{tabular} & $\begin{array}{l}8.82(2.08) \\
8.89 / 8.59 \\
9.20 / 8.48\left(^{\star}\right) \\
8.71 / 9.10 / 9.57 \\
8.67 / 8.58 / 9.34 \\
\end{array}$ & $\begin{array}{l}3.27(3.06) \\
3.32 / 3.00 \\
2.99 / 3.46 \\
3.04 / 3.65 / 5.57 \\
5.17 / 3.09 / 3.36 \\
\end{array}$ & \begin{tabular}{|l|}
$8.38(2.43)$ \\
$8.43 / 8.22$ \\
$9.07 / 7.77\left(^{\star}\right)$ \\
$8.65 / 7.51 / 8.57\left(^{\star}\right)$ \\
$7.08 / 8.47 / 8.48\left(^{\star}\right)$
\end{tabular} \\
\hline 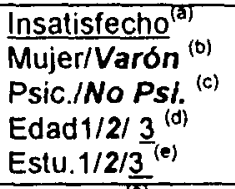 & $\begin{array}{l}5.58(2.95) \\
5.50 / 5.78 \\
6.02 / 5.19\left(^{\star \prime}\right) \\
5.68 / 5.00 / 6.88 \\
6.15 / 5.50 / 5.56 \\
\end{array}$ & \begin{tabular}{|l|}
$6.67(3.09)$ \\
$6.64 / 6.86$ \\
$6.85 / 6.58$ \\
$6.61 / 7.20 / 5.38$ \\
$5.85 / 6.63 / 6.93$ \\
\end{tabular} & \begin{tabular}{|l}
$8.72(2.07)$ \\
$8.69 / 8.78$ \\
$9.06 / 8.41\left(^{\star}\right)$ \\
$8.67 / 8.78 / 9.13$ \\
$8.23 / 8.55 / 9.15$ \\
\end{tabular} & $\begin{array}{l}7.04(3.13) \\
7.12 / 6.76 \\
7.41 / 6.76 \\
7.19 / 6.76 / 6.25 \\
5.92 / 7.04 / 7.36 \\
\end{array}$ & $\begin{array}{l}6.57(3.16) \\
6.63 / 6.49 \\
6.27 / 6.91 \\
6.61 / 6.76 / 5.75 \\
7.15 / 6.85 / 5.78 \\
\end{array}$ \\
\hline $\begin{array}{l}\text { Insequro }^{|0|} \\
\text { Mujer/Varón }^{(\mathrm{b})} \\
\text { Psic./No Ps|. } \\
{\text { Edad } 1 / 2 / 3^{(\mathrm{d})}}^{(\mathrm{d})} \\
\text { Estu.1/2/3 }^{(\mathrm{e})}\end{array}$ & $\begin{array}{l}6.56(2.98) \\
6.74 / 5.92 \\
7.05 / 6.13\left(^{\star}\right) \\
6.79 / 5.82 / 6.71 \\
5.62 / 6.44 / 6.98 \\
\end{array}$ & $\begin{array}{l}6.94(3.07) \\
6.94 / 6.96 \\
7.05 / 6.85 \\
7.07 / 7.02 / 5.00 \\
6.92 / 6.89 / 6.98\end{array}$ & $\begin{array}{l}8.90(2.07) \\
8.99 / 8.61 \\
9.20 / 8.64\left(^{*}\right) \\
8.95 / 8.61 / 9.63 \\
8.31 / 8.88 / 9.02\end{array}$ & $\begin{array}{l}6.90(3.36) \\
6.87 / 6.96 \\
7.34 / 6.52 \\
6.89 / 6.90 / 7.00 \\
5.00 / 6.99 / 7.23 \\
\end{array}$ & $\begin{array}{l}7.42(3.01) \\
7.46 / 7.27 \\
7.73 / 7.19 \\
7.62 / 7.11 / 5.38 \\
7.46 / 7.69 / 6.77\end{array}$ \\
\hline $\begin{array}{l}\text { Insomnio }^{(2)} \\
\text { Mujer/Varón } \\
\text { Psic./No Ps/. }^{(\mathrm{c})} \\
\text { Edad } 1 / 2 / \underline{3}^{(\mathrm{d})} \\
\text { Estu.1/2/3 }^{(\mathrm{e})}\end{array}$ & \begin{tabular}{|l|}
$5.68(3.17)$ \\
$6.00 / 4.72\left(^{*}\right)$ \\
$6.70 / 4.83\left({ }^{*}\right)$ \\
$5.86 / 5.16 / 5.50$ \\
$3.23 / 5.64 / 6.32\left({ }^{*}\right)$ \\
\end{tabular} & $\begin{array}{l}7.82(2.86) \\
7.88 / 7.58 \\
8.32 / 7.35\left(^{\star}\right) \\
7.87 / 7.90 / 5.88 \\
6.00 / 7.82 / 8.12\left(^{*}\right) \\
\end{array}$ & $\begin{array}{l}9.03(2.12) \\
9.07 / 8.88 \\
9.47 / 8.65\left(^{\star}\right) \\
9.07 / 8.78 / 9.63 \\
7.69 / 9.04 / 9.28\left(^{\star}\right) \\
\end{array}$ & $\begin{array}{l}6.77(3.68) \\
7.18 / 5.48\left({ }^{*}\right) \\
7.44 / 6.20\left({ }^{*}\right) \\
6.81 / 6.73 / 6.13 \\
5.85 / 6.41 / 7.93\left({ }^{*}\right) \\
\end{array}$ & $\begin{array}{l}7.77(3.09) \\
7.81 / 7.22 \\
8.27 / 7.19\left(^{*}\right) \\
7.89 / 7.57 / 4.50\left(^{*}\right) \\
5.92 / 8.04 / 7.40\left({ }^{\star}\right) \\
\end{array}$ \\
\hline $\begin{array}{l}\text { Intranquilo } \\
\text { Mujer/Varón }^{(b)} \\
\text { Psic./No Psi. } \\
{\text { Edad } 1 / 2 / \underline{3}^{(d)}}^{\text {(d) }} \\
{\text { Estu. } 1 / 2 / 3^{(e)}}^{\text {(e) }}\end{array}$ & $\begin{array}{l}6.64(3.10) \\
6.99 / 5.53\left(^{*}\right) \\
6.89 / 6.46 \\
6.64 / 6.69 / 8.25 \\
6.92 / 6.45 / 7.17 \\
\end{array}$ & $\begin{array}{l}7.74(2.83) \\
7.70 / 7.88 \\
7.95 / 7.62 \\
7.79 / 7.69 / 8.38 \\
7.00 / 7.72 / 8.02 \\
\end{array}$ & $\begin{array}{l}9.13(1.67) \\
9.13 / 9.10 \\
9.34 / 8.93 \\
9.16 / 9.12 / 8.75 \\
8.54 / 9.10 / 9.29 \\
\end{array}$ & $\begin{array}{l}5.40(3.52) \\
5.63 / 4.65 \\
5.23 / 5.50 \\
5.21 / 5.86 / 6.00 \\
6.00 / 5.44 / 5.22 \\
\end{array}$ & $\begin{array}{l}8.96(2.09) \\
8.96 / 8.94 \\
9.41 / 8.57\left(^{*}\right) \\
9.09 / 8.55 / 8.99 \\
8.69 / 8.94 / 9.21 \\
\end{array}$ \\
\hline 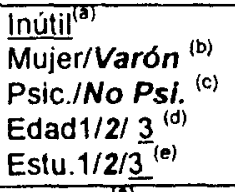 & \begin{tabular}{|l|}
$6.15(3.29)$ \\
$6.18 / 6.02$ \\
$6.78 / 5.61\left(^{\star}\right)$ \\
$6.36 / 5.39 / 7.00$ \\
$6.46 / 5.90 / 6.64$ \\
\end{tabular} & $\begin{array}{l}6.64(3.24) \\
6.52 / 7.10 \\
6.79 / 6.58 \\
6.54 / 7.04 / 7.25 \\
6.54 / 6.50 / 6.97 \\
\end{array}$ & \begin{tabular}{|l|}
$8.91(2.11)$ \\
$8.93 / 8.86$ \\
$9.26 / 8.61\left(^{*}\right)$ \\
$8.92 / 8.80 / 9.25$ \\
$8.15 / 8.82 / 9.24$ \\
\end{tabular} & \begin{tabular}{|l|}
$6.18(3.53)$ \\
$6.31 / 5.75$ \\
$6.97 / 5.49\left(^{\star}\right)$ \\
$6.24 / 5.98 / 6.38$ \\
$6.08 / 6.00 / 6.50$ \\
\end{tabular} & $\begin{array}{l}3.81(3.51) \\
3.94 / 3.47 \\
3.18 / 4.39\left(^{\star}\right) \\
3.87 / 3.69 / 4.75 \\
4.92 / 4.21 / 2.76 \\
\end{array}$ \\
\hline 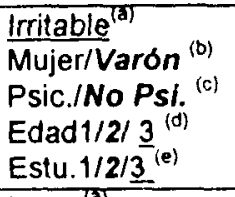 & $\begin{array}{l}5.36(3.02) \\
5.64 / 4.43\left(^{*}\right) \\
6.13 / 4.69\left(^{\star}\right) \\
5.37 / 5.53 / 3.75 \\
5.23 / 5.03 / 6.07 \\
\end{array}$ & $\begin{array}{l}7.22(3.92) \\
7.12 / 7.55 \\
7.59 / 6.94 \\
7.19 / 7.39 / 7.50 \\
6.38 / 7.07 / 7.74 \\
\end{array}$ & \begin{tabular}{|l|}
$8.82(2.13)$ \\
$8.93 / 8.49$ \\
$9.33 / 8.39\left(^{\star}\right)$ \\
$8.72 / 9.24 / 8.88$ \\
$8.23 / 8.64 / 9.34$ \\
\end{tabular} & \begin{tabular}{|l|}
$5.40(3.71)$ \\
$5.51 / 4.98$ \\
$5.31 / 5.42$ \\
$5.24 / 5.92 / 5.50$ \\
$5.85 / 5.45 / 5.28$ \\
\end{tabular} & $\begin{array}{l}8.09(2.70) \\
8.17 / 7.86 \\
8.56 / 7.69\left(^{*}\right) \\
8.20 / 7.88 / 8.63 \\
7.69 / 8.17 / 8.16 \\
\end{array}$ \\
\hline 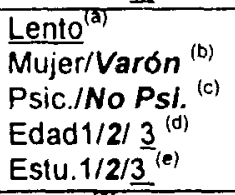 & $\begin{array}{l}7.17(2.80) \\
7.33 / 6.63 \\
7.75 / 6.67\left(^{*}\right) \\
7.41 / 6.29 / 8.13\left({ }^{*}\right) \\
7.00 / 7.09 / 7.36 \\
\end{array}$ & $\begin{array}{l}7.42(3.07)^{\prime} i \\
7.36 / 7.61 \\
7.86 / 7.07 \\
7.46 / 7.35 / 7.75 \\
6.62 / 7.34 / 7.79\end{array}$ & $\begin{array}{l}8.98(2.07) \\
9.07 / 8.69 \\
9.40 / 8.61\left(^{*}\right) \\
8.99 / 8.78 / 9.50 \\
8.15 / 8.93 / 9.24 \\
\end{array}$ & \begin{tabular}{|l|}
$5.05(3.91)$ \\
$5.16 / 4.71$ \\
$6.75 / 3.58\left(^{*}\right)$ \\
$5.17 / 4.65 / \underline{3.13}$ \\
$3.85 / 4.64 / 6.26\left({ }^{*}\right)$ \\
\end{tabular} & \begin{tabular}{|l|}
$1.71(2.55)$ \\
$1.60 / 2.08$ \\
$1.67 / 1.75$ \\
$1.76 / 1.63 / 0.88$ \\
$2.08 / 1.75 / 1.59$ \\
\end{tabular} \\
\hline $\begin{array}{l}\text { Llanto }^{(0)} \\
\text { Mujer/Varón } \\
\text { Psic./No Psi. } \\
\text { (c) } \\
\text { Edad1/2/ }{ }^{\text {(d) }} \\
{\text { Estu. } 1 / 2 / 3^{(e)}}\end{array}$ & $\begin{array}{l}5.92(3.43) \\
6.07 / 5.38 \\
6.63 / 5.29\left(^{*}\right) \\
6.11 / 5.56 / 5.00 \\
4.38 / 5.85 / 6.47 \\
\end{array}$ & $\begin{array}{l}8.47(2.57) \\
8.64 / 7.88 \\
8.92 / 8.06\left({ }^{*}\right) \\
8.55 / 8.44 / 7.75 \\
7.77 / 8.48 / 8.57 \\
\end{array}$ & \begin{tabular}{|l|}
$9.14(1.76)$ \\
$9.23 / 8.86$ \\
$9.40 / 8.92\left(^{\star}\right)$ \\
$9.07 / 9.33 / 9.25$ \\
$8.92 / 9.06 / 9.37$ \\
\end{tabular} & \begin{tabular}{|l}
$8.24(2.72)$ \\
$8.36 / 7.84$ \\
$9.11 / 7.50\left({ }^{\star}\right)$ \\
$8.39 / 8.14 / 7.25$ \\
$8.31 / 8.00 / 8.72$ \\
\end{tabular} & $\begin{array}{l}5.87(3.38) \\
5.91 / 5.84 \\
5.91 / 5.88 \\
6.19 / 5.53 / 3.38\left(^{*}\right) \\
4.38 / 6.49 / 4.79\left({ }^{*}\right) \\
\end{array}$ \\
\hline 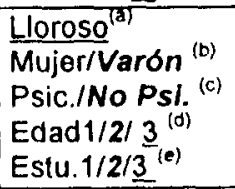 & $\begin{array}{l}5.75(3.29) \\
6.01 / 4.90\left(^{\star}\right) \\
6.16 / 5.39 \\
5.79 / 5.63 / 6.88 \\
6.46 / 5.41 / 6.49 \\
\end{array}$ & $\begin{array}{l}7.98(2.88) \\
8.11 / 7.53 \\
8.38 / 7.62\left(^{*}\right) \\
8.15 / 7.57 / 8.00 \\
6.92 / 7.97 / 8.22\end{array}$ & \begin{tabular}{|l}
$8.95(2.08)$ \\
$9.04 / 8.67$ \\
$9.07 / 8.84$ \\
$8.93 / 8.90 / 9.50$ \\
$9.23 / 8.93 / 8.93$ \\
\end{tabular} & $\begin{array}{l}8.31(2.57) \\
8.47 / 7.80 \\
8.75 / 7.93\left(^{\star}\right) \\
8.44 / 7.96 / 7.63 \\
7.85 / 8.18 / 8.67 \\
\end{array}$ & $\begin{array}{l}4.23(3.49) \\
4.23 / 4.32 \\
3.56 / 4.83\left(^{\star}\right) \\
4.06 / 5.06 / 3.68 \\
4.62 / 4.47 / 3.69 \\
\end{array}$ \\
\hline
\end{tabular}




\begin{tabular}{|c|c|c|c|c|c|}
\hline & Frec de uso & Imaginación & Comprensión & Depresión & Ansiedad \\
\hline Mareado $^{(\text {(2) }}$ & $6.46(3.14)$ & $7.50(3.06)$ & $8.79(2.19)$ & $3.85(3.33)$ & $4.21(3.41)$ \\
\hline Mujer/Varón ${ }^{\text {(b) }}$ & $6.65 / 5.80$ & $7.57 / 7.24$ & $8.86 / 8.57$ & $3.87 / 3.84$ & $4.47 / 3.51$ \\
\hline Psic./No Ps/. ${ }^{\left({ }^{c}\right)}$ & $7.28 / 5.73\left(^{*}\right)$ & $.94 / 7.09\left({ }^{*}\right)$ & $9.08 / 8.53$ & $4.40 / 3.42\left({ }^{\circ}\right)$ & $4.69 / 3.81$ \\
\hline $\operatorname{Edad} 1 / 2 / \underline{3}^{(d)}$ & $6.73 / 5.55 / 7.63\left(^{*}\right)$ & $7.57 / 7.45 / 7.43$ & $8.79 / 8.88 / \underline{9.13}$ & $4.02 / 3.51 / 3.75$ & $4.32 / 3.73 / 4.88$ \\
\hline $12 / \underline{3}^{(\mathrm{e})}$ & $.92 / 6.27 / \overline{6.80}$ & $7.08 / 7.38 / \overline{7.74}$ & $8.54 / 8.68 / 9.03$ & $3.85 / 3.78 / 4.14$ & $5.46 / 3.81 / 4.91\left(^{*}\right)$ \\
\hline Mareo $^{(\text {(a) }}$ & .30) & $7.59(3.01)$ & $8.79(2.15)$ & $3.79(3.40)$ & $4.95(3.56)$ \\
\hline Mujer/Varónn ${ }^{(b)}$ & 33 & $62 / 7.58$ & $8.87 / 8.53$ & $3.80 / 3.84$ & $5.07 / 4.69$ \\
\hline INo Psi. ${ }^{(c)}$ & $7.20 / 5.67\left(^{*}\right)$ & $7.98 / 7.29$ & $9.21 / 8.42\left(^{*}\right)$ & $4.47 / 3.27\left(^{*}\right)$ & $5.77 / 4.27\left({ }^{*}\right)$ \\
\hline Edad1/2/ $\underline{3}^{(d)}$ & $\left.6.69 / 5.13 / 8.633^{*}\right)$ & $7.64 / 7.77 / 6.88$ & $8.80 / 8.98 / 8.50$ & $3.95 / 3.67 / 2.88$ & $5.07 / 4.82 / 5.00$ \\
\hline Estu. $1 / 2 / \underline{3}^{(e)}$ & $7.77 / 6.28 / 6.29$ & $6.54 / 7.53 / 7.90$ & $8.00 / 8.71 / 9.12$ & $3.69 / 3.76 / 4.03$ & $5.46 / 4.46 / 6.10$ \\
\hline Miedo ${ }^{(\text {a) }}$ & $7.33(2.88$ & $8.11(2.69)$ & $9.11(1.80)$ & $6.02(3.55)$ & $8.87(2.05)$ \\
\hline Mujer/Varón ${ }^{\text {(b) }}$ & 7.5 & 8.13 & 9.14 & $6.27 / 5.27$ & $8.90 / 8.76$ \\
\hline |No Ps!. ${ }^{\text {(c) }}$ & $7.76 / 6.96\left(^{*}\right)$ & 8.28 & $9.24 / 9.00$ & 5.54 & $9.16 / 8.61$ \\
\hline$/ 2 / \underline{3}^{(d)}$ & 7.5 & $19 / 7.75$ & $41 / 9.13$ & $.31 / \underline{5.25}$ & $8.97 / 8.82 / 7.00$ \\
\hline Estu. 1/2/3 ${ }^{(e)}$ & $7.69 / 7.18 / 7.61$ & $15 / 8.18$ & $.05 / 9.29$ & $.84 / 6.29$ & $7.23 / 8.83 / 9.40$ \\
\hline Miedoso $^{(\text {II) }}$ & .11) & 7.64 & 8.83 & 5.5 & 7.7 \\
\hline Mujer/Varón ${ }^{(b)}$ & & 7.7 & 8.84 & & 7.9 \\
\hline (No Ps!. ${ }^{(c)}$ & $7.03 /$ & $7.71 / 7.58$ & $9.26 / 8.46\left(^{*}\right)$ & $5.22 / 5.79$ & $8.27 / 7.20\left(^{*}\right)$ \\
\hline $12 / 3^{10}$ & $6.87 / 5.94 / 6.71$ & $.73 / \underline{8.38}$ & $8.93 / 8.63 / 9.13$ & $.57 / 5.50$ & $7.91 / 7.37 / 5.63\left({ }^{*}\right)$ \\
\hline Estu. 1/2/3 ${ }^{(e)}$ & $5.92 /$ & 7.72 & $77 / 9.19\left(^{*}\right)$ & 15.36 & $\left./ 7.980^{*}\right)$ \\
\hline Molestia $^{(\mathfrak{a})}$ & 3 & 6.36 & $8.76(1.97)$ & 5.5 & 5.8 \\
\hline$\overline{\text { Mujer/Varón }}{ }^{\text {(b) }}$ & & & 8.82 & & \\
\hline No Psi. ${ }^{(c)}$ & $6.82 / 6$ & 6.47 & 8.86 & $6.19 / 4.94\left(^{*}\right)$ & $6.32 / 5.37\left(^{*}\right)$ \\
\hline & 6.4 & 6.4 & 18.63 & 16.38 & $23 / 4.75$ \\
\hline Es & & 6.7 & $3 / 9.07$ & $7 / 6.55\left(^{*}\right)$ & 6.33 \\
\hline Molesto & 6.17 & $\longdiv { 6 . 4 9 }$ & 8.5 & 40) & $5.86(3.17)$ \\
\hline Mujer/Varónn ${ }^{\text {(b) }}$ & $6.26 /$ & & & & 6.0 \\
\hline No Psi. (c) & 6.64 & 6.5 & 8.64 & 4.7 & 6.25 \\
\hline & 2 & .88 & & $4 / \underline{3.25}$ & $24 / \underline{\underline{3.63}}\left(^{*}\right)$ \\
\hline $12 / 3^{(e)}$ & $53(*)$ & 6.5 & .79 & 14.99 & $8 / 5.78$ \\
\hline Muerte $^{(\delta)}$ & $6.25(3.49)$ & 8.31 & $8.88(2.43)$ & $7.72(3.18)$ & 6.8 \\
\hline Mujer/Varó & 36 & 8.32 & 8.99 & 778 & 7.0 \\
\hline No Ps! & $.74(*)$ & $76\left(^{*}\right)$ & 9.33 & 8.2 & 6.8 \\
\hline $1 / 2 / \underline{3}^{(0)}$ & $6.75 / 4.73 / 5.86\left(^{*}\right)$ & $59 / \underline{8.13}$ & $2 / 8.00$ & $1 / 7.13$ & $.08 / \underline{4.88}\left({ }^{*}\right)$ \\
\hline $1 / 2 / 3^{(e)}$ & 5.5 & 7.3 & .29 & .93 & 6.40 \\
\hline Mutilado $^{(a)}$ & 3.31 & $\overline{7.78}$ & 27) & 6.5 & 5.05 \\
\hline Mujer/Varónn ${ }^{\text {(b) }}$ & & & 8.79 & 6.7 & 5.0 \\
\hline No Psi. ${ }^{(c)}$ & $3.57 / 3.10$ & $8.31 / 7.33\left(^{*}\right)$ & $9.09 / 8$ & $6.29 / 6.79$ & $4.44 / 5.64\left(^{*}\right)$ \\
\hline $12 / 3^{4}$ & $3.18 /$ & $7.68 / 7.96 / 8.75$ & 8.62 & 13 & $5.31 / 4.46 / 5$ \\
\hline $2 / \underline{3}^{6}$ & & & 8.3 & .32 & 3.7 \\
\hline Nervioso $^{(d)}$ & $7.99(2.56)$ & $8.28(2.33)$ & $9.00(1.84)$ & $4.24(3.54)$ & $8.96(2.00)$ \\
\hline Mujer/Varónn ${ }^{\text {(b) }}$ & $8.23 / 7.22\left({ }^{*}\right)$ & $8.35 / 8.08$ & $9.11 / 8.63$ & $4.37 / 3.80$ & $8.95 / 8.98$ \\
\hline Psic./No Psi. & $8.29 / 7.75$ & $8.45 / 8.13$ & $9.17 / 8.83$ & $4.00 / 4.42$ & $9.23 / 8.72$ \\
\hline Edad1/2/ $\underline{3}^{10}$ & 8.13 & 8.35 & 9.03 & $1.80 / 4.13$ & $9.10 / 8.16 / 9.25\left(^{*}\right)$ \\
\hline & 7.62 & 41 & $96 / \overline{9.09}$ & $4.54 / 4.34 / 3.93$ & $\left.7.92 / 8.29 / 8.410^{\circ}\right)$ \\
\hline Odioso $^{(\mathrm{a})}$ & $4.63(3.22)$ & $6.00(3.46)$ & $8.44(2.45)$ & $3.44(3.41)$ & $4.01(3.43)$ \\
\hline Mujer/Var & $4.77 / 4.16$ & $5.99 / 6.16$ & $8.43 / 8.50$ & $3.38 / 3.70$ & $3.98 / 4.16$ \\
\hline Psic./No Psi. & $5.10 / 4.22\left({ }^{*}\right)$ & $6.29 / 5.84$ & $8.82 / 8.11\left(^{*}\right)$ & $3.45 / 3.47$ & $4.00 / 4.06$ \\
\hline Edad $1 / 2 / \underline{3}^{(d)}$ & $4.78 / 3.90 / 6.38$ & $5.96 / 6.18$ & $8.40 / 8.53 / 8.50$ & $3.74 / 2.65 / 2.63$ & $4.18 / 3.29 / 6.38\left(^{*}\right)$ \\
\hline & $4.08 / 4.61 / 4.69$ & $6.15 / 6.00 / 5.95$ & $6.38 / 8.41 / 8.88\left({ }^{*}\right)$ & $2.31 / 3.61 / 3.28$ & $5.00 / 4.30 / 3.26$ \\
\hline Ofendido $^{(2)}$ & $5.86(2.92)$ & $6.39(3.22)$ & $8.71(2.09)$ & $4.72(3.49)$ & $4.53(3.43)$ \\
\hline Mujer/Varo & $5.92 / 5.63$ & $6.08 / 7.41\left(^{*}\right)$ & $8.67 / 8.86$ & $4.68 / 4.94$ & $4.77 / 3.80$ \\
\hline INO PSI. & $6.13 / 5.63$ & $6.38 / 6.47$ & $9.00 / 8.46$ & $5.16 / 4.38$ & $4.66 / 4.46$ \\
\hline Edad $1 / 2 / 3^{(1)}$ & $6.02 / 5.2$ & $6.46 / 6.33$ & $8.69 / 8.82 / 9.13$ & $5.07 / 3.85 / 4.00$ & $4.74 / 4.16 / 3.88$ \\
\hline Estu.1/2 & $5.67 / 5.83 / 5.92$ & $5.58 / 6.35 / 6.60$ & 7.15/8.74/8.95(*) & $3.15 / 4.85 / 4.78$ & $4.69 / 4.80 / 3.81$ \\
\hline
\end{tabular}




\begin{tabular}{|c|c|c|c|c|c|}
\hline & Frec. de uso & Imaginación & Comprensión & Depresión & Ansiedad \\
\hline Palp & $4.43(3.24)$ & $6.91(3.37)$ & $8.64(2.34)$ & $3.57(3.24)$ & $9.01(1.95)$ \\
\hline Mujer/Varónn ${ }^{(b)}$ & $4.63 / 3.80$ & $6.87 / 7.04$ & $8.73 / 8.35$ & $3.60 / 3.55$ & $9.14 / 8.67$ \\
\hline Psic./No Psl. ${ }^{(0)}$ & $5.27 / 3.72\left(^{*}\right)$ & $7.71 / 6.20\left(^{*}\right)$ & $9.14 / 8.20\left(^{*}\right)$ & $3.20 / 3.90$ & $9.65 / 8.49\left(^{*}\right)$ \\
\hline Edad $1 / 2 / \underline{3}^{(0)}$ & $4.30 / 4.67 / 5.88$ & $7.09 / 6.76 / 5.63$ & $8.67 / 8.63 / 9.00$ & $3.31 / 4.38 / 3.63$ & $9.14 / 8.69 / 8.50$ \\
\hline Estu.1/2/3 ${ }^{(e)}$ & $3.92 / 4.20 / 5.08$ & $4.54 / 6.80 / 7.59\left({ }^{*}\right)$ & $\left.6.54 / 8.61 / 9.14{ }^{\circ}\right)$ & $3.38 / 3.63 / 3.56$ & $7.46 / 9.04 / 9.33\left({ }^{*}\right)$ \\
\hline Pánico ${ }^{(2)}$ & $5.24(3.18)$ & $7.32(2.93)$ & $8.68(2.25)$ & $4.77(3.66)$ & $8.82(2.30)$ \\
\hline Mujer/Varónn ${ }^{\text {(b) }}$ & $5.33 / 4.92$ & $7.30 / 7.49$ & $8.61 / 8.88$ & $4.81 / 4.67$ & $8.92 / 8.49$ \\
\hline Psic./No Psl. ${ }^{(c)}$ & $6.09 / 4.50\left(^{*}\right)$ & $7.52 / 7.18$ & $8.85 / 8.52$ & $3.99 / 5.45\left(^{*}\right)$ & $9.14 / 8.54$ \\
\hline Edad1/2/ ${ }^{(d)}$ & $5.44 / 4.33 / 6.75\left(^{*}\right)$ & $7.23 / 7.53 / 8.50$ & $8.63 / 8.86 / 8.38$ & $4.91 / 4.41 / 5.00$ & $9.02 / 8.45 / 7.13\left(^{*}\right)$ \\
\hline Estu.1/2/3 ${ }^{(e)}$ & $4.92 / 5.18 / 5.36$ & $6.45 / 7.23 / 7.59$ & $8.23 / 8.66 / 8.78$ & $5.23 / 5.07 / 4.07$ & $6.92 / 8.96 / 8.90\left({ }^{*}\right)$ \\
\hline Paralizado & $4.40(3.27)$ & $7.15(2.98)$ & $8.55(2.28)$ & $4.99(3.78)$ & $4.89(3.86)$ \\
\hline Mujer/Varón ${ }^{(b)}$ & $4.48 / 4.10$ & $7.11 / 7.24$ & $8.67 / 8.16$ & $5.03 / 4.96$ & $5.14 / 4.16$ \\
\hline Psic./No Psi. ${ }^{(c)}$ & $5.23 / 3.65\left(\left(^{*}\right)\right.$ & $7.59 / 6.75$ & $8.83 / 8.28$ & $5.35 / 4.74$ & $5.57 / 4.32\left(^{*}\right)$ \\
\hline Edad1/2/ $\underline{3}^{(d)}$ & $4.42 / 3.79 / 7.25\left({ }^{*}\right)$ & $6.85 / 7.05 / 7.43$ & $8.41 / 9.00 / 8.13$ & $5.15 / 5.00 / 2.88$ & $4.98 / 4.69 / 5.38$ \\
\hline Estu. 1/2/3 ${ }^{(\theta)}$ & $4.85 / 4.19 / 4.66$ & $1 0 \longdiv { 7 . 5 0 }$ & $0 / 8.93$ & $5.38 / 4.85 / 5.29$ & $4.15 / 4.49 / 5.95\left({ }^{\circ}\right)$ \\
\hline Patético (d) & $3.55(3.23)$ & 5.27 & $7 . \overline{75}$ & $6.11(3.24)$ & $4.83(3.39)$ \\
\hline Mujer/Varón ${ }^{(6)}$ & 3.59 & $5.15 / 5.72$ & 7.7 & 6.1 & $4.87 / 4.76$ \\
\hline Psic./No Psl. ${ }^{\left({ }^{c}\right)}$ & $4.18 / 3.06\left({ }^{*}\right)$ & $5.34 / 5.27$ & $8.09 / 7.45$ & $6.53 / 5.81$ & $4.74 / 4.92$ \\
\hline Edad $1 / 2 / \underline{3}^{(d)}$ & $3.78 / 2.94 / 3.75$ & $5.28 / 5.53 / 4.83$ & $88 / 8.25$ & $6.21 / 6.02 / 6.50$ & $5.30 / 3.53 / 3.25\left(^{*}\right)$ \\
\hline Estu. $1 / 2 / 3^{(e)}$ & $3.31 / 3.41 / 4.02$ & $4.50 / 5.19 / 5.53$ & $6.69 / 7.68 / 8.05$ & $5.54 / 6.33 / 5.81$ & $3.08 / 5.18 / 4.38\left({ }^{\circ}\right)$ \\
\hline Peligro $^{(2)}$ & $6.84(3.07)$ & $8.17(2.61)$ & $8.99(2.06)$ & $3.12(3.25)$ & $7.00(3.44)$ \\
\hline Mujer/Varón ${ }^{(0)}$ & $7.00 / 6.26$ & $8.17 / 8.12$ & $9.07 / 8.68$ & $3.18 / 2.98$ & $7.17 / 6.46$ \\
\hline Psic./No Psi ${ }^{(G)}$ & $7.41 / 6.33\left(^{*}\right)$ & $8.40 /$ & $9.33 / 8.68\left({ }^{*}\right)$ & $3.37 / 2.92$ & $7.84 / 6.27\left(^{*}\right)$ \\
\hline Edad $1 / 2 / \underline{3}^{(d)}$ & $11 / 6.00$ & $8.19 / 8.12 / 7.63$ & $8.88 / 9.27 / 8.63$ & $2.97 / 3.69 / 3.38$ & $7.53 / 5.80 / 5.63\left(^{*}\right)$ \\
\hline Estu.1/2/3 $\underline{-}^{-(e)}$ & $9 9 \longdiv { 7 . 2 9 }$ & $6.77 / 8.25 / 8.19$ & 19.20 & $93 / 3.31$ & $.22 / 6.81$ \\
\hline Peor ${ }^{(3)}$ & $7.10(3.29)$ & $5.24(3.63)$ & $8.70(2.30)$ & $6.26(3.55)$ & $4.69(3.49)$ \\
\hline Mujer/Varónn ${ }^{\text {(b) }}$ & $7.23 / 6.66$ & $4.99 / 6.06$ & 8.80 & $6.43 / 5.76$ & $4.71 / 4.73$ \\
\hline Psic./No Psl. ${ }^{(0)}$ & $7.75 / 6.57\left(^{*}\right)$ & $4.60 / 5.84\left(^{*}\right)$ & $8.93 / 8.50$ & $7.11 / 5.52\left(^{*}\right)$ & $5.15 / 4.30$ \\
\hline Edad1/2/ $\underline{3}^{(0)}$ & $7.37 / 6.15 / 8.75\left(^{*}\right)$ & $5.21 / 5.20 / 7.63$ & $8.75 / 8.63 / 9.25$ & $6.32 / 6.24 / 5.38$ & $4.79 / 4.42 / 500$ \\
\hline Estu. 1/2/3 & $8.08 / 6.74 / 7.71$ & $6.15 / 5.08 / 5.38$ & 19.27 & $\left.9 \longdiv { 7 . 0 5 } { } ^ { * }\right)$ & $3.69 / 4.80 / 4.64$ \\
\hline Perplejo $^{(\text {f) }}$ & $3.61(2.97)$ & $5.66(3.34)$ & $7.74(2.66)$ & $2.89(2.96)$ & $3.56(2.98)$ \\
\hline Mujer/Varónn ${ }^{(0)}$ & $3.58 / 3.66$ & $5.72 / 5.52$ & $7.84 / 7.40$ & $91 / 2.88$ & $3.76 / 2.96$ \\
\hline Psic./No Psl. & $3.91 / 3.37$ & $5.67 / 5.71$ & $8.01 / 7$ & 2.47 & $3.88 / 3.33$ \\
\hline Edad $1 / 2 / \underline{3}^{(\alpha)}$ & $3.66 / 3.45 / 3.88$ & $5.59 / 5.92 / 6.25$ & $7.56 / 8.18 / 8.63$ & $3.16 / 2.22 / 2.13$ & $3.70 / 3.22 / 3.63$ \\
\hline Estu. $1 / 2 / 3^{(e)}$ & $3.23 / 3.66 / \overline{3.54}$ & $5.62 / 5.55 / 5.86$ & 7.38 & $2.31 / 2.88 / \overline{3.09}$ & $4.00 / 3.44 / 3.83$ \\
\hline Persequido & $4.69(3.23)$ & $7.96(2.78)$ & $8.88(2.21)$ & $4.20(3.46)$ & $8.36(2.72)$ \\
\hline Mujer/Varón ${ }^{\text {(b) }}$ & $4.84 / 4.14$ & $8.11 / 7.61$ & $8.95 / 8.63$ & 4.27 & $8.56 / 7.78$ \\
\hline Psic./No Psl. ${ }^{(c)}$ & $5.65 / 3.88\left(^{*}\right)$ & $8.42 / 7.62\left(^{*}\right)$ & $9.15 / 8.63$ & $3.82 / 4.58$ & $8.56 / 8.27$ \\
\hline Edad $1 / 2 / \underline{3}^{(d)}$ & $4.81 / 4.22 / 5.00$ & $8.07 / 7.71 / 8.13$ & $8.77 / 9.24 / 9.00$ & $4.24 / 4.25 / 5.38$ & $8.64 / 7.90 / 7.13$ \\
\hline Estu. 1/2/3 ${ }^{(\mathrm{e})}$ & $4.31 / 4.42 / 5.39$ & $6.38 / 7.99 / 8.19$ & 9.36 & $6 / 4.19$ & $\left.8.77 / 7.860^{\circ}\right)$ \\
\hline Pobre $^{(8)}$ & $7.07(2.84)$ & $8.20(2.71)$ & $9.04(2.11)$ & $4.39(3.68)$ & $3.35(3.44)$ \\
\hline Mujer/Varón ${ }^{(0)}$ & $7.27 / 6.39$ & $8.18 / 8.24$ & $9.03 / 9.04$ & $4.32 / 4.69$ & $3.33 / 3.39$ \\
\hline Psic./No Psi. (c) & $7.66 / 6.57\left(^{*}\right)$ & $8.51 / 7.90$ & $9.31 / 8.79$ & $4.17 / 4.64$ & $3.28 / 3.42$ \\
\hline Edad1/2/ $\underline{3}^{(d)}$ & $7.27 / 6.27 / 8.50\left({ }^{*}\right)$ & $8.21 / 8.12 / 8.38$ & $9.13 / 9.02 / 8.13$ & $4.78 / 3.27 / 4.88\left({ }^{*}\right)$ & $3.71 / 2.67 / 1.63$ \\
\hline Estu. $1 / 2 / \underline{3}^{-(e)}$ & $7.85 / 6.88 / 7.34$ & $7.69 / 8.18 / 8.28$ & .12 & $5.00 / 4.75 / 3.340^{*}$ & $4.00 / 3.68 / 2.50$ \\
\hline Preocupación ${ }^{(3)}$ & $7.52(2.46)$ & $7.33(2.98)$ & $9.12(1.76)$ & $7.11(3.27)$ & $8.35(2.30)$ \\
\hline Mujer/Varón ${ }^{(b)}$ & $7.72 / 6.86\left({ }^{*}\right)$ & $7.30 / 7.51$ & $9.21 / 8.80$ & $7.35 / 6.37$ & $8.44 / 8.06$ \\
\hline Psic./No Psl. ${ }^{\left({ }^{(c)}\right.}$ & $7.88 / 7.23\left(^{*}\right)$ & $7.26 / 7.47$ & $9.42 / 8.85\left(^{*}\right)$ & $7.48 / 6.77$ & $8.52 / 8.19$ \\
\hline $\operatorname{Edad} 1 / 2 / \underline{3}^{(d)}$ & $7.47 / 7.43 / 9.38$ & $7.28 / 7.82 / 7.63$ & $9.09 / 9.16 / 9.50$ & $7.08 / 7.02 / 7.50$ & $8.38 / 8.54 / 6.75$ \\
\hline Estu. $1 / 2 / \underline{3}^{(e)}$ & $8.69 / 7.33 / 7.69$ & $7 . 6 2 / 7 . 3 4 \longdiv { 7 . 2 2 }$ & $8.69 / 9.02 / 9.39$ & $5 . 9 2 / 6 . 9 8 \longdiv { 7 . 7 2 }$ & $7.54 / 8.34 / 8.47$ \\
\hline Preocupado $^{(2)}$ & $1.67(2.61)$ & $7.99(2.52)$ & $9.18(1.58)$ & $6.85(3.29)$ & $8.06(2.40)$ \\
\hline$\overline{\text { Mujer/Varón }}{ }^{\text {(b) }}$ & 7.89/6.96(*) & & $9.26 / 8.94$ & $6.87 / 6.76$ & $8.11 / 7.88$ \\
\hline Psic./No PsI. & $8.03 / 7.38$ & $8.21 / 7.82$ & $9.25 / 9.11$ & $7.23 / 6.50$ & $8.23 / 7.90$ \\
\hline Edad $1 / 2 / 3^{10}$ & $7.79 / 7.29 / 8.75$ & $8.01 / 8.10 / 8.50$ & $9.17 / 9.24 / 9.13$ & $6.80 / 6.78 / 7.25$ & $7.99 / 8.33 / 7.50$ \\
\hline Estu. 1/2/3 & $8.38 / 7.44 / 8.03$ & $7.85 / 7.90 / 8.23$ & $8.77 / 9.14 / 9.32$ & $6.15 / 6.63 / 7.50$ & $7.85 / 8.04 / 8.17$ \\
\hline
\end{tabular}




\begin{tabular}{|c|c|c|c|c|c|}
\hline & Frec. de uso & Imaginación & Comprensión & Depresión & Ansiedad \\
\hline 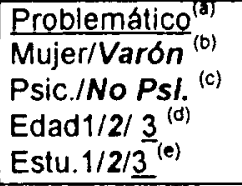 & \begin{tabular}{|l|}
$5.61(3.04)$ \\
$5.61 / 5.59$ \\
$6.09 / 5.20\left({ }^{*}\right)$ \\
$5.59 / 5.63 / 6.00$ \\
$5.62 / 5.34 / 6.20$ \\
\end{tabular} & \begin{tabular}{|l|}
$6.25(3.18)$ \\
$6.16 / 6.57$ \\
$6.27 / 6.29$ \\
$6.10 / 6.67 / 6.88$ \\
$6.33 / 6.14 / 6.43$ \\
\end{tabular} & \begin{tabular}{|l|}
$8.50(2.10)$ \\
$8.56 / 8.35$ \\
$8.91 / 8.16\left(^{*}\right)$ \\
$8.51 / 8.59 / 7.88$ \\
$6.54 / 8.42 / 9.05\left({ }^{*}\right)$
\end{tabular} & \begin{tabular}{|l|}
$5.72(3.48)$ \\
$5.83 / 5.43$ \\
$5.88 / 5.62$ \\
$5.87 / 5.69 / 4.50$ \\
$4.23 / 5.94 / 5.74$ \\
\end{tabular} & \begin{tabular}{|l|}
$6.32(3.31)$ \\
$6.66 / 5.22\left({ }^{*}\right)$ \\
$6.91 / 5.83\left(^{*}\right)$ \\
$6.48 / 5.92 / 6.25$ \\
$6.08 / 6.53 / 6.07$ \\
\end{tabular} \\
\hline 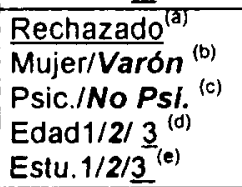 & $\begin{array}{l}5.36(3.05) \\
5.52 / 4.80 \\
5.84 / 4.93\left(^{*}\right) \\
5.49 / 4.52 / 7.13\left(^{*}\right) \\
6.31 / 5.28 / 5.32\end{array}$ & $\begin{array}{l}6.90(3.05) \\
6.89 / 6.94 \\
7.09 / 6.75 \\
6.83 / 7.38 / 5.63 \\
5.77 / 6.88 / 7.21 \\
\end{array}$ & \begin{tabular}{|l|}
$8.80(1.98)$ \\
$8.81 / 8.75$ \\
$8.99 / 8.62$ \\
$8.76 / 9.02 / 8.75$ \\
$8.15 / 8.76 / 8.97$ \\
\end{tabular} & \begin{tabular}{|l|}
$8.10(2.57)$ \\
$8.03 / 8.35$ \\
$8.41 / 7.86$ \\
$8.26 / 8.02 / 6.38$ \\
$7.00 / 8.32 / 7.88$ \\
\end{tabular} & \begin{tabular}{|l|}
$5.70(3.55)$ \\
$5.84 / 5.37$ \\
$5.75 / 5.73$ \\
$5.72 / 6.06 / 4.75$ \\
$4.15 / 6.08 / 5.22$
\end{tabular} \\
\hline 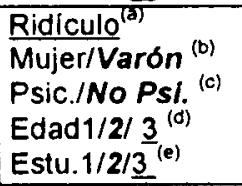 & $\begin{array}{l}5.11(3.20) \\
5.12 / 5.02 \\
5.52 / 4.73 \\
5.33 / 4.10 / 6.88\left({ }^{*}\right) \\
5.46 / 4.99 / 5.22 \\
\end{array}$ & $\begin{array}{l}7.36(2.88) \\
7.42 / 7.22 \\
7.66 / 7.12 \\
7.41 / 7.12 / 8.13 \\
6.77 / 7.35 / 7.45 \\
\end{array}$ & \begin{tabular}{|l|}
$8.61(2.29)$ \\
$8.62 / 8.56$ \\
$8.98 / 8.27\left(^{*}\right)$ \\
$8.65 / 8.24 / 9.63$ \\
$8.00 / 8.49 / 8.93$ \\
\end{tabular} & $\begin{array}{l}6.43(3.48) \\
6.33 / 6.84 \\
6.00 / 6.86 \\
6.61 / 5.92 / 6.63 \\
6.69 / 6.67 / 5.78 \\
\end{array}$ & \begin{tabular}{|l|}
$5.83(3.80)$ \\
$5.90 / 5.57$ \\
$5.75 / 5.88$ \\
$6.06 / 5.18 / 4.88\left(^{*}\right)$ \\
$5.08 / 6.21 / 5.14$ \\
\end{tabular} \\
\hline 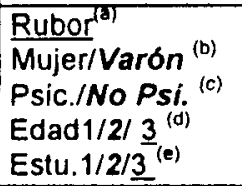 & \begin{tabular}{|l|}
$4.99(3.17)$ \\
$5.00 / 4.88$ \\
$5.56 / 4.49\left({ }^{*}\right)$ \\
$5.21 / 4.49 / 4.25$ \\
$4.15 / 4.82 / 5.53$ \\
\end{tabular} & \begin{tabular}{|l|}
$7.91(2.88)$ \\
$8.02 / 7.51$ \\
$8.49 / 7.39$ \\
$8.08 / 7.84 / 5.63$ \\
$5.77 / 7.88 / 8.36$ \\
\end{tabular} & $\begin{array}{l}44) \\
2 \\
3\left({ }^{*}\right) \\
3 / 8.25 \\
5 / 8.92\end{array}$ & \begin{tabular}{|l|}
$2.84(3.05)$ \\
$3.00 / 2.41$ \\
$2.94 / 2.78$ \\
$2.90 / 2.73 / 1.88$ \\
$1.77 / 3.11 / 2.50$ \\
\end{tabular} & \begin{tabular}{|l|}
$6.17(3.69)$ \\
$6.55 / 4.90\left({ }^{*}\right)$ \\
$7.09 / 5.34$ \\
$6.58 / 4.96 / 4.38\left({ }^{*}\right)$ \\
$4.92 / 6.41 / 5.78$ \\
\end{tabular} \\
\hline 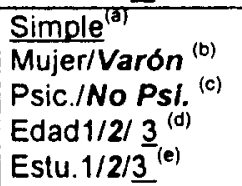 & $\begin{array}{l}6.04(3.28) \\
6.18 / 5.55 \\
6.46 / 5.66 \\
6.33 / 5.33 / 5.38 \\
5.54 / 5.88 / 6.52\end{array}$ & $\begin{array}{l}61) \\
8(*) \\
8 \\
2 / 5.13 \\
2 / 5.28\end{array}$ & $\begin{array}{l}25) \\
3 \\
1 \\
0 / 9.13 \\
6 / 9.17(*)\end{array}$ & \begin{tabular}{|l}
$1.84(2.59)$ \\
$1.91 / 1.65$ \\
$2.10 / 1.64$ \\
$1.87 / 1.55 / 3.25$ \\
$2.31 / 1.80 / 190$ \\
\end{tabular} & \begin{tabular}{|l}
$.56(2.24)$ \\
$1.50 / 1.78$ \\
$1.66 / 1.50$ \\
$1.52 / 1.67 / 1.25$ \\
$1.77 / 1.49 / 1.67$
\end{tabular} \\
\hline 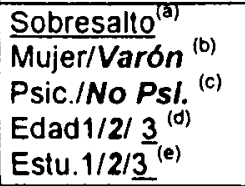 & \begin{tabular}{|l|}
$5.29(2.74)$ \\
$5.34 / 5.10$ \\
$5.48 / 5.11$ \\
$5.28 / 5.06 / 5.63$ \\
$6.00 / 5.24 / 5.22$
\end{tabular} & \begin{tabular}{|l}
$7.57(2.56)$ \\
$7.50 / 7.78$ \\
$7.67 / 7.46$ \\
$7.59 / 7.63 / 7.13$ \\
$7.08 / 7.53 / 7.67$
\end{tabular} & $\begin{array}{l}09) \\
49 \\
37 \\
33 / 8.50 \\
30 / 8.71 \\
\end{array}$ & \begin{tabular}{|l|}
$2.72(2.81)$ \\
$2.71 / 2.78$ \\
$2.65 / 2.80$ \\
$2.63 / 3.08 / 2.00$ \\
$3.38 / 2.77 / 2.53$ \\
\end{tabular} & \begin{tabular}{|l|}
$8.08(2.62)$ \\
$8.25 / 7.53$ \\
$8.63 / 7.61\left(^{*}\right)$ \\
$8.28 / 7.61 / 7.00$ \\
$7.31 / 8.19 / 7.98$ \\
\end{tabular} \\
\hline 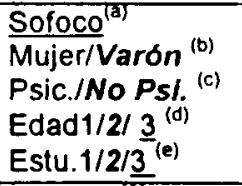 & \begin{tabular}{|l|}
$5.37(3.07)$ \\
$5.46 / 5.06$ \\
$5.92 / 4.92\left({ }^{*}\right)$ \\
$5.57 / 4.82 / 5.75$ \\
$5.23 / 5.20 / 5.81$ \\
\end{tabular} & \begin{tabular}{|l|}
$7.51(2.84)$ \\
$7.48 / 7.59$ \\
$8.18 / 6.93$ \\
$7.63 / 7.22 / 6.75$ \\
$4.54 / 7.53 / 8.05\left({ }^{*}\right)$ \\
\end{tabular} & $\begin{array}{l}36) \\
7\left({ }^{*}\right) \\
7 / 8.88 \\
3 / 8.76 \\
\end{array}$ & \begin{tabular}{|l|}
$2.93(2.95)$ \\
$2.87 / 3.20$ \\
$2.78 / 3.08$ \\
$2.92 / 3.15 / 1.63$ \\
$2.77 / 3.02 / 2.76$ \\
\end{tabular} & \begin{tabular}{|l|}
$7.19(3.15)$ \\
$7.31 / 6.78$ \\
$8.13 / 6.36\left({ }^{*}\right)$ \\
$7.64 / 5.90 / 6.00\left({ }^{*}\right)$ \\
$5.38 / 7: 20 / 7.59\left({ }^{*}\right)$
\end{tabular} \\
\hline $\begin{array}{l}\text { Solitario }^{(a)} \\
\text { Mujer/Varón }^{\left({ }^{(b)}\right.} \\
\text { Psic./No Psi. } \\
\text { Edad1/2/ } \underline{3}^{\text {(d) }} \\
{\text { Estu. } 1 / 2 / \underline{3}^{(\mathrm{e})}}\end{array}$ & \begin{tabular}{|l|}
$6.50(2.87)$ \\
$6.64 / 6.02$ \\
$6.94 / 6.11\left(^{*}\right)$ \\
$6.62 / 5.94 / 7.75$ \\
$6.15 / 6.38 / 6.85$ \\
\end{tabular} & \begin{tabular}{|l|}
$8.48(2.45)$ \\
$8.47 / 8.47$ \\
$8.69 / 8.28$ \\
$8.52 / 8.24 / 8.63$ \\
$7.23 / 8.58 / 8.47$ \\
\end{tabular} & \begin{tabular}{|l|}
$9.33(1.51)$ \\
$9.36 / 9.25$ \\
$9.36 / 9.30$ \\
$9.23 / 9.63 / 9.38$ \\
$9.23 / 9.30 / 9.41$ \\
\end{tabular} & \begin{tabular}{|l|}
$7.99(2.86)$ \\
$8.05 / 7.76$ \\
$8.47 / 7.57\left({ }^{*}\right)$ \\
$7.96 / 8.04 / 7.25$ \\
$7.08 / 8.08 / 7.91$ \\
\end{tabular} & \begin{tabular}{|l|}
$4.76(3.56)$ \\
$4.84 / 4.59$ \\
$4.22 / 5.27\left(^{*}\right)$ \\
$4.60 / 5.52 / 4.13$ \\
$5.08 / 4.91 / 4.35$ \\
\end{tabular} \\
\hline 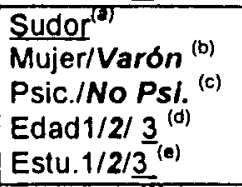 & $\begin{array}{l}(2.98) \\
6.84 \\
6.68\left(^{*}\right) \\
6.71 / 6.88 \\
7.01 / 7.66\end{array}$ & $\begin{array}{l}.72) \\
.26 \\
.58(*) \\
.92 / 8.63 \\
.31 / 8.58\end{array}$ & \begin{tabular}{|l|}
$8.94(2.79)$ \\
$8.91 / 9.02$ \\
$9.28 / 8.63\left({ }^{*}\right)$ \\
$9.01 / 8.78 / 9.38$ \\
$8.00 / 8.93 / 9.12$ \\
\end{tabular} & \begin{tabular}{|l|}
$2.79(3.06)$ \\
$2.75 / 2.96$ \\
$2.89 / 2.73$ \\
$2.61 / 3.51 / 2.13$ \\
$3.62 / 2.53 / 3.31$ \\
\end{tabular} & \begin{tabular}{|l|}
$7.21(3.50)$ \\
$7.26 / 7.02$ \\
$8.59 / 6.00\left({ }^{*}\right)$ \\
$7.56 / 6.47 / 5.13\left({ }^{*}\right)$ \\
$4.46 / 7.18 / 8.14\left(^{*}\right)$ \\
\end{tabular} \\
\hline $\begin{array}{l}\text { Sudoroso }^{\left({ }^{2}\right)} \\
\text { Mujer/Varón } \\
\text { Psic./No Psi }^{(\mathrm{b})} \\
\text { Edad11/2/3 }^{(\mathrm{d})} \\
\text { Estu.1/2/3 }^{(\mathrm{e})} \\
\end{array}$ & \begin{tabular}{|l|}
$5.53(3.23)$ \\
$5.53 / 5.44$ \\
$6.31 / 4.82\left({ }^{*}\right)$ \\
$5.48 / 5.37 / 8.00$ \\
$6.46 / 5.15 / 6.26$ \\
\end{tabular} & \begin{tabular}{|l|}
$7.92(2.35)$ \\
$7.98 / 7.68$ \\
$8.69 / 7.22\left({ }^{*}\right)$ \\
$7.88 / 8.06 / 8.00$ \\
$6.23 / 7.93 / 8.18$ \\
\end{tabular} & \begin{tabular}{|l|}
$8.78(2.20)$ \\
$8.76 / 8.82$ \\
$9.33 / 8.28\left(^{*}\right)$ \\
$8.84 / 8.65 / 8.50$ \\
$7.08 / 8.73 / 9.22\left({ }^{*}\right)$ \\
\end{tabular} & \begin{tabular}{|l|}
$2.97(2.98)$ \\
$3.03 / 2.82$ \\
$2.96 / 3.00$ \\
$2.86 / 3.33 / 2.50$ \\
$2.92 / 2.80 / 3.40$ \\
\end{tabular} & $\begin{array}{l}7.08(3.56) \\
7.35 / 6.16\left(^{*}\right) \\
8.66 / 5.70\left(^{*}\right) \\
7.24 / 6.65 / 5.75 \\
5.69 / 6.88 / 7.95\left({ }^{*}\right)\end{array}$ \\
\hline 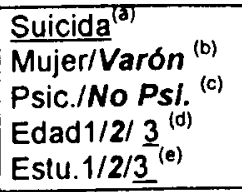 & \begin{tabular}{|l|}
$4.77(3.34)$ \\
$4.68 / 4.98$ \\
$5.46 / 4.14\left(^{*}\right)$ \\
$4.88 / 4.22 / 5.50$ \\
$3.62 / 4.73 / 5.03$ \\
\end{tabular} & $\begin{array}{l}7.83(2.99) \\
7.86 / 7.68 \\
8.49 / 7.22\left({ }^{*}\right) \\
7.78 / 7.84 / 7.50 \\
6.46 / 7.91 / 7.82 \\
\end{array}$ & \begin{tabular}{|l|}
$8.98(2.24)$ \\
$9.01 / 8.86$ \\
$947 / 8.55\left({ }^{*}\right)$ \\
$8.99 / 8.86 / 9.25$ \\
$6.85 / 9.03 / 9.29$ \\
\end{tabular} & \begin{tabular}{|l|}
$8.62(2.55)$ \\
$8.69 / 8.40$ \\
$8.52 / 8.71$ \\
$8.59 / 8.92 / 8.13$ \\
$8.31 / 8.62 / 8.67$ \\
\end{tabular} & \begin{tabular}{|l}
$6.63(3.55)$ \\
$6.82 / 6.43$ \\
$5.98 / 7.40\left(^{*}\right)$ \\
$6.56 / 7.27 / 7.25$ \\
$7.15 / 7.28 / 5.41$ \\
\end{tabular} \\
\hline
\end{tabular}




\begin{tabular}{|c|c|c|c|c|c|}
\hline & Frec. de uso & Imaginación & Comprensión & Depresión & Ansiedad \\
\hline $\begin{array}{l}\text { Suicidio }^{(8)} \\
\text { Mujer/Varón }^{\text {(b) }} \\
\text { Psic./No Psi. } \\
\text { Edad } 1 / 2 / 3^{\text {(d) }} \\
\text { Estu.1/2/3 }\end{array}$ & $\begin{array}{l}4.86(3.54) \\
4.89 / 4.67 \\
5.51 / 4.28\left(^{\star}\right) \\
4.97 / 4.35 / 5.38 \\
3.69 / 4.72 / 5.34\end{array}$ & $\begin{array}{l}8.36(2.69) \\
8.37 / 8.27 \\
8.69 / 8.04 \\
8.44 / 7.88 / 9.00 \\
8.54 / 8.42 / 8.09 \\
\end{array}$ & $\begin{array}{l}9.21(2.19) \\
9.24 / 9.12 \\
9.69 / 8.80\left(^{*}\right) \\
9.13 / 9.35 / 9.88 \\
8.69 / 9.11 / 9.54 \\
\end{array}$ & $\begin{array}{l}9.43(1.72) \\
9.45 / 9.35 \\
9.65 / 9.24 \\
9.41 / 9.59 / 9.38 \\
9.54 / 9.36 / 9.53 \\
\end{array}$ & $\begin{array}{l}7.44(3.36) \\
7.58 / 7.14 \\
6.75 / 8.12 \\
7.36 / 7.92 / 7.00 \\
7.62 / 7.92 / 6.30\left(^{\star}\right)\end{array}$ \\
\hline $\begin{array}{l}\text { Taquicardia }^{\text {(d) }} \\
\text { Mujer/Varón }^{\text {(b) }} \\
\text { Psic./No Psi. }^{\text {(c) }} \\
\text { Edad } 1 / 2 / \underline{3}^{\text {(d) }} \\
{\text { Estu. } 1 / 2 / \underline{3}^{(\mathrm{e})}}\end{array}$ & $\begin{array}{l}4.81(3.25) \\
3.86 / 3.58 \\
4.70 / 2.99\left(^{\star}\right) \\
3.75 / 3.67 / 5.25 \\
3.00 / 3.46 / 4.70\left(^{\star}\right)\end{array}$ & $\begin{array}{l}6.05(3.55) \\
6.06 / 6.13 \\
6.88 / 5.36\left(^{\star}\right) \\
5.73 / 7.00 / 6.50 \\
5.00 / 5.78 / 6.79 \\
\end{array}$ & \begin{tabular}{|l|}
$8.13(2.70)$ \\
$8.26 / 7.70$ \\
$8.64 / 7.68\left(^{*}\right)$ \\
$7.91 / 8.65 / 9.00$ \\
$6.69 / 7.93 / 8.83\left(^{*}\right)$ \\
\end{tabular} & $\begin{array}{l}3.51(3.31) \\
3.50 / 3.58 \\
2.83 / 4.13\left({ }^{*}\right) \\
3.28 / 4.35 / 4.13 \\
2.46 / 3.91 / 2.95 \\
\end{array}$ & \begin{tabular}{|l|}
$8.37(2.66)$ \\
$8.41 / 8.22$ \\
$9.13 / 7.71\left(^{*}\right)$ \\
$8.47 / 8.04 / 8.25$ \\
$6.38 / 8.39 / 8.74\left({ }^{*}\right)$ \\
\end{tabular} \\
\hline 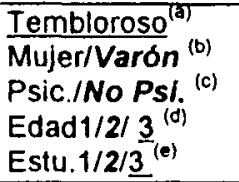 & $\begin{array}{l}4.51(3.30) \\
4.65 / 4.00 \\
5.39 / 3.74\left(^{*}\right) \\
4.51 / 4.40 / 5.88 \\
4.77 / 4.18 / 5.29\end{array}$ & \begin{tabular}{|l|}
$7.65(2.88)$ \\
$7.71 / 7.43$ \\
$8.40 / 7.00\left(^{*}\right)$ \\
$7.70 / 7.73 / 7.38$ \\
$7.00 / 7.36 / 8.45\left(^{*}\right)$ \\
\end{tabular} & $\begin{array}{l}8.83(2.09) \\
8.93 / 8.51 \\
9.18 / 8.53\left(^{*}\right) \\
8.78 / 9.20 / 8.50 \\
7.46 / 8.74 / 9.34\left(^{*}\right)\end{array}$ & $\begin{array}{l}5.51(3.41) \\
5.66 / 5.14 \\
5.36 / 5.67 \\
5.66 / 5.42 / 4.88 \\
6.77 / 5.60 / 5.14\end{array}$ & $\begin{array}{l}7.97(2.73) \\
8.16 / 7.31 \\
8.58 / 7.41\left(^{*}\right) \\
8.01 / 7.67 / 8.13 \\
7.69 / 7.99 / 8.07\end{array}$ \\
\hline 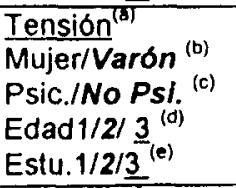 & $\begin{array}{l}6.25(2.91) \\
6.40 / 5.76 \\
6.96 / 5.66\left({ }^{*}\right) \\
6.20 / 6.31 / 6.75 \\
6.08 / 6.09 / 6.58 \\
\end{array}$ & $\begin{array}{l}7.05(2.95) \\
7.00 / 7.29 \\
7.20 / 6.95 \\
7.04 / 7.39 / 6.88 \\
6.31 / 6.89 / 7.47 \\
\end{array}$ & $\begin{array}{l}8.80(1.87) \\
8.89 / 8.47 \\
9.03 / 8.58 \\
8.69 / 9.14 / 8.63 \\
7.92 / 8.75 / 9.03 \\
\end{array}$ & $\begin{array}{l}4.95(3.56) \\
5.12 / 4.37 \\
4.56 / 5.23 \\
5.00 / 4.85 / 4.63 \\
5.54 / 4.91 / 4.98 \\
\end{array}$ & $\begin{array}{l}9.16(1.79) \\
9.38 / 8.43\left(^{*}\right) \\
9.48 / 8.87\left(^{*}\right) \\
9.30 / 8.82 / 8.25 \\
8.77 / 9.23 / 9.10 \\
\end{array}$ \\
\hline $\begin{array}{l}\text { Tenso }^{(0)} \\
\text { Mujer/Varón }^{(\mathrm{b})} \\
\text { Psic./No Psi. } \\
\text { Edad } 1 / 2 / 3^{(\mathrm{c})} \\
{\text { Estu. } 1 / 2 / 3^{(\mathrm{e})}}\end{array}$ & $\begin{array}{l}6.10(2.93) \\
6.23 / 5.67 \\
7.02 / 5.31\left(^{*}\right) \\
6.24 / 5.67 / 6.13 \\
5.31 / 5.90 / 6.67 \\
\end{array}$ & $\begin{array}{l}6.94(3.03) \\
6.85 / 7.35 \\
7.40 / 6.60\left(^{\star}\right) \\
6.96 / 6.98 / 7.75 \\
6.00 / 6.88 / 7.19 \\
\end{array}$ & $\begin{array}{l}8.56(2.20) \\
8.57 / 8.53 \\
9.01 / 8.16\left(^{\star}\right) \\
8.66 / 8.39 / 8.38 \\
6.85 / 8.57 / 8.85\left(^{\star}\right)\end{array}$ & $\begin{array}{l}4.13(3.40) \\
4.24 / 3.75 \\
3.79 / 4.38 \\
3.99 / 4.27 / 5.38 \\
4.46 / 4.23 / 3.91 \\
\end{array}$ & $\begin{array}{l}8.48(2.38) \\
8.55 / 8.22 \\
8.98 / 8.02\left(^{*}\right) \\
8.53 / 8.20 / 8.63 \\
7.08 / 8.41 / 8.93\left(^{*}\right) \\
\end{array}$ \\
\hline 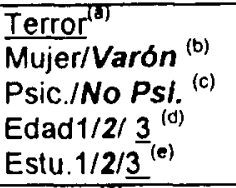 & $\begin{array}{l}5.55(3.28) \\
5.66 / 5.16 \\
6.21 / 4.97\left(^{\star}\right) \\
5.86 / 4.67 / 4.75 \\
4.54 / 5.48 / 5.85 \\
\end{array}$ & \begin{tabular}{|l|}
$8.05(2.62)$ \\
$7.98 / 8.39$ \\
$8.26 / 7.91$ \\
$8.11 / 8.06 / 8.00$ \\
$6.38 / 8.03 / 8.41\left(^{\circ}\right)$ \\
\end{tabular} & $\begin{array}{l}8.9182 .03) \\
8.87 / 9.02 \\
9.22 / 8.63\left(^{*}\right) \\
8.93 / 8.96 / 8.50 \\
7.54 / 8.88 / 9.24\left(^{*}\right) \\
\end{array}$ & $\begin{array}{l}4.6183 .63) \\
4.77 / 4.16 \\
4.52 / 4.72 \\
4.60 / 4.80 / 5.13 \\
6.15 / 4.53 / 4.66 \\
\end{array}$ & $\begin{array}{l}8.59(2.49) \\
8.62 / 8.49 \\
8.85 / 8.35 \\
8.68 / 8.51 / 8.00 \\
7.54 / 8.58 / 8.83 \\
\end{array}$ \\
\hline 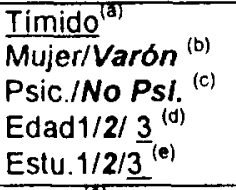 & $\begin{array}{l}7.23(2.79) \\
7.41 / 6.65 \\
7.84 / 6.72\left(^{*}\right) \\
7.53 / 6.22 / 7.88\left(^{\star}\right) \\
6.15 / 7.15 / 7.61 \\
\end{array}$ & \begin{tabular}{|l|}
$7.70(2.93)$ \\
$7.62 / 7.96$ \\
$8.06 / 7.40$ \\
$7.77 / 7.57 / 7.63$ \\
$5.69 / 7.78 / 7.90$ \\
\end{tabular} & $\begin{array}{l}8.88(1.99) \\
8.95 / 8.67 \\
9.34 / 8.48\left(^{*}\right) \\
8.86 / 8.98 / 8.63 \\
7.85 / 8.80 / 9.25\left(^{\star}\right) \\
\end{array}$ & $\begin{array}{l}5.31(3.47) \\
5.32 / 5.37 \\
5.75 / 4.99 \\
5.35 / 5.04 / 5.38 \\
4.38 / 5.57 / 5.02\end{array}$ & $\begin{array}{l}5.61(3.57) \\
5.90 / 4.67\left(^{*}\right) \\
5.98 / 5.31 \\
5.81 / 5.33 / 5.63 \\
4.77 / 5.73 / 5.55 \\
\end{array}$ \\
\hline 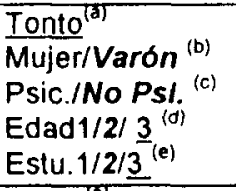 & $\begin{array}{l}6.76(3.44) \\
6.92 / 6.18 \\
7.23 / 6.35 \\
7.16 / 5.67 / 7.57\left(^{\star}\right) \\
6.69 / 6.65 / 7.12\end{array}$ & \begin{tabular}{|l|}
$6.69(3.31)$ \\
$6.64 / 7.37$ \\
$6.84 / 6.81$ \\
$6.76 / 7.18 / 6.88$ \\
$7.38 / 6.69 / 6.86$ \\
\end{tabular} & $\begin{array}{l}8.51(2.56) \\
8.47 / 8.61 \\
8.77 / 8.27 \\
8.53 / 8.63 / 8.00 \\
7.38 / 8.45 / 8.83 \\
\end{array}$ & $\begin{array}{l}2.56(3.15) \\
2.46 / 2.94 \\
2.33 / 2.80 \\
2.72 / 2.06 / 3.63 \\
2.85 / 2.81 / 2.02 \\
\end{array}$ & $\begin{array}{l}2.14(2.83) \\
2.25 / 1.82 \\
1.89 / 2.37 \\
2.27 / 1.65 / 3.00 \\
2.77 / 2.34 / 1.56 \\
\end{array}$ \\
\hline $\begin{array}{l}\text { Torpe }^{(a)} \\
\text { Mujer/Varon }^{(b)} \\
\text { Psic./No Psi. } \\
\text { Edad } 1 / 2 / 3^{(d)} \\
{\text { Estu. } 1 / 2 / 3^{(e)}}\end{array}$ & $\begin{array}{l}6.55(3.01) \\
6.74 / 5.90 \\
7.19 / 6.02\left(^{*}\right) \\
6.77 / 5.94 / 6.63 \\
6.62 / 6.36 / 6.95 \\
\end{array}$ & $\begin{array}{l}7.17(3.15) \\
7.06 / 7.49 \\
7.32 / 7.04 \\
7.11 / 7.67 / 6.38 \\
6.54 / 7.00 / 7.64 \\
\end{array}$ & $\begin{array}{l}8.96(1.90) \\
9.00 / 8.82 \\
9.22 / 8.73 \\
8.90 / 9.20 / 9.25 \\
8.62 / 8.84 / 9.29 \\
\end{array}$ & $\begin{array}{l}4.96(3.50) \\
5.01 / 4.86 \\
5.26 / 4.71 \\
5.05 / 4.78 / 4.38 \\
4.00 / 4.95 / 5.19 \\
\end{array}$ & \begin{tabular}{|l|}
$5.11(3.54)$ \\
$5.33 / 4.35$ \\
$4.46 / 4.78$ \\
$5.09 / 5.16 / 3.50$ \\
$3.08 / 5.41 / 4.91$ \\
\end{tabular} \\
\hline 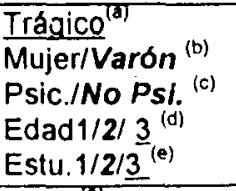 & $\begin{array}{l}5.10(3.21) \\
5.26 / 4.57 \\
5.74 / 4.57\left(^{\star}\right) \\
5.35 / 4.16 / 6.25\left(^{\star}\right) \\
5.23 / 4.88 / 5.53 \\
\end{array}$ & $\begin{array}{l}7.19(3.05) \\
7.15 / 7.45 \\
7.52 / 7.03 \\
7.36 / 7.13 / 6.25 \\
5.00 / 7.36 / 7.30\left({ }^{\star}\right)\end{array}$ & \begin{tabular}{|l}
$8.60(2.24)$ \\
$8.64 / 8.51$ \\
$8.99 / 8.27\left(^{\star}\right)$ \\
$8.58 / 8.71 / 9.00$ \\
$8.08 / 8.48 / 8.93$ \\
\end{tabular} & $\begin{array}{l}7.07(3.17) \\
7.46 / 5.85\left(^{\star}\right) \\
7.54 / 6.68\left(^{*}\right) \\
7.34 / 6.65 / 5.38 \\
6.85 / 7.12 / 7.17 \\
\end{array}$ & $\begin{array}{l}6.62(3.25) \\
6.70 / 6.37 \\
6.74 / 6.56 \\
6.99 / 5.41 / 7.25\left(^{*}\right) \\
6.77 / 6.77 / 6.38 \\
\end{array}$ \\
\hline $\begin{array}{l}\text { Triste }^{(\mathrm{d})} \\
\text { Mujer/Varón }^{(\mathrm{b})} \\
\text { Psic./No Psi. } \\
\text { Edad } 1 / 2 / \underline{3}^{\text {(d) }} \\
\text { Estu. } 1 / 2 / 3^{(\mathrm{e})}\end{array}$ & $\begin{array}{l}7.55(2.71) \\
7.74 / 6.96 \\
7.92 / 7.26 \\
7.73 / 6.94 / 8.13 \\
7.54 / 7.37 / 7.95 \\
\end{array}$ & \begin{tabular}{|l}
$8.16(2.71)$ \\
$8.18 / 8.08$ \\
$8.55 / 7.80\left(^{*}\right)$ \\
$8.21 / 7.94 / 9.13$ \\
$6.69 / 8.09 / 8.61$ \\
\end{tabular} & $\begin{array}{l}9.12(1.86) \\
9.14 / 9.04 \\
9.45 / 8.83\left({ }^{*}\right) \\
9.08 / 9.42 / 8.88 \\
8.23 / 9.05 / 9.45 \\
\end{array}$ & $\begin{array}{l}8.93(2.01) \\
9.13 / 8.31\left(^{\star}\right) \\
9.36 / 8.56\left(^{\star}\right) \\
8.97 / 8.96 / 8.00 \\
8.77 / 8.86 / 9.12 \\
\end{array}$ & $\begin{array}{l}.91(3.40) \\
3.88 / 4.04 \\
3.54 / 4.25 \\
3.70 / 4.67 / 3.50 \\
5.31 / 3.90 / 3.74 \\
\end{array}$ \\
\hline
\end{tabular}




\begin{tabular}{|c|c|c|c|c|c|}
\hline & Frec. de uso & Imaginación & Comprensión & Depresión & Ansiedad \\
\hline Turbado $^{\text {(a) }}$ & $3.50(2.99)$ & $5.52(3.28)$ & $7.94(2.45)$ & $5.63(3.39)$ & $5.99(3.25)$ \\
\hline Mujer/Varón ${ }^{(b)}$ & $3.40 / 3.78$ & $5.45 / 5.84$ & $8.04 / 7.67$ & $5.48 / 6.06$ & $6.18 / 5.33$ \\
\hline Psic./No Psi. ${ }^{(c)}$ & $3.90 / 3.17$ & $5.67 / 5.46$ & $8.32 / 7.61\left(^{*}\right)$ & $5.86 / 5.45$ & $6.51 / 5.50\left(^{*}\right)$ \\
\hline Edad1/2/ $\underline{3}^{(0)}$ & $3.57 / 3.08 / 4.50$ & $5.42 / 5.69 / 2.38$ & $7.77 / 8.57 / 7.75$ & $5.69 / 5.57 / 6.13$ & $6.11 / 5.67 / 5.13$ \\
\hline Estu. $1 / 2 / \underline{3}^{-(e)}$ & $3.92 / 3.26 / 3.97$ & $5.38 / 5.30 / 6.00$ & $7.23 / 7.69 / 8.61\left({ }^{*}\right)$ & $6.69 / 5.41 / 6.05$ & $6.23 / 5.72 / 6.50$ \\
\hline Vacilante $^{(2)}$ & $4.09(3.12)$ & $6.35(3.16)$ & $7.89(2.69)$ & $4.39(3.57)$ & $5.10(3.51)$ \\
\hline Mujer/Varónn ${ }^{(b)}$ & $4.01 / 4.37$ & $6.23 / 6.75$ & $7.84 / 8.04$ & $4.32 / 4.63$ & $5.08 / 5.16$ \\
\hline Psic./No Psi. (c) & $4.33 / 3.90\left(^{*}\right)$ & $6.38 / 6.37$ & $8.35 / 7.47\left(^{*}\right)$ & $5.21 / 3.71\left(^{*}\right)$ & $5.51 / 4.75$ \\
\hline $12 / 3^{(d)}$ & $1.27 / 3.75$ & $6.33 / 6.67 / 5.38$ & $7.71 / 8.33 / 8.63$ & $4.15 / 4.69 / 6.63$ & $5.36 / 4.59 / 3.13$ \\
\hline $2 / \underline{3}^{(e)}$ & $2.69 / 3.97 / \overline{4.58}$ & $5.92 / 6.25 / 6.66$ & $\left.6.77 / 7.64 / 8.61{ }^{*}\right)$ & $4.31 / 4.19 / \overline{4.97}$ & $3.31 / 5.31 / \overline{5.14}$ \\
\hline Vértigo $^{(2)}$ & $4.95(3.33)$ & $7.38(3.05)$ & $8.47(2.45)$ & $3.33(3.22)$ & $6.94(3.47)$ \\
\hline Mujer/Varón & $5.08 / 4.44$ & $7.32 / 7.55$ & $8.50 / 8.37$ & $3.25 / .3 .65$ & $6.95 / 7.02$ \\
\hline Psic./No Psi. & $5.37 / 4.57$ & $7.74 / 7.05$ & $8.86 / 8.12\left({ }^{*}\right)$ & $2.82 / 3.77\left(^{*}\right)$ & $7.47 / 6.53\left(^{*}\right)$ \\
\hline Edad 1/2/ $\underline{3}_{(\mathrm{e})}^{(\mathrm{d})}$ & $5.15 / 4.29 / 5.71$ & $7.47 / 7.35 / 7.13$ & $8.54 / 8.45 / 8.25$ & $3.27 / 3.42 / 5.00$ & $7.24 / 6.24 / 6.00$ \\
\hline Estu.1/2/3 $\underline{3}^{(e)}$ & $4.85 / 4.93 / 5.02$ & $6.62 / 7.52 / 7.09$ & $6.62 / 8.45 / 8.84\left({ }^{*}\right)$ & $5 8 \longdiv { 2 . 5 5 }$ & $5 . 3 7 / 7 . 0 8 \longdiv { 7 . 0 2 }$ \\
\hline Vicitima $^{(3)}$ & $5.22(3.07)$ & $7.12(2.98)$ & $8.87(1.90)$ & $6.79(3.45)$ & $5.71(3.56)$ \\
\hline Mujer/Varónn ${ }^{(b)}$ & $5.18 / 5.31$ & $7.11 / 7.25$ & $8.86 / 8.90$ & $6.78 / 6.84$ & $5.94 / 5.08$ \\
\hline Psic./No Psi. ${ }^{\text {(c) }}$ & $6.00 / 4.56\left(^{*}\right)$ & $7.27 / 7.07$ & $8.98 / 8.76$ & $6.78 / 6.80$ & $5.51 / 5.96$ \\
\hline $\operatorname{Edad} 1 / 2 / \underline{3}^{|0|}$ & $5.43 / 4.47 / 6.25$ & $7.23 / 7.16$ & $8.78 / 9.02 / 9.25$ & $6.76 / 7.16 / 7.00$ & $5.85 / 5.71 / 3.75$ \\
\hline Estu.1/2/3 ${ }^{(\mathrm{e})}$ & $5.31 / 5.11 / \overline{5.42}$ & $5.77 / 7.33 / 6.93$ & $8.62 / 8.81 / \overline{9.02}$ & $6.85 / 6.71 / \overline{7.02}$ & $6.77 / 5.88 / 5.00$ \\
\hline Violento $^{(J)}$ & $5.94(3.24)$ & $8.24(2.56)$ & $8.86(2.22)$ & $4.08(3.34)$ & $7.49(2.92)$ \\
\hline Mujer/Varónn ${ }^{\text {(b) }}$ & $5.97 / 5.78$ & $8.25 / 8.18$ & $8.97 / 8.50$ & $4.09 / 4.00$ & $7.64 / 7.12$ \\
\hline Psic./No Psi. ${ }^{\left({ }^{\prime}\right)}$ & $6.78 / 5.22\left(^{*}\right)$ & $8.77 / 7.76\left(^{*}\right)$ & $9.41 / 8.38\left({ }^{*}\right)$ & $3.61 / 4.47$ & $7.68 / 7.42$ \\
\hline Edad $1 / 2 / \underline{3}^{(d)}$ & $6.24 / 5.14 / 5.75$ & $8.21 / 8.27 / 8.88$ & $8.82 / 9.04 / 9.25$ & $3.93 / 4.82 / 3.63$ & $7.69 / 7.14 / 7.50$ \\
\hline Estu. $1 / 2 / \underline{3}^{-(e)}$ & $4.25 / 5.91 / \overline{6.34}$ & $7.42 / 8.15 / 8.53$ & $8.17 / 8.63 / \overline{9.49}\left({ }^{*}\right)$ & $5.08 / 4.16 / \overline{3.84}$ & $7.15 / 7.96 / 6.71\left(^{*}\right)$ \\
\hline
\end{tabular}

(a) Se indica la media para la muestra total. La desviación típica está indicada entre paréntesis

(b) Se indica las medias para las mujeres y los varones (en negrita).

(c) Se indica las medias para psicólogos y no sicólogos (en negrita). El asterisco (*) indica si existen diferencias significativas $(p<0.005)$.

(d) El grupo 1 corresponde a los sujetos entre 17 y 24 años; el grupo 2 (en negrita) a los sujetos entre 25 y 40 años; el grupo 3 (subrayado) a los sujetos entre 41 y 73 años. El asterisco (") indica si existen diferencias significativas $(p<0.005)$.

(e) El grupo 1 corresponde a los estudios elementales; el grupo 2 (en negrita) a los estudios medios: y el grupo 3 (subrayado) a los estudios superiores. El asterisco $\left(^{*}\right)$ indica si existen diferencias significativas $(p<0.005)$ 


\section{ANEXO 2: \\ LAS PALABRAS SEGÚN SU RELACIÓN CON ANSIEDAD VERSUS DEPRESIÓN}

\begin{tabular}{|c|c|c|c|c|}
\hline \multicolumn{2}{|c|}{$\begin{array}{l}\text { PALABRAS CON UNA MAYOR } \\
\text { RELACIÓN CON ANSIEDAD(*) }\end{array}$} & \multicolumn{2}{|c|}{$\begin{array}{l}\text { PALABRAS CON UNA MAYOR } \\
\text { RELACIÓN CON DEPRESIÓN(*) }\end{array}$} & $\begin{array}{l}\text { SIN DIFERENCIAS } \\
\text { ANSIEDAD-vs. } \\
\text { DEPRESIÓN } \\
\text { avergonzado }\end{array}$ \\
\hline $\begin{array}{l}\text { accidente } \\
\text { agitado } \\
\text { agonizante } \\
\text { alarmado } \\
\text { amenaza } \\
\text { amenazado } \\
\text { ansioso } \\
\text { aprensión } \\
\text { arriesgado } \\
\text { asustado } \\
\text { aterrorizado } \\
\text { dañino } \\
\text { defensivo } \\
\text { desconfiado } \\
\text { desesperado } \\
\text { dubitativo } \\
\text { embarazoso } \\
\text { emocionado } \\
\text { hipocondría } \\
\text { hostil } \\
\text { incómodo } \\
\text { inmaduro } \\
\text { inoportuno } \\
\text { inquieto } \\
\text { insomnio } \\
\text { intranquilo } \\
\text { irritable }\end{array}$ & $\begin{array}{l}\text { mareo } \\
\text { miedo } \\
\text { miedoso } \\
\text { molesto } \\
\text { nervioso } \\
\text { odioso } \\
\text { palpitación } \\
\text { pánico } \\
\text { peligro } \\
\text { perplejo } \\
\text { perseguido } \\
\text { preocupación } \\
\text { preocupado } \\
\text { problemático } \\
\text { rubor } \\
\text { sobresalto } \\
\text { sofoco } \\
\text { sudor } \\
\text { sudoroso } \\
\text { taquicardia } \\
\text { tembloroso } \\
\text { tensión } \\
\text { tenso } \\
\text { terror } \\
\text { vacilante } \\
\text { vértigo } \\
\text { violento }\end{array}$ & $\begin{array}{l}\text { achacoso } \\
\text { cansado } \\
\text { débil } \\
\text { deficiente } \\
\text { deprimido } \\
\text { desamparado } \\
\text { desanimado } \\
\text { desastre } \\
\text { descuidado } \\
\text { desdeñado } \\
\text { desdichado } \\
\text { desgracia } \\
\text { desinterés } \\
\text { despreciable } \\
\text { desprecio } \\
\text { destruido } \\
\text { dolorido } \\
\text { enfermizo } \\
\text { enfermo } \\
\text { entumecido } \\
\text { estúpido } \\
\text { fatiga } \\
\text { fracasado } \\
\text { fracaso } \\
\text { herido } \\
\text { humillación } \\
\text { ignorante }\end{array}$ & $\begin{array}{l}\text { imperfecto } \\
\text { inapetente } \\
\text { incapacidad } \\
\text { incapaz } \\
\text { incompetente } \\
\text { indefenso } \\
\text { inepto } \\
\text { inferior } \\
\text { insatisfecho } \\
\text { inútil } \\
\text { lento } \\
\text { llanto } \\
\text { lloroso } \\
\text { muerte } \\
\text { mutilado } \\
\text { patético } \\
\text { peor } \\
\text { pobre } \\
\text { rechazado } \\
\text { ridiculo } \\
\text { solitario } \\
\text { suicida } \\
\text { suicidio } \\
\text { tonto } \\
\text { triste } \\
\text { victima }\end{array}$ & $\begin{array}{l}\text { avergonzado } \\
\text { castigo } \\
\text { catástrofe } \\
\text { criticado } \\
\text { culpable } \\
\text { inadecuado } \\
\text { indeciso } \\
\text { inestabilidad } \\
\text { inseguro } \\
\text { mareado } \\
\text { molestia } \\
\text { ofendido } \\
\text { paralizado } \\
\text { simple } \\
\text { tímido } \\
\text { torpe } \\
\text { trágico } \\
\text { turbado }\end{array}$ \\
\hline
\end{tabular}

